Gut and Liver, Vol. 9, No. 3, May 2015, pp. 267-317

\title{
2014 KLCSG-NCC Korea Practice Guideline for the Management of Hepatocellular Carcinoma
}

\author{
Korean Liver Cancer Study Group (KLCSG) and National Cancer Center, Korea (NCC)
}

The guideline for the management of hepatocellular carcinoma (HCC) was first developed in 2003 and revised in 2009 by the Korean Liver Cancer Study Group and the National Cancer Center, Korea. Since then, many studies on HCC have been carried out in Korea and other countries. In particular, a substantial body of knowledge has been accumulated on diagnosis, staging, and treatment specific to Asian characteristics, especially Koreans, prompting the proposal of new strategies. Accordingly, the new guideline presented herein was developed on the basis of recent evidence and expert opinions. The primary targets of this guideline are patients with suspicious or newly diagnosed HCC. This guideline provides recommendations for the initial treatment of patients with newly diagnosed HCC. (Gut Liver 2015;9:267-317)

Key Words: Carcinoma, hepatocellular; Management; Guidelines

\section{INTRODUCTION}

\section{Introduction}

The guideline for the management of hepatocellular carcinoma (HCC) was first developed in 2003 and revised in 2009 by the Korean Liver Cancer Study Group (KLCSG) and the National Cancer Center (NCC), Korea. Since then, many studies on HCC have been carried out in Korea and other countries. In particular, a substantial body of knowledge has been accumulated on diagnosis, staging, and treatment specific to Asian characteristics, especially Koreans, prompting the proposal of new strategies. Accordingly, the new guideline presented herein was developed on the basis of recent evidence and expert opinions.

\section{Target population}

The primary targets of this guideline are patients with suspi- cious or newly diagnosed HCC. This guideline provides recommendations for the initial treatment of patients with newly diagnosed HCC. Moreover, this guideline includes some information about residual, progressed, and recurred tumors following initial treatment; however, it is not main topic of this guideline. Furthermore, preemptive antiviral therapies for underlying chronic hepatitis, management of cancer pain, and assessment of tumor response are also included to facilitate the use of the guideline in clinical practice.

\section{Intended users}

This revised guideline is intended to provide useful information and guidance for all Korean clinicians in charge of the diagnosis and treatment of HCC. It also provides trainee doctors and teachers practical information on the management of HCC.

\section{Developers and funding source}

The KLCSG proposed revising the KLCSG-NCC Korea Practice Guideline, and the NCC, Korea agreed. The KLCSG-NCC Korea Practice Guideline Revision Committee (KPGRC), which included hepatologists, oncologists, surgeons, radiologists, and radiation oncologists, was subsequently formed (Appendix 1). All required funding was provided by the NCC, Korea. Each member of the HCC-KPGRC collected and evaluated relevant evidence, and wrote the manuscript. Conflicts of interests of the HCC-KPGRC

\footnotetext{
*Annotation: These guidelines are organized opinions for which specialists reviewed current medical literature so that they may actually be used as references for clinical practice, research, and education about hepatocellular carcinoma. These guidelines are intended to be flexible in contrast to "standards of care," which are mandatory policies to be followed in every case. These guidelines were prepared by a joint collaboration of the Korean Liver Cancer Study Group (KLCSG) and the National Cancer Center (NCC), Korea. This may not be revised, changed, or assumed without prior consent from these two institutions.
}

Correspondence to: Korean Liver Cancer Study Group ${ }^{1}$ and National Cancer Center, Korea ${ }^{2}$

${ }^{1}$ 101-3304 Brown Stone Seoul, 464 Cheongpa-ro, Jung-gu, Seoul 100-717, Korea

E-mail: liver@klcsg.or.kr

${ }^{2} 323$ Ilsan-ro, Ilsan dong-gu, Goyang 410-769, Korea

http://www.ncc.re.kr

Received on November 25, 2014. Accepted on March 9, 2015.

pISSN 1976-2283 eISSN 2005-1212 http://dx.doi.org/10.5009/gnl14460

@ This is an Open Access article distributed under the terms of the Creative Commons Attribution Non-Commercial License (http://creativecommons.org/licenses/by-nc/3.0) which permits unrestricted non-commercial use, distribution, and reproduction in any medium, provided the original work is properly cited. 
members are summarized in Appendix 2.

\section{Evidence collection}

The HCC-KPGRC collected and evaluated the literature relevant to HCC management by searching in using MEDLINE (up to 2014) for updated revisions. Only English and Korean literature was searched. The search term "hepatocellular carcinoma" and other keywords related to clinical questions shown were used (Appendix 3); these clinical questions contained a wide range of key topics including epidemiology, prevention, diagnosis, staging, treatment, and assessment of tumor response.

\section{Levels of evidence and grades of recommendation}

Relevant literature was systematically reviewed. The evidence and recommendations were graded according to the Grading of Recommendations, Assessment, Development, and Evaluation (GRADE) system with minor modifications. ${ }^{1-4}$ The levels of evidence were assessed on the basis of the possibility of changes in the estimate of clinical impact by further research, and were categorized as high (A), moderate (B), or low (C). For example, A-level evidence is similar but not identical to that from one or more randomized controlled trials. If there is only a slight possibility of the level of evidence changing, because further randomized controlled trials are unlikely to be conducted, such evidence could be considered level A. According to the GRADE system, the grades of recommendation were classified as strong (1) or weak (2), collectively considering the level of evidence, quality, patient-centered outcomes, and socioeconomic aspects of each study. Therefore, each recommendation was graded on the basis of the level of evidence $(\mathrm{A}-\mathrm{C})$ and grades of recommendation (1 or 2) as follows: A1, A2, B1, B2, C1, or C2 (Table 1). This guideline avoided giving $\mathrm{C} 2$ grades.

\section{List of clinical questions}

The committee considered the following questions from four departments as key factors to be addressed in this guideline (Appendix 3). The committee reviewed the evidence and sug- gested recommendations through intra- and interdepartmental discussion.

\section{Manuscript review}

Drafts of the revised guideline were thoroughly reviewed at several intradepartmental, three interdepartmental, and four departmental head meetings. In addition to the contents, methodological validity was evaluated on the basis of the Appraisal of Guidelines for Research and Evaluation II (AGREE II) instrument., ${ }^{5,6}$ A revised draft of the manuscript was reviewed at an external review board meeting and an open symposium, and was modified further before publication. The external review board comprised eight specialists who are or were the head of the KLCSG or the Korean Association for the Study of the Liver (KASL). The final manuscript was endorsed by the board of executives of the KLCSG and the NCC, Korea.

\section{Guideline release}

The revised HCC guideline was presented at Liver Week 2014 (the Korean Association for the Study of the Liver-KLCSG-Korean Association of Hepato-biliary and Pancreas Surgery-Korean Liver Transplantation Society meeting) on June 14, 2014. The Korean edition is available at http://www.klcsg.or.kr or http:// ncc.re.kr.

\section{Plan for updates}

Updates will be planned when new major evidence regarding the diagnosis and/or treatment of HCC is accumulated.

\section{EPIDEMIOLOGY}

According to the central cancer registration statistics published in 2013, there were 218,017 cases of cancer in Korea in 2011. Among them, $7.6 \%(16,434)$ of all malignancies were primary liver cancer cases, ranking fifth in incidence. ${ }^{7}$ Regarding sex, 12,189 cases were in men and 4,274 cases were in women, making primary liver cancer the fourth and sixth most

Table 1. Grading of Recommendations, Assessment, Development, and Evaluation (GRADE)

\begin{tabular}{ll}
\hline Quality of evidence & Criteria \\
\hline High (A) & Further research is unlikely to change confidence in the estimate of the clinical effect. \\
Moderate (B) & Further research may change confidence in the estimate of the clinical effect. \\
Low (C) & Further research is very likely to impact confidence on the estimate of clinical effect. \\
\hline Strength of recommendation & Criteria \\
\hline Strong (1) & Factors influencing the strength of the recommendation included the quality of the evidence, presumed \\
& patient-important outcomes, and cost. \\
Weak (2) & Variability in preferences and values, or more uncertainty; recommendation is made with less certainty, \\
& higher cost or resource consumption.
\end{tabular}

Among the quality levels of evidence originally included in the GRADE system, we excluded "very low quality" (D) (i.e., the estimate of the effect is very uncertain) from this guideline for convenience. 
common cancer in men and women in Korea, respectively; the male:female ratio was 2.85:1. ${ }^{7}$ Regarding age, primary liver cancer occurred most frequently in patients in their 50s (28.6\%) followed by 60 s $(26.0 \%)$ and 70 s $(22.3 \%){ }^{7}$ The crude incidence rate, i.e., the number of newly occurring cancer patients during the observation period among the study population, was 32.9 (male, 48.6; female, 17.1) per 100,000 population. According to an annual report of the 2010 cancer registration statistics, the diagnostic code of HCC accounted for approximately 76.0\% among all primary liver cancers. According to the age-standardized incidence rate, compensated with the mid-year population in 2000, the incidences of primary liver cancer occurrence were 28.9 (male, 48.5; female, 12.6) per 100,000 population in 1999 and 22.8 (male, 36.7; female, 10.5) in 2011. This implies that the occurrence of primary liver cancer has decreased somewhat, showing an annual rate of change of $-1.8 \%$. However, the number of new cases of primary liver cancer in 1999 was 13,286 (male, 10,027; female, 3,259), and the sex ratio was similar to that of recent data, whereas the total number of cases is less than that of the recent data (Fig. 1). ${ }^{7,8}$ This may reflect the age structure of the Korean population, as the absolute number of liver cancer cases is increasing, whereas the age-standardized incidence is decreasing. ${ }^{9}$

Regarding regional distribution, in 1999, of HCC occurrence in Jeonnam, Gyeongbuk, and Gyeongnam was 28.9, 26.8, and 26.4 per 100,000 population, respectively. The occurrence of HCC was the lowest in Daejeon at 14.8 per 100,000 population; those in Gyeonggi and Seoul were 18.2 and 18.7 per 100,000

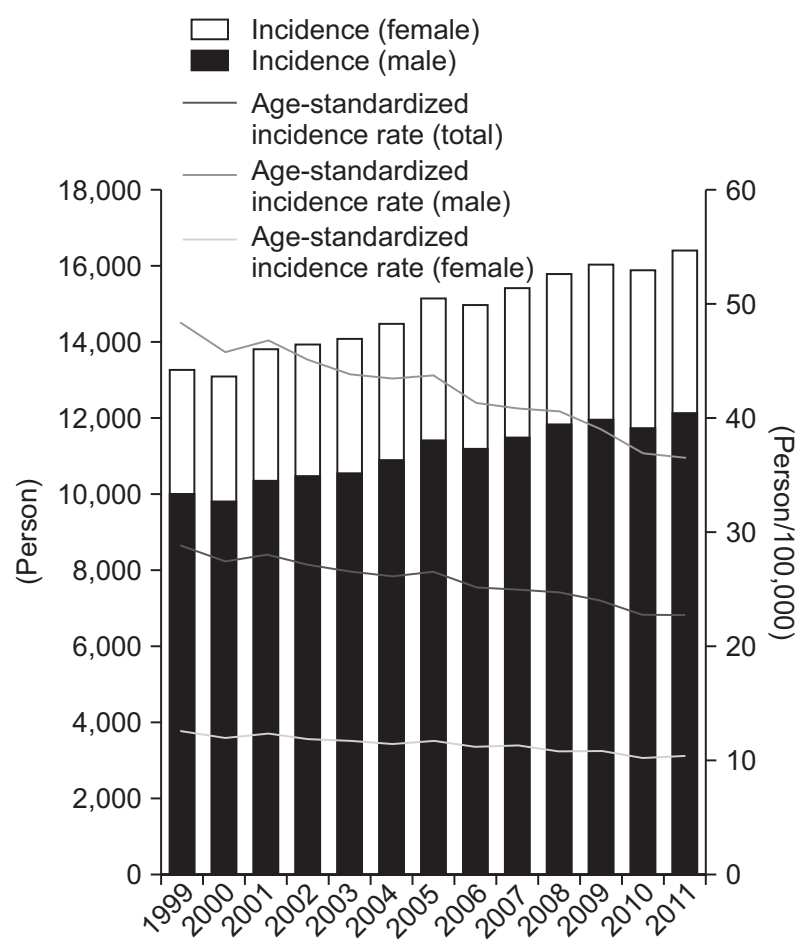

Fig. 1. Annual cases and incidence rate of hepatocellular carcinoma. ${ }^{8}$ population, respectively. In 2010, the regional incidences of HCC in Jeonnam, Jeju, Busan, Gyeongnam, Gyeongbuk, and Gangwon were 23.8, 20.8, 20.7, 20.4, 20.1, and 19 per 100,000 population, respectively. Regions of relative low incidence were Jeonbuk, Chungnam, Chungbuk, with 14.9, 15.1, and 15.6, respectively, as well as Seoul, Daegu, and Daejeon, each with 15.9 per 100,000 population. ${ }^{8,9}$

In 2011, the national primary liver cancer prevalence was 47,697 (age-standardized prevalence, 67.1 per 100,000 population), ranking sixth after thyroid, stomach, colon, breast, and lung cancers. Regarding the sex-specific prevalence, primary liver cancer was ranked at fourth in men (prevalence, 35,689; age-standardized prevalence, 107.2/100,000) and ninth in women (prevalence, 12,009; age-standardized prevalence, $31.3 / 100,000$ ); the male:female prevalence ratio was 2.97:1. Regarding 5-year prevalence, the age-standardized prevalence of primary liver cancer increased: 43.0 in 2007, 45.1 in 2008, 46.1 in 2009, 46.6 in 2010, and 47.9 per 100,000 population in 2011, respectively., ${ }^{7,8}$ The reason for the increasing prevalence of primary liver cancer despite its decreasing incidence is presumably because the survival rate of liver cancer patients is increasing. ${ }^{9}$

Liver cancer is the main cause of death of Korean men in their 50s. According to the National Statistics Office of Korea, 22.5 people (male, 33.7; female, 11.3) per 100,000 population die annually from liver cancer. While the most frequent cause of death in people after the age of 40 is malignancy, liver cancer is the top-ranked cause of death in people in their $40 \mathrm{~s}$ and $50 \mathrm{~s}^{10}$ According to a recent report of 10 principal cancers, shows that the 5-year cancer relative survival rates for liver cancer were 10.7\%, 13.2\%, 20.2\%, 27.3\%, and 28.6\% from 1993-1995, 1996-2000, 2001-2005, 2006-2010, and 2007-2011, respectively, showing consistent improvement (Fig. 2). However, the prognosis of liver cancer remains very poor compared to the $66.3 \%$ 5-year relative survival rates of all cancer patients from 2007 to 2011 in Korea. ${ }^{7,8}$ The prognosis of HCC is closely related to liver function. A report from Europe stated that a few patients excluded from curative treatment study (i.e., liver resection,

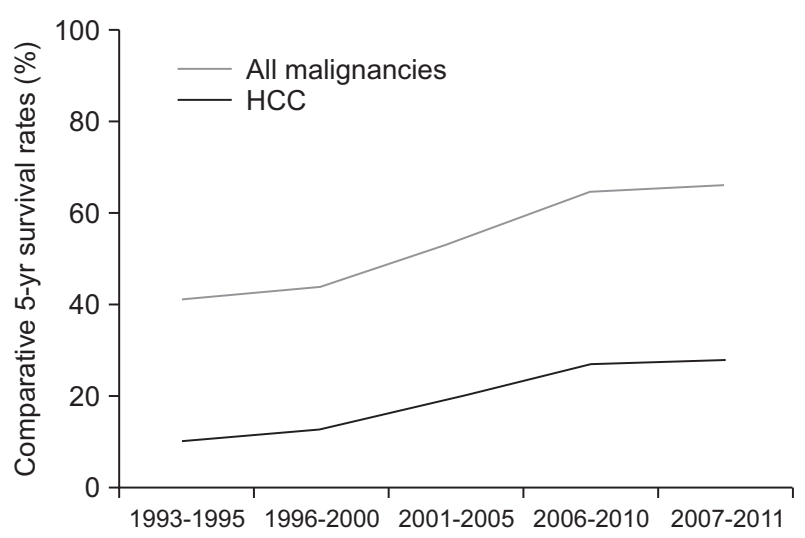

Fig. 2. Changes in the 5-year survival rates of overall cancers and hepatocellular carcinoma (HCC). ${ }^{8}$ 
liver transplantation [LT], and percutaneous ethanol injection) showed a 3-year survival rate of $28 \%$ without any treatment for HCC. ${ }^{11}$ However, this could be considered to be a result of selection bias. In Korea, according to the 2003-2005 randomized registration of HCC $(n=4,521)$, modified Union for International Cancer Control (mUICC) stages I, II, III, IVa, and IVb were 10.7\%, 33.4\%, 27.7\%, 10.3\%, and 7.9\%, respectively; the 3-/5-year survival rates in these stages were 62.3\%/52.0\%, 48.1\%/36.0\%, 22.3\%/15.5\%, 8.0\%/6.5\%, and 8.0\%/6.1\%, respectively. ${ }^{12}$ A recent single-center cohort study reports that 8.9\%, 29.6\%, 24.8\%, $23.1 \%$, and $13.6 \%$ of 1,972 patients with HCC from 2004 to 2009 were mUICC stage I, II, III, IVa, and IVb, respectively. ${ }^{13}$ The 5-year survival rates in stages I, II, III, IVa, and IVb were 71.1\%, 59.8\%, 25.0\%, 4.6\%, and 2.1\%, respectively, showing improvement in overall survival rates compared with the previous 2000-2003 cohort study. Improved survival rates in hepatitis B-related advanced HCC highlights the importance of antiviral therapy for hepatitis B-positive patients. ${ }^{13}$ The risk factors for HCC are relatively well known ${ }^{14,15}$ and include chronic hepatitis $\mathrm{B} / \mathrm{C}$, liver cirrhosis, alcoholic liver disease, obesity- and fatty liver disease-related diabetes mellitus, ${ }^{15,16}$ and aflatoxin. ${ }^{17}$ In Korea, one study reports that the underlying liver diseases of HCC patients included hepatitis B (72.3\%), hepatitis C (11.6\%), alcoholic liver disease in (10.4\%), and non-B non-C hepatitis (0.7\%). ${ }^{12}$ Another study reports that $74.6 \%$ of HCC patients were positive for hepatitis B virus (HBV), 9.3\% were positive for hepatitis C virus (HCV), 7.4\% were long-term alcohol abusers, and 8.7\% had unidentified causes (probably metabolic liver disease). ${ }^{13}$ HCC develops in $1 \%-4 \%$ of cirrhotic patients annually ${ }^{18}$ and eventually develops in approximately one-third of cirrhotic patients. ${ }^{19}$ In Korea, the number of carriers of hepatitis B decreased markedly because of hepatitis B vaccination and prevention services in vertical transmission. Although antiviral therapy for chronic hepatitis $\mathrm{B}$ and $\mathrm{C}$ is expected to further decrease the incidence of HCC, metabolic liver disease may increase as a cause of HCC in the future.

\section{PREVENTION}

The primary, secondary, and tertiary prevention of HCC development are defined as follows. Primary prevention of HCC can be achieved by universal vaccination against HBV infection, ${ }^{20}$ encouragement of healthy lifestyles preventing obesity and alcohol abuse, and controlling metabolic conditions. Secondary prevention is the prevention of HCC development in patients with chronic viral hepatitis through histologic improvement of inflammation and fibrosis by sustained suppression or eradication of hepatitis virus. Finally, tertiary prevention involves means to prevent recurrence in HCC patients after curative treatment.

The World Health Organization recommends vaccination against HBV for all newborns and high-risk groups. ${ }^{21}$ As peri- natal or early postnatal transmission is an important cause of chronic HBV infection worldwide, the first dose of hepatitis B vaccine should be administered as soon as possible after birth. Vaccination is also recommended for age-specific cohorts (i.e., young adolescents) and people with risk factors for acquiring $\mathrm{HBV}$ infection, including healthcare workers, travelers to areas where HBV-infection is prevalent, intravenous drug users, people with multiple sex partners, and adult patients who do not have hepatitis B surface antigen or surface antibody. However, there is currently no vaccination against HCV.

Antiviral therapies leading to sustained suppression of viral replication in chronic hepatitis B patients and inducing sustained virologic response in chronic hepatitis $\mathrm{C}$ patients are recommended because they prevent progression to cirrhosis and the development of HCC. Antiviral therapies should be administered according to the KASL guidelines for the management of chronic hepatitis B and C infections. ${ }^{22}$ Interferon and oral nucleos(t)ide analogues are now available for HBV treatment. Observational studies assessing the effect of interferon show a potential effect in the reduction of HCC incidence. ${ }^{23}$ Similarly, a randomized controlled trial assessing the effect of lamivudine shows a significant reduction in HCC incidence (32 months of follow-up; lamivudine vs placebo, 3.9\% vs 7.4\%; $\mathrm{p}=0.047$ ). ${ }^{24}$ Although HCC reduction after antiviral treatment remains controversial, ${ }^{25}$ a recent study shows significant reduction in HCC incidence in patients treated with entecavir ( 5 years of followup; entecavir vs control, 3.7\% vs $13.7 \%$; $\mathrm{p}<0.001){ }^{26}$

In chronic hepatitis $\mathrm{C}$ patients, whether interferon treatment reduces HCC risk (favors reducing; treated vs control, 4\% vs $38 \% ; p=0.002$ ) is controversial. ${ }^{27}$ However, others reported no significant difference in HCC incidence between treated and untreated controls. ${ }^{28}$ The results of a meta-analysis of 4,700 patients from 20 studies suggested the risk of HCC is reduced among interferon-treated patients (relative risk [RR], 0.43; 95\% confidence interval [CI], 0.33 to 0.56). ${ }^{29}$ Furthermore, the risk of HCC is reduced among patients with HCV who achieve a sustained virological response with antiviral therapy compared with nonsustained virological response (RR, 0.35; 95\% CI, 0.26 to 0.46$)$. However, once cirrhosis is established, there is no conclusive evidence that antiviral therapy can prevent or delay the occurrence of HCC. Previous studies including Hepatitis C Antiviral Long-term Treatment against Cirrhosis (HALT-C) ${ }^{30,31}$ and Evaluation of PegIntron in Control of Hepatitis C Cirrhosis (EPIC) studies ${ }^{32}$ show that maintenance therapy with pegylatedinterferon in cirrhotic patients does not significantly decrease the incidence of HCC; the study populations included in these trials were patients with nonresponse to prior interferon treatment. Therefore, additional studies are required to determine the potential preventive effect of monotherapy or combinations of new direct-acting antivirals with interferon in cirrhotic patients.

Regarding tertiary prevention for HCC, there is no conclusive evidence that antiviral therapy can prevent or delay HCC recur- 
rence after curative treatment. Yin et al. ${ }^{33}$ reported oral antiviral treatment against HBV significantly decreased HCC recurrence and HCC-related death after resection, with hazard ratios (HRs) of 0.48 (95\% CI, 0.32 to 0.70) and 0.26 (95\% CI, 0.14 to 0.50), respectively. In a study by Chen et al. ${ }^{34}$ that enrolled 268 patients (133 in the interferon $\alpha-2 b$ arm), the median recurrencefree survival in the interferon $\alpha-2 b$ and control arms were 42.2 (95\% CI, 28.1 to 87.1) and 48.6 (95\% CI, 25.5 to infinity) months, respectively $(\mathrm{p}=0.828)$. A meta-analysis including 551 patients from nine cohorts ${ }^{35}$ shows a significant difference in the incidence of HCC recurrence in favor of the antiviral treatment group (55\% vs 58\%; odds ratio [OR], 0.59; $\mathrm{p}=0.04$ ) after curative treatment of HBV related-HCC; the risk of HCC was reduced by $41 \%$ in the antiviral treatment group. There were also significant differences in favor of the antiviral treatment group with respect to liver-related mortality ( $0 \%$ vs $8 \%$; OR, 0.13; $\mathrm{p}=0.02$ ) and overall mortality (38\% vs 42\%; OR, 0.27; $\mathrm{p}<0.001$ ). Miao et al. ${ }^{36}$ performed a meta-analysis of the impact of postoperative antiviral treatment on tumor recurrence and survival of patients with chronic HBV or HCV infection-related HCC after curative therapy; 1,224 patients were included in their analysis. The estimated ORs for the 1-, 3-, and 5-year recurrence in HBVrelated HCC were $0.59,0.43$, and 0.21 , respectively.

In HCV-related HCC patients, Shiratori et al. ${ }^{37}$ report that the rates of second or third recurrence appeared to be lower in the interferon group than the untreated group. The 5- and 7-year survival rates of patients treated with interferon were of $68 \%$ and 53\%, respectively; those of untreated patients were $48 \%$ and 23\%, respectively. Mazzaferro et al. ${ }^{38}$ recently reported that while no treatment effect was apparent on early recurrence in HCV-positive patients, there was a significant benefit on late recurrence (HR, 0.3; $\mathrm{p}=0.04)$. According to Miao et al., ${ }^{36}$ the estimated ORs for the 1-, 3-, and 5-year recurrence in HCV-related HCC were significantly reduced to $0.52,0.23$, and 0.37 , respectively.

In addition to HBV-, HCV-, and alcohol-related chronic liver disease, associations between diabetes and obesity, with HCC were recently reported. ${ }^{39,40}$ Policies for preventing HCV/HBV transmission encourage lifestyles that prevent obesity and alcohol abuse as well as the control of metabolic conditions such as diabetes. Bravi et al. ${ }^{41}$ reported that coffee consumption reduced the RR of HCC compared to no coffee consumption: the summary RR was 0.72 for low consumption and 0.44 for high consumption. Their meta-analysis indicates the risk of HCC is reduced by $40 \%$ for any coffee consumption versus no consumption.

\section{Recommendations (Table 2)}

1. Hepatitis B vaccination is recommended for all newborns (A1) and high-risk individuals (i.e., HBsAg-negative and antiHBs-negative) (B1).

2. General preventive measures include the followings: pre- vention of $\mathrm{HBV} / \mathrm{HCV}$ transmission (A1), avoidance of alcohol abuse, and control of metabolic disorders such as obesity and diabetes (C1).

3. Antiviral therapy as secondary prevention against HCC should follow the KASL guidelines for the management of chronic hepatitis B/C (A1).

4. Antiviral therapy should be considered after curative treatment for chronic viral hepatitis-related HCC to reduce the risk of recurrence (B1).

\section{DIAGNOSIS}

The diagnosis of HCC is based on pathology or noninvasive criteria in high-risk groups. However, noninvasive criteria for HCC diagnosis vary greatly among guidelines. Most patients with HCC have definite risk factors such as HBV infection, HCV infection, and cirrhosis. Regular surveillance (i.e., abdominal ultrasound and serum $\alpha$-fetoprotein [AFP]) is warranted in these high-risk groups. ${ }^{42,43}$ The cost-effectiveness and survival benefit of patients with early HCC detected in surveillance programs have been documented in previous studies. ${ }^{44}$ Dynamic contrast-enhanced computed tomography (CT), dynamic contrast-enhanced magnetic resonance imaging (MRI), or MRI using hepatocyte-specific contrast agent (i.e., gadolinium ethoxybenzyl diethylenetriamine pentaacetic acid [Gd-EOB-DTPA]) are preferentially recommended if HCC is suspected in a surveillance program.

Serum AFP has been traditionally and widely used as a tumor marker of HCC. However, serum AFP level is normal in up to $35 \%$ of cases of small HCC and can be nonspecifically elevated in patients with active hepatitis or active hepatocyte regeneration. Although the glycosylated AFP/total AFP ratio (AFP-L3), des- $\gamma$-carboxy prothrombin or protein induced by vitamin $\mathrm{K}$ absence-II (PIVKA-II) have been suggested as new tumor markers for the diagnosis of HCC, none have demonstrated a definite role in the diagnosis of HCC. A recent Japanese guideline indicates the combined use of tumor markers (AFP $>200 \mathrm{ng} / \mathrm{mL}$, AFP-L3 >15\%, or PIVKA-II $>40 \mathrm{mAU} / \mathrm{mL}$ ) for the diagnosis of HCC. ${ }^{45}$ Several Korean retrospective studies also reported the clinical usefulness of these new tumor markers. ${ }^{46,47}$ However, further well-designed studies are warranted to confirm their roles in the diagnosis of HCC. If serum AFP level increases steadily over time, especially in hepatitis B patients with fully suppressed viral activity, the development of HCC should be suspected, and a detailed imaging study is strongly recommended. ${ }^{45,48,49}$

As the noninvasive diagnosis of HCC mostly depends on imaging studies, the sensitivity and specificity of imaging modalities are important for accurate diagnosis. Studies investigating the roles of imaging techniques in patients undergoing LT report that the diagnostic sensitivity of dynamic CT is $75.0 \%$, while those of dynamic MRI are $100 \%$ for HCC $\geq 2 \mathrm{~cm}$ in diameter 
Table 2. Summary of the Recommendations of the 2014 KLCSG-NCC Korea Practice Guidelines for the Management of Hepatocellular Carcinoma Topic Recommendation

Prevention

1. Hepatitis B vaccination is recommended for all newborns (A1) and for high-risk individuals (HBsAg-negative and anti-HBs-negative) (B1).

2. General preventive measures include the followings: prevention of HBV/HCV transmission (A1), avoidance of alcohol abuse, and control of metabolic disorders such as obesity and diabetes (C1).

3. Antiviral therapy as secondary prevention of HCC should follow the KASL guidelines for the management of chronic hepatitis B/C (A1).

4. Antiviral therapy should be considered after curative treatment for chronic viral hepatitis-related HCC in order to reduce the risk of recurrence (B1).

Diagnosis

1. HCC is diagnosed on the basis of either pathology or clinical criteria in case of the high-risk group (HBV/HCV positive or cirrhosis) (A1).

2. When HCC is suspected during surveillance in the high-risk group, dynamic contrast-enhanced CT/MRI or liverspecific contrast-enhanced MRI should be performed for diagnosis (B1).

3. In the high-risk group, HCC can be diagnosed for nodules $\geq 1 \mathrm{~cm}$ in diameter if one or two of the abovementioned imaging techniques show typical features of HCC (for the diagnosis of nodules $1-2 \mathrm{~cm}$ in diameter, two or more imaging modalities are required if a suboptimal imaging technique is used). Typical features of HCC include arterial phase enhancement with washout in the portal or delayed phase (B1).

4. Nodules $<1 \mathrm{~cm}$ in diameter can be diagnosed as HCC in high-risk patients when all of the following conditions are met: typical features of HCC in two or more of the abovementioned imaging modalities and continuously rising serum $\alpha$-fetoprotein with hepatitis activity under control (C1).

5. Pathological diagnosis should be considered when the clinical criteria are not met or typical features of HCC are not present. The presence of indeterminate nodules despite imaging workup or pathologic examination needs to be followed up with repeated imaging and serum tumor marker analysis (B1).

6. Limitation of radiation exposure in diagnosis and staging is not considered relevant in patients with HCC. CT is essential for diagnosis and follow-up in HCC patients (C1).

Staging

Surgical resection

Transplantation

Local ablation
1. This guideline adopts the modified Union for International Cancer Control stages as a primary staging system, with the Barcelona Clinic Liver Cancer staging system serving as a complementary system (B1).

1. Surgical resection is the first-line treatment for patients with intrahepatic single-nodular HCC and well-preserved liver function classified as Child-Pugh class A, without portal hypertension or hyperbilirubinemia (A1).

2. Limited resection can be selectively applied to HCC patients with liver function of Child-Pugh class A or superb B and with mild portal hypertension or mild hyperbilirubinemia (C1).

3. HCC resection can be considered in patients with three or fewer intrahepatic tumors without macrovascular invasion, if hepatic function is well preserved (C2).

4. Laparoscopy-assisted resection can be considered for HCC located in the lateral section of the left lobe or in the anterolateral segment of the right lobe (B2).

1. Deceased donor liver transplantation is the first-line treatment for patients with single-nodular $\mathrm{HCC}<5 \mathrm{~cm}$ in diameter or three or fewer nodules $\leq 3 \mathrm{~cm}$ in diameter (Milan criteria), which are not indicated for resection (A1).

2. Locoregional therapies (local ablation or TACE) are recommended if the timing of transplantation is not predictable (B1).

3. Downstaging (e.g., with TACE) can be considered for HCCs exceeding the criteria for transplantation (C2).

4. Living donor liver transplantation is an effective alternative to deceased donor transplantation (B1).

5. An expanded indication for transplantation beyond the Milan criteria can be considered in HCC cases without definitive vascular invasion or extrahepatic spread if other effective treatment options are inapplicable (C2).

6. Salvage transplantation can be indicated for recurrent HCC after resection according to the same criteria as for firstline transplantation (B1).

1. RFA provides survival comparable to that of resection in patients with single-nodular HCCs $\leq 3 \mathrm{~cm}$ in diameter (A2).

2. RFA is superior to PEIT in terms of anticancer effect and survival (A1). For HCCs $\leq 2 \mathrm{~cm}$ in diameter, PEIT can be considered if RFA is unfeasible, because the outcomes of both modalities are similar (A2).

3. Survival outcome can be improved by combining TACE and RFA compared to RFA alone in patients with tumors 3-5 $\mathrm{cm}$ in diameter if resection is unfeasible (A2). 
Table 2. Continued

\begin{tabular}{|c|c|}
\hline pic & Recommendation \\
\hline $\begin{array}{l}\text { Transarterial } \\
\text { chemoembolization } \\
\text { and other transarterial } \\
\text { treatments }\end{array}$ & $\begin{array}{l}\text { 1. TACE is recommended for patients with good performance status without major vascular invasion or extrahepatic } \\
\text { spread who are ineligible for surgical resection, liver transplantation, RFA, or PEIT (A1). } \\
\text { 2. TACE should be performed through tumor-feeding vessels using selective/superselective techniques to maximize an- } \\
\text { titumor activity and minimize hepatic damage (B1). } \\
\text { 3. Chemoembolization using drug-eluting beads results in less systemic adverse events and has similar therapeutic ef- } \\
\text { ficacy compared with conventional TACE (B2). } \\
\text { 4. In case of portal vein invasion, TACE can be considered for patients with localized tumor and well-preserved liver } \\
\text { function (B2). }\end{array}$ \\
\hline $\begin{array}{l}\text { External-beam } \\
\text { radiation therapy }\end{array}$ & $\begin{array}{l}\text { 1. EBRT can be performed in HCC patients if liver functions are Child-Pugh class A or superb B and the irradiated total } \\
\text { liver volume receiving } \geq 30 \mathrm{~Gy} \text { is } \leq 60 \% \text { (B1). } \\
\text { 2. EBRT can be considered for HCC patients ineligible for surgical resection, liver transplantation, RFA, PEI, or TACE (C1). } \\
\text { 3. EBRT can be considered for HCC patients who exhibit incomplete response to TACE when the dose-volume criteria } \\
\text { in Recommendation } 1 \text { are met (B2). } \\
\text { 4. EBRT can be considered for HCC patients with portal vein invasion when the dose-volume criteria in Recommenda- } \\
\text { tion } 1 \text { are met (C1). } \\
\text { 5. EBRT is performed to alleviate symptoms caused by primary HCC or its metastases (B1). }\end{array}$ \\
\hline System & $\begin{array}{l}\text { 1. Sorafenib is indicated for HCC patients with very well-preserved liver function (Child-Pugh class A), good per- } \\
\text { formance status, and regional lymph node or extrahepatic spread or for patients with tumor progression on other } \\
\text { therapies (A1). } \\
\text { 2. Sorafenib is recommended for HCC patients with very well-preserved liver function (Child-Pugh class A), good per- } \\
\text { formance status, and vascular invasion (A2). } \\
\text { 3. Sorafenib is considered for HCC patients with liver function Child-Pugh class superb B and good performance status } \\
\text { if the above conditions } 1 \text { and } 2 \text { are satisfied (B1). } \\
\text { 4. Cytotoxic chemotherapy can be considered for HCC patients with advanced tumors who have with well-preserved } \\
\text { liver function and, with good performance status, in whom sorafenib therapy has failed (C1). } \\
\text { 5. Adjuvant TACE, sorafenib, or cytotoxic chemotherapy are not recommended for HCC patients treated with curative } \\
\text { resection (B1). }\end{array}$ \\
\hline $\begin{array}{l}\text { Preemptive antiviral } \\
\text { therapy }\end{array}$ & $\begin{array}{l}\text { 1. Patients should be tested for the HBsAg before starting cytotoxic chemotherapy or immunosuppressive therapy (A1). } \\
\text { 2. Preemptive antiviral therapy is recommended for HBV carriers undergoing cytotoxic chemotherapy to prevent re- } \\
\text { activation (A1). Preemptive antiviral therapy is considered for HBV-infected patients receiving TACE (B1), hepatic } \\
\text { arterial infusion chemotherapy (C1), surgical resection (C1), or EBRT (C1) to prevent reactivation. } \\
\text { 3. Antiviral treatment for HBV reactivation should follow the recommendations of the current KASL guidelines (A1). }\end{array}$ \\
\hline $\begin{array}{l}\text { Drug treatment for } \\
\text { cancer pain in HCC }\end{array}$ & $\begin{array}{l}\text { 1. Careful consideration is required for pain management with medication in patients with HCC and underlying liver } \\
\text { disease. The dosage and dosing intervals of analgesics should be determined on the basis of liver functions (C1). } \\
\text { 2. In patients with HCC and chronic liver disease, the dosage of acetaminophen should be lowered (C1) and NSAIDs } \\
\text { should be used with caution (B1). } \\
\text { 3. In patients with HCC and chronic liver disease, opioid analgesics and their dosage should be selected carefully on } \\
\text { the basis of drug metabolism and liver function (C1). }\end{array}$ \\
\hline $\begin{array}{l}\text { Assessment of tumor } \\
\text { response and } \\
\text { posttreatment } \\
\text { follow-up }\end{array}$ & $\begin{array}{l}\text { 1. Assessment of tumor response should follow both the RECIST and modified RECIST criteria (B1). } \\
\text { 2. Patients with complete response after treatment should be followed up with imaging studies (i.e., dynamic contrast- } \\
\text { enhanced CT/MRI or liver-specific contrast-enhanced MRI) and serum tumor markers every 2-6 months in the first } \\
2 \text { years; thereafter, patients should be followed by regular checkups at individualized intervals (B1). }\end{array}$ \\
\hline
\end{tabular}

HBsAg, hepatitis B surface antigen; HBV, hepatitis B virus; HCV, hepatitis C virus; HCC, hepatocellular carcinoma; KASL, Korean Association for the Study of the Liver; CT, computed tomography; MRI, magnetic resonance imaging; TACE, transarterial chemoembolization; RFA, radiofrequency ablation; PEIT, percutaneous ethanol injection therapy; EBRT, external-beam radiation therapy; NSAIDs, nonsteroidal anti-inflammatory drugs; RECIST, Response Evaluation Criteria in Solid Tumors.

but only 52\% for HCC $<2 \mathrm{~cm}$ in diameter. ${ }^{50-52}$ The overall sensitivities of ultrasound, dynamic CT, and dynamic MRI for the noninvasive diagnosis of HCC are 61\%-67\%, 68\%-91\%, and $81 \%-100 \%$, respectively. ${ }^{53-55}$

If liver nodules are found during surveillance in high-risk groups, dynamic CT, dynamic MRI, or MRI using hepatocytespecific contrast agent should be performed. If the typical hallmark of HCC (i.e., hypervascularity in the arterial phase with washout in the portal or delayed phases) is identified by using the imaging techniques mentioned above, a nodule $\geq 1 \mathrm{~cm}$ in 
diameter can be diagnosed as HCC. Low, medium, and high signal intensity in the hepatocyte phase, T2-weighted image, and diffusion-weighted image, respectively, have been suggested as additional findings to support the diagnosis of HCC by MRI using a hepatocyte-specific contrast agent. ${ }^{56,57}$ However, further studies are required to confirm evidence on the role of these findings. The role of angiography in the diagnosis of HCC is quite limited owing to rapid advances in other noninvasive imaging techniques. Although contrast-enhanced ultrasonography has been introduced to characterize and localize small liver nodules (especially for radiofrequency ablation), its roles in the diagnosis and staging of HCC remain quite limited. ${ }^{58}$ Positron emission tomography-CT (PET-CT) is not recommended as a primary diagnostic imaging method, because its diagnostic accuracy is relatively low, especially in patients with small HCC. ${ }^{59}$

The accuracy of noninvasive diagnostic criteria largely depends on the size of the nodules. As the size of the nodules increases, their differentiation becomes poorer. ${ }^{60,61}$ As an HCC lesion grows, it begins to exhibit the typical hallmark of HCC, i.e., hypervascularity in the arterial phase with washout in the portal or delayed phases due to the gradual growth of tumor arteries and regression of portal flow. ${ }^{55,61}$ Nowadays, small nodules $<1$ $\mathrm{cm}$ in diameter showing the typical hallmark of HCC are being detected more frequently because of recent advances in imaging technologies. Many guidelines from Asia allow the diagnosis of HCC on the basis of these small nodules. ${ }^{45,48,62}$

The pathologic diagnosis of HCC requires an adequate specimen through biopsy. However, biopsy is not always feasible because of the location of the target lesion and potential risk of complications such as bleeding and tumor seeding, especially in cirrhotic patients. The diagnostic sensitivity of fine-needle aspiration cytology, fine-needle aspiration biopsy, and core needle biopsy are reported to be $67 \%$ to $93 \%$ but much lower in patients with small HCC $<2 \mathrm{~cm}$ or targeting problems. ${ }^{63,64}$ As the risk of tumor seeding due to needle biopsy is reported to be $0.6 \%$ to $5.1 \%$, the need for biopsy for patients with HCC curable by surgical resection is being challenged. ${ }^{65,66}$ In addition, the false negativity of biopsy is reported to be approximately $33 \%{ }^{64}$

The diagnosis of HCC for liver nodules detected by surveil- lance is based on noninvasive criteria or pathology (Table 3, Fig. 3). Noninvasive criteria can only be applied to high-risk groups (i.e., HBV/HCV infection and liver cirrhosis) and are based on imaging including dynamic CT, dynamic MRI, and MRI using a hepatocyte-specific contrast agent. Diagnosis should be based on the identification of the typical hallmark of HCC (i.e., hypervascularity in the arterial phase and washout in the portal or delayed phase) for liver nodules $\geq 1 \mathrm{~cm}$ in diameter. ${ }^{45,62,67}$ One or more imaging techniques are usually recommended for noninvasive diagnosis for these nodules; however, one or more imaging techniques are required in optimal settings (Appendices 5 and 6), whereas two or more are recommended in suboptimal settings for nodules $1-2 \mathrm{~cm}$ in diameter. ${ }^{58}$ In addition, stricter criteria are warranted for the diagnosis of HCC in cases of nodules $<1 \mathrm{~cm}$. Diagnosis should be based on the combination of the identification of the typical hallmark of HCC in two or more imaging modalities and increased serum AFP with an increasing trend over time for liver nodules $<1 \mathrm{~cm}$ in patients with suppressed hepatitis activity. ${ }^{57}$ Biopsy should be considered for atypical nodules not meeting the noninvasive criteria. Any changes in the size or characteristics of nodules or serum tumor markers should be monitored if noninvasive or pathologic diagnosis is unfeasible for liver nodules in high-risk patients.

\section{Diagnostic X-ray radiation exposure dose and risk of dy- namic CT in HCC patients}

A study of low-dose radiation in atomic bomb survivors indicates a significant increase in cancer risk even from acute 10-50 $\mathrm{mSv}$ radiation exposure ${ }^{68}$ In addition, studies of occupational radiation exposure suggest protracted 50-100 mSv exposure can increase cancer risk in humans. ${ }^{69-71}$ The International Commission on Radiological Protection (ICRP) reports that the cancer risk after radiation exposure exhibits a linear-nonthreshold dose-response relationship. ${ }^{72,73}$ However, there is no report on direct diagnostic X-ray radiation exposure-related cancer risk. The dose of radiation exposure of 4-phase liver dynamic CT is approximately 20-30 mSv. According to BEIR VII phase 2 trial by the Committee to Assess Heath Risks from Exposure to Low Levels of Ionizing Radiation, the additional lifetime attribut-

Table 3. Diagnosis of Hepatocellular Carcinoma

\footnotetext{
1. Noninvasive diagnosis: in high-risk groups (i.e., HBV/HCV infection, liver cirrhosis)

1) For liver nodules $1 \mathrm{~cm}$ found by surveillance:

If the typical hallmark of $\mathrm{HCC}^{*}$ is identified on one or more (two or more ${ }^{\dagger}$ ) imaging techniques ${ }^{\ddagger}$

2) For nodules $<1 \mathrm{~cm}$ found by surveillance:

If the typical hallmark of $\mathrm{HCC}^{*}$ is identified in two or more imaging techniques ${ }^{\ddagger}$ and increased serum AFP with an increasing trend over time is noted in patients with suppressed hepatitis activity
}

2. Pathologic diagnosis

HBV, hepatitis B virus; HCV, hepatitis C virus; HCC, hepatocellular carcinoma; AFP, $\alpha$-fetoprotein.

${ }^{*}$ Hypervascularity in the arterial phase and washout in the portal or delayed phase; ${ }^{\dagger}$ For $1-2-\mathrm{cm}$ nodules, the diagnosis should be based on the identification of the typical hallmark of HCC in one or more imaging techniques in optimal settings (Appendices 5 and 6) and in two or more imaging techniques in suboptimal settings; ${ }^{\ddagger}$ Dynamic computed tomography, dynamic magnetic resonance imaging, gadolinium-ethoxybenzyldiethylenetriamine pentaacetic acid (Gd-EOB-DTPA)-enhanced magnetic resonance imaging magnetic resonance imaging. 
able solid cancer and leukemia incidence and mortality rates are $0.148 \%$ and $0.09 \%$, respectively, in 50-year-old men with $25 \mathrm{mSv}$ X-ray radiation exposure after a 4-phase liver dynamic CT. ${ }^{74,75}$ The ICRP 2007 recommendations are as follows: "The limitation of the dose to the individual patient is not recommended because it may, by reducing the effectiveness of the patient's diagnosis or treatment, do more harm than good. The emphasis is then on the justification of the medical procedures and on the optimization of protection." ${ }^{76}$ Thus, considering the abovementioned factors, any limitation of the cumulative radiation dose from CT for the diagnosis and follow-up evaluation of HCC is invalid.

\section{Recommendations (Table 2)}

1. HCC is diagnosed on the basis of either pathology or clinical criteria in case of the high-risk group (HBV/HCV positive or cirrhosis) (A1).

2. When HCC is suspected during surveillance in the highrisk group, dynamic contrast-enhanced CT/MRI or liver-specific contrast-enhanced MRI should be performed for diagnosis (B1).

3. In the high-risk group, HCC can be diagnosed for nodules $\geq 1 \mathrm{~cm}$ in diameter if one or two of the above-mentioned imaging techniques show typical features of HCC (for the diagnosis of nodules $1-2 \mathrm{~cm}$ in diameter, two or more imaging modalities are required if a suboptimal imaging technique is used). Typical features of HCC include arterial phase enhancement with washout in the portal or delayed phase (B1).

4. Nodules $<1 \mathrm{~cm}$ in diameter can be diagnosed as HCC in high-risk patients when all of the following conditions are met: typical features of HCC in two or more of the above-mentioned imaging modalities and continuously rising serum AFP with hepatitis activity under control (C1).

5. Pathological diagnosis should be considered when the clinical criteria are not met or typical features of HCC are not present. The presence of indeterminate nodules despite imaging workups or pathologic examination needs to be followed up with repeated imaging and serum tumor marker analysis (B1).

6. Limitation of radiation exposure in diagnosis and staging is not considered relevant in patients with HCC. CT is essential for diagnosis and follow-up in HCC patients (C1).

\section{STAGING}

Cancer staging plays a pivotal role in predicting prognosis as well as selecting the therapy to maximize survival potential. It also facilitates exchange of information and trial design. The prediction of prognosis in HCC patients is complex, because underlying liver function also affects prognosis. ${ }^{77,78}$ Although several staging systems for patients with HCC have been devised, there is no worldwide consensus. ${ }^{79}$

The American Joint Committee on Cancer (AJCC) has led a collaborative effort with the International Union for Cancer Control (UICC) to maintain a cancer staging system since 1959. This system classifies the extent of disease mostly on the basis of anatomic information on the extent of the primary tumor, regional lymph nodes, and distant metastases (i.e., TNM) and has been modified repeatedly. The Korean Liver Cancer Study Group and The Korea National Cancer Center have adopted the fifth version of the mUICC staging system as a primary staging system for HCC in 2003 (Table 4). ${ }^{80}$ Thus, the continuous use of this staging system since then may facilitate consistency in the analyses of registry data. ${ }^{62}$ The mUICC staging system appears more advantageous for estimating the prognosis of small HCC because it sets the size criterion to $2 \mathrm{~cm}$ unlike the AJCC/ UICC, which used a criterion of $5 \mathrm{~cm} .{ }^{81}$ However, the fifth ver-

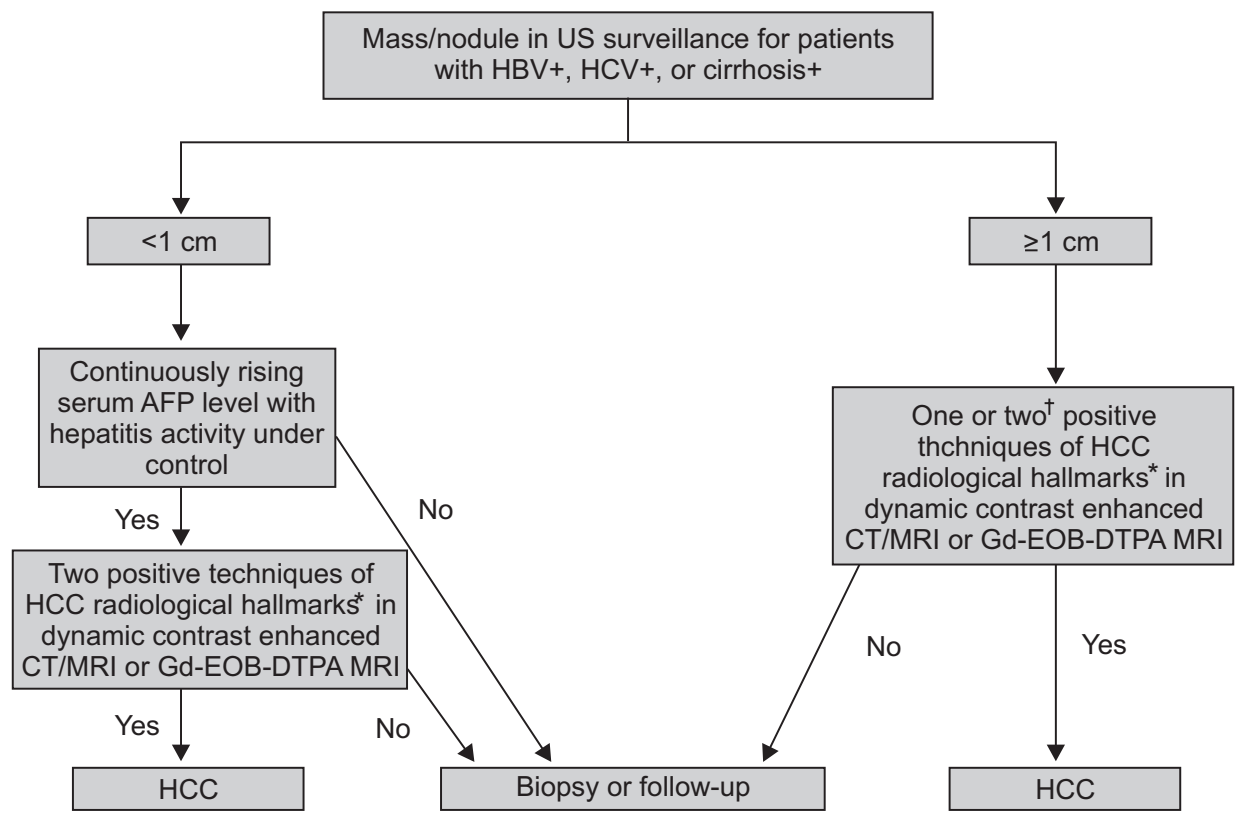

Fig. 3. Diagnostic algorithm. US, ultrasonography; HBV, hepatitis $B$ virus; $\mathrm{HCV}$, hepatitis $\mathrm{C}$ virus; AFP, $\alpha$-fetoprotein; HCC, hepatocellular carcinoma; CT, computed tomography; MRI, magnetic resonance imaging; Gd-EOB-DTPA, gadolinium ethoxybenzyl diethylenetriamine pentaacetic acid. *HCC radiological hallmarks include arterial phase enhancement with washout in the portal or delayed phase; ${ }^{\dagger}$ For the diagnosis of nodules $1-2 \mathrm{~cm}$ in diameter, two or more imaging modalities are required if a suboptimal imaging technique is used. 
Table 4. Modified Union for International Cancer Control Staging System*

\begin{tabular}{lccc}
\hline Stage & T & N & M \\
\hline I & T1 & N0 & M0 \\
II & T2 & N0 & M0 \\
III & T3 & N0 & M0 \\
IV A & T4 & N0 & M0 \\
& T1, T2, T3, T4 & N1 & M0 \\
IV B & T1, T2, T3, T4 & N0, N1 & M1
\end{tabular}

*Adapted from the Liver Cancer Study Group of Japan. ${ }^{80,81}$

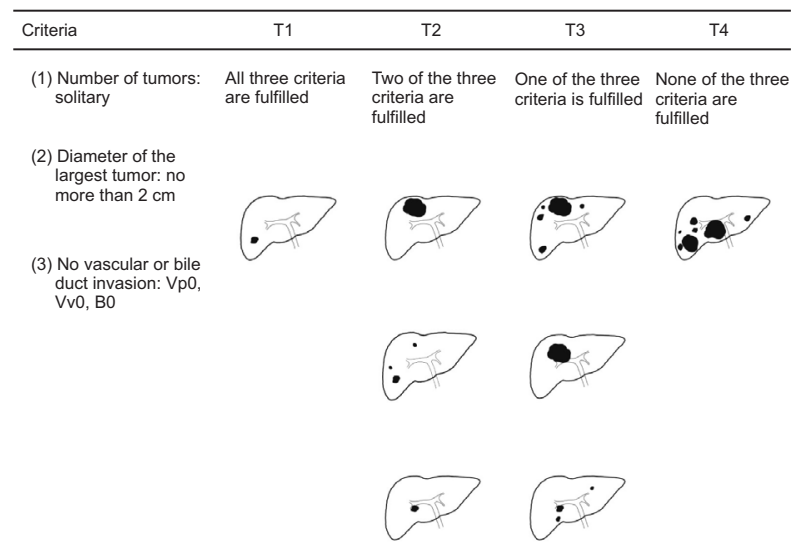

sion of the mUICC staging system has limitations, particular its lack of extensive validation and different criteria compared with those of the current seventh AJCC/UICC TNM staging system. In addition to dynamic CT or MR imaging of the primary liver tumor, chest CT, bone, and PET-CT scans may be required to stage HCC. The risk of distant metastasis is low for patients with early-stage HCC; ${ }^{82}$ therefore, tests for the evaluation of extrahepatic metastasis should be carefully selected. The direct or indirect evaluation of portal hypertension is also required, especially for patients who are being considered for surgical resection.

The Barcelona Clinic Liver Cancer (BCLC) staging system includes factors for tumor stage, degree of liver function, and performance status of the patient. It suggests the most recommendable treatment modality for each stage and is being endorsed by the American Association for the Study of Liver Diseases (AASLD), the European Association for the Study of the Liver (EASL), and the European Organisation for Research and Treatment of Cancer (EORTC) ${ }^{58,83}$ However, the use of the BCLC staging system is limited because it contains a subjective component (i.e., performance status), crude evaluation of liver function (i.e., Child-Pugh class), and unduly simplified recommendations for treatment modality.

\section{Recommendations (Table 2)}

1. This guideline adopted the mUICC stages as a primary staging system, with the BCLC staging system serving as a complementary system (B1).

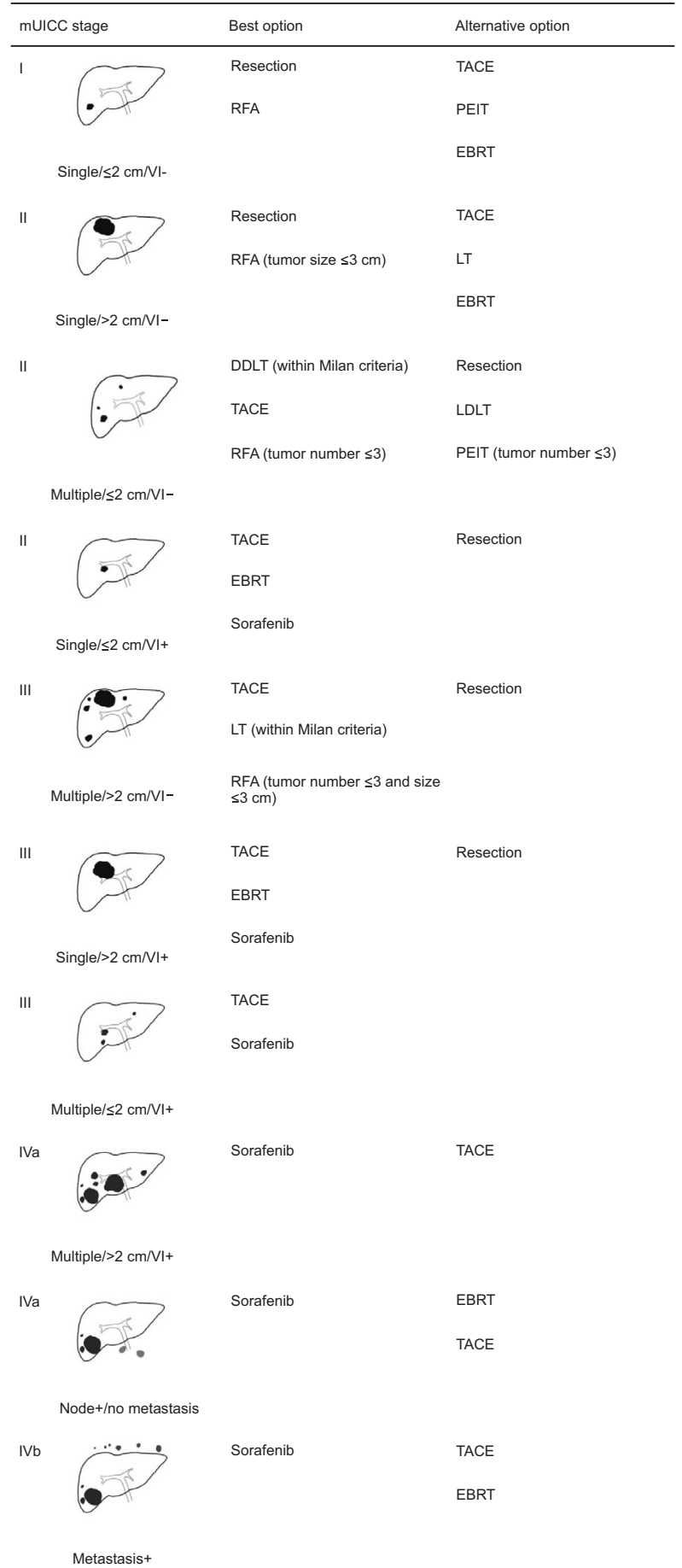

Fig. 4. First-line treatment according to the 2014 KLCSG-NCC Korea Practice Guidelines for patients with HCC, Child-Pugh class A, no portal hypertension, and ECOG performance status 0-1.

KLCSG, Korean Liver Cancer Study Group; NCC, National Cancer Center; HCC, hepatocellular carcinoma; ECOG, Eastern Cooperative Oncology Group; mUICC, modified Union for International Cancer Control; RFA, radiofrequency ablation; TACE, transarterial chemoembolization; PEIT, percutaneous ethanol injection therapy; EBRT, external-beam radiation therapy; LT, liver transplantation; DDLT, deceased donor LT; LDLT, living donor liver transplantation; VI, vascular or bile duct invasion. 


\section{TREATMENT OVERVIEW}

The ultimate goal of treatment for HCC is improvement of patient survival. This requires multidisciplinary treatment planning including hepatology, oncology, surgery, diagnostic and interventional radiology, radiation oncology, and pathology. Therapies should be selected on the basis of strong evidence such as meta-analyses including randomized controlled trials (RCTs), prospective controlled studies, and prospective largescale cohort studies, all of which have survival as an endpoint. However, RCTs regarding HCC are limited. Therefore, currently available evidence should be interpreted cautiously. For balanced multidisciplinary treatment planning, objective evaluation is necessary in clinical practice.

The recommendations in this guideline were derived from the current best available evidence. Prerequisites for the application of these recommendations include equipment, trained personnel, and financial resources; considering the varying levels of these prerequisites among facilities, best and alternative options are proposed herein (Fig. 4). As the treatment options presented herein do not encompass all possible situations, specific treatments should be selected on the basis of tumor state, liver function, cirrhotic complications, and performance status. Recommendations for specific treatments are made on the basis of evidence and expert opinions, and are described in detail elsewhere in this guideline.

This overview summarizes treatment options for patients in various mIUCC stages with well-preserved hepatic function (i.e., Child-Pugh class A) and good performance status, without any

Table 5. Child-Pugh Classification

\begin{tabular}{lccc}
\hline & 1 & 2 & 3 \\
\hline Albumin, g/dL & $>3.5$ & $2.8-3.5$ & $<2.8$ \\
Bilirubin, mg/dL & $<2.0$ & $2.0-3.0$ & $>3.0$ \\
Prothrombin time prolonged, sec & $0-4$ & $4-6$ & $>6$ \\
Ascites & None & Slight & Moderate \\
Encephalopathy, grade & None & $1-2$ & $3-4$ \\
\hline
\end{tabular}

Class $\mathrm{A} \leq 6$ points, Class $\mathrm{B}=7-9$ points, Class $\mathrm{C} \geq 10$ points. complications of portal hypertension. In addition, this guideline mainly includes initial treatment options for HCC at first diagnosis, without covering re-treatments for residual tumors or recurrence after initial treatment.

\section{HEPATIC RESECTION}

Hepatic resection is not only a primary treatment option for HCC unaccompanied by liver cirrhosis, ${ }^{84}$ but should also be preferentially considered even for HCC with cirrhosis if the liver function is expected to be able to tolerate surgery. ${ }^{85,86}$ The results of hepatic resection for HCC have markedly improved thanks to recent advances in preoperative tests and surgical skills as well as accumulation of experience in postoperative management. ${ }^{87}$ Recent studies show that postoperative mortality after HCC resection is less than 1\%-3\%. In addition, the 5-year overall and disease-free survival rates are 46\%-56\% and 23\%-32\%, respectively. ${ }^{88-90}$

Child-Pugh classification is conventionally used to preoperatively assess the safety of hepatic resection (Table 5). ${ }^{91}$ Hepatic resection is commonly performed in patients with Child-Pugh class A with Eastern Cooperative Oncology Group (ECOG) performance status 0-2 (Table 6). However, Child-Pugh classification is an insufficient preoperative indicator of operability, because many patients can remain in Child-Pugh class A despite advanced cirrhosis. ${ }^{92,93}$ Therefore, the indocyanine green 15-minute retention rate (ICG-R15), which was suggested in Japan, is evaluated at many Korean institutions as a preoperative test for the prediction of residual liver function. ${ }^{94}$ Although major hepatic resection is recommended only for patients with ICG-R15 $\leq 10 \%$, some authors recently reported safely performing right hemihepatectomy even in patients with an ICG-R15 of $14 \% .{ }^{95}$ On the other hand, portal hypertension and serum bilirubin level have been suggested to be important indicators for the assessment of resectability in Europe and the USA. Portal hypertension is defined as a hepatic venous pressure gradient $\geq 10$ mm Hg. ${ }^{96}$ Esophageal varix and thrombocytopenia $<100,000 /$ $\mathrm{mm}^{3}$ accompanied by splenomegaly are also indicators of portal hypertension, and thrombocytopenia is considered the most clinically useful. ${ }^{58}$ The posthepatectomy complication rate is

Table 6. Eastern Cooperative Oncology Group Performance Status*

\begin{tabular}{cl}
\hline Grade & \multicolumn{1}{c}{ ECOG } \\
\hline 0 & Fully active, able to carry out all pre-disease performance without restriction \\
1 & Restricted in physically strenuous activity, but ambulatory and able to carry out work of a light or sedentary nature, e.g., light house \\
& work, office work \\
2 & Ambulatory and capable of all self-care, but unable to carry out any work activities. Up and about more than 50\% of waking hours \\
3 & Capable of only limited self-care, confined to bed or chair more than 50\% of waking hours \\
4 & Completely disabled. Cannot perform any self-care. Totally confined to bed or chair \\
5 & Dead
\end{tabular}

ECOG, Eastern Cooperative Oncology Group.

*Oken MM, et al. Toxicity and response criteria of the Eastern Cooperative Oncology Group. Am J Clin Oncol 1982;5:649-655. 
high and the long-term prognosis is poor in patients with portal hypertension. ${ }^{96-98}$ However, some recent studies show comparable outcomes can be achieved even in patients with portal hypertension. ${ }^{99-101}$ Therefore, hepatic resection can also be considered the primary treatment option in patients with mild portal hypertension if LT is unavailable. Minor hepatic resection less than hemihepatectomy should be considered in patients with mild portal hypertension, because resection volume is closely associated with the risk of postoperative hepatic insufficiency.

Assessment of future liver volume or remnant liver volume after resection is as important as the hepatic reservoir function test in order to predict postoperative hepatic insufficiency. HCC is accompanied by chronic liver disease in $80 \%$ of cases. Although $70 \%-80 \%$ of the liver's volume can be resected for normal livers, much less resection volume is allowed for diseased livers. There are few studies about the safe remnant liver volume in patients with cirrhosis. Nevertheless, a remnant liver volume $\geq 40 \%$ is generally recommended in cirrhotic patients for safety. ${ }^{102}$ Several noninvasive tests to measure the severity of hepatic fibrosis have been developed. Among them, transient elastography was recently reported to be effective for predicting postoperative hepatic failure. ${ }^{103-105}$ In the near future, transient elastography is expected to play an important role in the preoperative assessment of hepatic functional reservoir.

Dynamic contrast-enhanced CT is the basic test utilized as a preoperative radiologic study to assess the possibility of resection. MRI using a hepatic cell-specific contrast medium is superior to CT for HCC detection, especially for small HCCs $<1$ $\mathrm{cm}^{106,107}$ Nevertheless, more studies are required to validate the value of MRI as the basic radiologic study for assessing resectability and formulating resection plans. Further postoperative examinations may be necessary to find extrahepatic metastases in patients with advanced HCC. ${ }^{18} \mathrm{~F}$-fluoro-2-deoxyglucose $\left({ }^{18} \mathrm{~F}\right.$ FDG) PET-CT may be effective for investigating extrahepatic metastasis, although its sensitivity is very low for intrahepatic lesions. ${ }^{59,108}$ In addition, chest CT and bone scan may be helpful. ${ }^{109}$

Transarterial chemoembolization (TACE), performed before hepatic resection for the purpose of improving postoperative prognosis, is not recommended. ${ }^{110,111}$ Some reports assert that portal vein embolization before extensive hepatic resection might induce compensatory hypertrophy of the residual liver and reduce the risk of surgery, especially in cases with liver cirrhosis; nonetheless, these are largely debatable. ${ }^{12,113}$

One reason why hepatic resection has recently become safer is the reduction in the amount of intraoperative hemorrhage, thus minimizing the amount of blood transfusion required. Blood transfusion compromises anticancer immunologic mechanisms and increases postoperative recurrence. A recent meta-analysis reports that intraoperative transfusion increases complication rates and reduces overall and disease-free survival rates after resection in HCC patients. ${ }^{114}$ Owing to selective hepatic blood flow occlusion, maintaining low central venous pressure, and precise transection of the hepatic parenchyma, the recent rate of transfusion in hepatic resection is $\leq 10 \% .{ }^{115}$ Although some reports suggest anatomical resection may be superior to nonanatomical resection by securing the resection margin and removing micro-metastases, ${ }^{116}$ further confirmation is required, because the results are inconsistent. ${ }^{117}$ Surgery guaranteeing a tumorfree resection margin is absolutely critical for improving longterm prognosis. One prospective randomized trial shows that a resection margin $>2 \mathrm{~cm}$ leads to better outcomes after HCC resection. ${ }^{118}$ Meanwhile, another study reports that the resection margin width is not important so long as a tumor-negative margin is guaranteed. ${ }^{119}$ Therefore, although sufficient margin from the tumor and anatomical resection are recommended during HCC resection if possible, considering patient safety is more important, because excessive hepatic resection can be fatal in patients with cirrhosis. ${ }^{116,120,121}$

Laparoscopic hepatic resection has advanced rapidly, and its indications have been expanded. Many studies show that the efficacy and safety of laparoscopic hepatic resection is comparable to those of open hepatic resection for small HCCs located in the left lateral section or on the surface of the right liver. ${ }^{122}$ Although laparoscopic major hepatic resection is increasingly being performed as well, it is currently limited to only experienced surgeons. Accordingly, its efficacy and safety should be evaluated further. ${ }^{123}$ Robotic hepatic resection has recently been tried in very select cases, and comparative studies between robotic hepatic resection and open or laparoscopic hepatic resection are expected. ${ }^{124}$

The best prognosis after hepatic resection is generally expected in cases involving 1 or 2 small tumors. Larger tumors are associated with a high incidence of vascular invasion result in poor prognosis even after resection. However, a recent study shows that approximately one-third of large HCCs $\geq 10 \mathrm{~cm}$ have no vascular invasion and favorable results after resection in those cases. Therefore, the resectability for HCC should not be decided upon according to tumor size. ${ }^{125-127}$ Recent advances in surgical techniques and improvements in patient management have enabled hepatic resection in elderly patients with comparable short- and long-term outcomes. Nevertheless, major hepatic resection should be considered with caution, because hepatic regeneration power gradually decreases with age. ${ }^{128-130}$

Although some authors reported that one-stage hepatic resection was an effective method for ruptured HCC in patients with good liver function, ${ }^{131}$ first hemostasis by TACE and subsequent elective surgery after accurate assessment of hepatic reservoir function would be safer and more effective in hemodynamically unstable patients. ${ }^{132,133}$ However, ruptured cases have poorer long-term results than unruptured HCC cases. ${ }^{134}$ Hepatic resection is generally contraindicated in cases with evident tumor invasion to major hepatic or portal veins. In patients with less hepatic fibrosis or those with a well-differentiated HCC of 
low Edmondson-Steiner grade, the 5-year survival rate after resection of HCC with major vascular invasion is reported to be $\geq 20 \%$. ${ }^{135}$ Nevertheless, surgical resection would be at least more effective than medical treatment. ${ }^{136}$ According to a Korean multicenter study, the 5-year survival rate after resection of HCC with bile duct invasion was satisfactory at $32 \% .{ }^{137}$ Hence, surgical resection can be considered even for HCC with major vascular invasion or bile duct invasion in select cases.

The hanging maneuver is frequently used during hepatic resection. However, there is no report about the effect of the hanging maneuver on survival or recurrence after HCC resection. Nevertheless, the hanging maneuver can shorten operative time and reduce the amount of bleeding. ${ }^{138}$ The anterior approach, which is often used for the resection of large tumors, is associated with less bleeding, a lower transfusion rate, and better survival in one prospective study. ${ }^{139}$ However, its pathologic advantages require further evaluation.

The 5-year recurrence rate after hepatic resection of HCC ranges from $58 \%$ to $81 \%$, and $80 \%$ to $95 \%$ of postoperative recurrences are intrahepatic. ${ }^{140}$ Intrahepatic recurrences are divided into intrahepatic metastasis and de novo HCC by multicentric carcinogenesis. The two recurrence entities can be differentiated by means of genomic hybridization, DNA fingerprinting, DNA microarray, or HBV integration pattern. ${ }^{141}$ However, no clinical definition of either entity has been established. In general, late recurrence more than 2 years after primary resection is considered de novo HCC. ${ }^{142}$ Risk factors associated with recurrence after resection are classified as tumor-related factors and underlying disease-related factors. Tumor-related risk factors, which are usually related to early recurrence, include tumor size and number, microvascular invasion, poor tumor differentiation, high serum AFP and PIVKA-II levels, and positivity on ${ }^{18} \mathrm{~F}$ FDG PET. Meanwhile, underlying disease-related risk factors, which influence late recurrence, include cirrhosis, high serum HBV DNA level, and active hepatitis. ${ }^{59,142-148}$ Nevertheless, no association between risk factors and recurrence time is evident in many cases, because this time-dependent classification does not actually reflect the tumor-pathologic mechanism of HCC recurrence.

Imaging modalities such as CT and MRI as well as serum tumor markers are recommended surveillance tools during followup. Serum AFP, a traditional tumor marker of HCC, is not only useful for the diagnosis of HCC, but is also effective for checking for recurrence after resection. PIVKA-II is another HCC marker with increasing utility for diagnosis, follow-up, and prognostication of HCC. ${ }^{147,149}$

The 5-year survival rate of patients who undergo re-resection of intrahepatic recurrence after initial surgery ranges from 37\% to $70 \% .^{140,150-152}$ Because the result of re-resection is excellent in cases with a long interval between initial surgery and tumor recurrence, re-resection can be recommended particularly for patients with late intrahepatic recurrence 1-2 years after initial resection as long as vascular invasion of the tumor is not evident and liver function is tolerable to re-operation. In addition, salvage transplantation could result in an excellent disease-free survival rate $>60 \%$ if the conditions of the patient and recurrent tumors are suitable for transplantation. ${ }^{153,154}$ Extrahepatic recurrence develops in 15\%-37\% of cases after HCC resection, most frequently in the lungs followed by the abdominal cavity and bones. ${ }^{155}$ Metastatectomy can also be considered when the liver function can tolerate surgery and intrahepatic HCCs have been clearly treated or are controllable. ${ }^{156,157}$

\section{Recommendations (Table 2)}

1. Surgical resection is the first-line treatment for patients with intrahepatic single-nodular HCC and well-preserved liver function of Child-Pugh class A without portal hypertension or hyperbilirubinemia (A1).

2. Limited resection can be selectively applied to HCC patients with liver function Child-Pugh class A or superb B and with mild portal hypertension or mild hyperbilirubinemia (C1).

3. HCC Resection can be considered in patients with three or fewer intrahepatic tumors without macrovascular invasion if hepatic function is well preserved (C2).

4. Laparoscopy-assisted resection can be considered for HCC located in the lateral section of the left lobe or anterolateral segment of the right lobe (B2).

\section{LIVER TRANSPLANTATION}

LT is the first choice of treatment for patients with single tumors $\leq 5 \mathrm{~cm}$ or those with small multinodular tumors ( $\leq 3$ nodules $\leq 3 \mathrm{~cm}$ ) and advanced liver dysfunction. LT involves complete removal of a diseased liver including HCC and replacement with another liver. It is theoretically the ideal treatment method. The application of broad selection criteria in the early history of LT resulted in very poor outcomes, with a 5-year survival less than $40 \%$; there was even a time when LT was relatively contraindicated. ${ }^{158,159}$ However, this resulted in the identification of the best candidates, and subsequent studies with highly specific groups of patients report a 5-year disease-free survival rate of 74\%. ${ }^{160,161}$ The Milan Group in Italy reports excellent results after LT, showing that patients with the following characteristics have 4-year survival and disease-free survival rates of 75\% and 83\%, respectively: (1) no extrahepatic metastasis or vascular infiltration in radiologic study before LT; (2) a single nodule $\leq 5 \mathrm{~cm}$; (3) $\leq 3$ nodules in cases of multiple nodules, with each nodule $\leq 3 \mathrm{~cm}$. Accordingly, they suggest those criteria for LT in patients with HCC. ${ }^{162}$ Since then, the Milan criteria have widely been applied for LT in patients with HCC. A recent systematic review of 90 studies including a total of 17,780 patients over 15 years identified the Milan criteria as an independent prognostic factor after LT. The overall 5-year survival rate of patients meeting the Milan criteria (65\%-78\%) is similar to that of non- 
HCC patients according to European and American transplant registries. ${ }^{163-165}$

Recent advances in imaging technologies have enabled the noninvasive diagnosis of HCC with higher accuracy. However, small lesions that could not be detected by imaging studies when the Milan criteria were established can often be seen on imaging studies using current technologies, causing confusion as to whether a patient meets the Milan criteria or not. A recent meta-analysis including 22,392 patients concludes that the size of the largest tumor and total diameter of nodules are the best predictors of outcome but that there is insufficient evidence regarding the effect of nodule number on the outcome of LT. ${ }^{166}$ Sugimachi et al. ${ }^{167}$ also report poor diagnostic accuracy of radiologic imaging for small $(<1 \mathrm{~cm})$ HCCs and their small effect on prognosis after LT. Therefore, lesions $\leq 10 \mathrm{~mm}$ or showing atypical imaging features should not be considered when deciding upon LT.

Before LT, patients with HCC undergo tests for staging in addition to general whole-body examination. Dynamic contrastenhanced CT or MRI is performed as a radiologic assessment of the liver itself. Extrahepatic staging should include chest CT, and abdominal and pelvic CT or MRI. ${ }^{168}$ Brain imaging and bone scintigraphy can also be performed. Moreover, ${ }^{18} \mathrm{~F}-\mathrm{FDG}$ PET-CT can help clarify the biologic characteristics of HCC, because PET-positive tumors exhibit unfavorable histological features more frequently (e.g., high cellular dedifferentiation and microvascular invasion), resulting in worse recurrence-free survival after LT. ${ }^{169,170}$ Although there is neither a specific study nor consensus on the optimal timing or modality of evaluation of wait-listed patients to ensure they continue to meet the acceptability criteria for LT, re-evaluation with a 3-month interval is commonly performed with dynamic CT or MRI and AFP measurement. ${ }^{171}$

\section{Deceased donor liver transplantation}

Deceased liver donors are always in shortage. Accordingly, many patients are waiting for LT at any given time. Patients with HCC must undergo a long waiting period from registration to LT. The American United Network for Organ Sharing (UNOS) introduced the Model for End-stage Liver Disease (MELD) scoring system in order to decide on the priority order for LT. Patients with HCC involving a single nodule between 2 and $5 \mathrm{~cm}$ or multinodular tumors ( $\leq 3$ nodules $\leq 3 \mathrm{~cm}$ ) are given the priority MELD score of 22 points as well as 10\% additional points for every 3 months waiting for LT; thus, similar risks of dropout from the waiting-list can be expected between HCC patients and non-HCC candidates. ${ }^{172,173}$ Meanwhile, Korean the National Organ Transplantation Management Center operates the Korean Network for Organ Sharing (KONOS) grading system. ${ }^{174}$ In this system, no additional points are given to patients with HCC. The provision in the KONOS grading system regarding patients with HCC specifies that cases with a Child-Turcott-Pugh score $\geq 7$ points and simultaneously meeting the Milan criteria belong to KONOS grade $2 \mathrm{~B}$. Patients with KONOS grade $2 \mathrm{~B}$ are pushed back in the priority order, and will not be able to undergo LT in a short time. ${ }^{175}$ According to a recent multicenter study in Korea ( $\mathrm{n}=1,101$; mean follow-up, 349 days), 23.5\% of waitlisted patients with HCC dropped out. The most frequent reason for dropping out was aggravation of liver function (46.7\%), followed by aggravation of HCC (36.3\%). Aggravation of HCC was observed in $44.8 \%$ (241/538) of patients while on the waiting list; $14.2 \%$ and $48.1 \%$ of aggravations occurred within 1 year and between 1 and 2 years after listing, respectively. ${ }^{176}$ The overall survival of patients with HCC was significantly worse than that of patients without HCC. The impact of HCC on the LT waiting list drop-out rate was significant only in patients with a MELD score $<20 .{ }^{176,177}$

If the cutoff for the definition of a cure is a 5-year survival rate of 50\% after LT in patients with HCC, the indications for LT could be expanded beyond the Milan criteria. ${ }^{178,179}$ Some studies chose a 5-year survival rate cutoff of 70\%, because it is similar to the rate expected for patients undergoing LT for noncancerous conditions and liver grafts from deceased donors. ${ }^{180-182}$ The University of California, San Francisco (UCSF) Group reports a 5-year survival rate of 75\% for patients meeting the following criteria: (1) a single tumor $\leq 6.5 \mathrm{~cm}$; (2) $<3$ nodules in multiple HCC cases, with the longest diameter $<4.5 \mathrm{~cm}$ and the sum of diameters $<8 \mathrm{~cm} .{ }^{183}$ In addition, the "up-to-seven criteria," (i.e., the number 7 as the sum of the largest tumor diameter plus the number of tumors) have also been suggested; the 5-year survival rate of patients meeting these criteria is $71.2 \%$. ${ }^{184}$ Microvascular invasion in pretransplant biopsy, tumor volume, and AFP levels are also considered in the expanded indications for LT. $^{185,186}$

\section{Bridging therapy}

The actuarial probability of dropping due to tumor progression while waiting for LT for 1 year is reported to range from $15 \%$ to $30 \%$. ${ }^{183}$ Locoregional therapies are reported to reduce the dropout rate to $0 \%-25 \% .{ }^{173,187}$ TACE or radiofrequency ablation (RFA) can be performed to prevent tumor progression. ${ }^{188-190}$ Markov-based cost-effectiveness analysis indicates benefits for neo-adjuvant treatments when waiting times exceed 6 months. ${ }^{188}$ AFP increasing $>15 \mu \mathrm{g} / \mathrm{L} /$ mo while waiting for LT is the most relevant preoperative prognostic factor for low overall survival and disease-free survival. ${ }^{185}$

The effects of neoadjuvant treatments on survival after LT are even more difficult to assess. Many studies report survival rates similar to those of untreated individuals; ${ }^{191-194}$ however, the major limitation of these studies is shorter waiting times ( $<6$ months) for LT. ${ }^{191,192}$ The response to bridging therapy significantly affects both tumor recurrence and overall survival rate. This result suggests that HCC candidates should be prioritized with respect to their response to bridging therapy ${ }^{195}$ that 
improved overall survival after LT is expected. ${ }^{196}$ The Organ Procurement and Transplantation Network/Scientific Registry of Transplant Recipients data regarding LT for HCC show a higher 3-year post-LT survival rate in patients who received ablative procedures before LT than those who did not regardless of preLT treatment modality. ${ }^{197}$ Radiation as a bridge to LT for advanced HCC is feasible, and well-selected patients are expected to achieve improved overall survival if HCC responds. ${ }^{198-200}$

\section{Downstaging}

Regarding downstaging, there are no randomized controlled trials, large case-control studies, or large well-designed cohort studies in which patients were treated consistently and properly followed up. Some prospective studies suggest downstaging according to the Milan or UCSF criteria as a result of locoregional therapies achieves 5-year survival outcomes similar to those within the Milan or UCSF criteria. ${ }^{190,201}$ However, it is unclear if downstaging therapies yield measurable anticancer effects. Downstaging of HCC with TACE may be possible in 24\%-63\% of cases. ${ }^{178,196,202,203}$ Downstaging is more effective in cases in which tumor size is $<7 \mathrm{~cm}$ or there are fewer than 3 tumors, ${ }^{203}$ but there is no clear upper limit for the eligibility of downstaging. ${ }^{204}$ Downstaging is possible with RFA or hepatic resection, but the efficacy of both remains inconclusive. ${ }^{187}$ Transarterial radioembolization (TARE) using ${ }^{90} \mathrm{Y}$ appears to outperform TACE for downstaging HCC from UNOS T3 to T2, and downstaged patients show a statistically insignificant trend toward improved overall survival after LT. ${ }^{205}$

\section{Living donor liver transplantation}

Patients with HCC in Korea have a very low probability of receiving deceased donor liver transplantation (DDLT) before tumor progression. ${ }^{206}$ According to the KONOS regulation for registration and allocation in Korea, LT recipient candidates with HCC cannot gain higher priority on the waiting list. ${ }^{174}$ These findings suggest DDLT is not a feasible treatment modality for patients with HCC in Korea. Therefore, living donor liver transplantation (LDLT) from a healthy donor has emerged as an alternative to DDLT as a treatment modality for HCC. In fact, a significant proportion of the LT recipients with HCC received transplantations from live donors in Korea. The proportion of adult LDLT recipients with HCC has recently increased to 30\%$40 \%$ in Korea, suggesting LDLT is now one of the main treatment modalities for HCC. ${ }^{207}$

The outcome of LDLT versus DDLT for patients with HCC is controversial. A meta-analysis of 633 LDLTs and 1,232 DDLTs ${ }^{208}$ indicates LDLT is an acceptable option that does not compromise survival rates. However, the disease-free survival rate is worse after LDLT than DDLT. The higher recurrence observed after LDLT is likely due to differences in tumor characteristics, pretransplant HCC management, and waiting time. ${ }^{209-211}$ The recurrence rate with respect to stage is higher in recipients whose transplants were accelerated (i.e., "fast-tracked") by performing LDLT, especially in the era in which patients with HCC are disadvantaged by the allocation algorithm. ${ }^{210}$ Cost-effectiveness studies suggest LDLT can be offered to patients with HCC if the waiting list exceeds 7 months. ${ }^{211}$ Some authors recommend an observation period, e.g., 3 months prior to LT, in order to avoid transplantation in cases with potentially aggressive tumors. ${ }^{210}$ However, these propositions require further investigation. Furthermore, future studies must have better study design and reporting in order to accurately describe the observed difference in disease-free survival due to inappropriate study design or biological risk specifically associated with LDLT. ${ }^{208}$

Several eligibility criteria besides the Milan criteria for LDLTs have been adopted by many high-volume LDLT centers. At Asan Medical Center, patients with $\leq 6$ HCCs $\leq 5 \mathrm{~cm}$ and without gross vascular invasion are considered eligible for LT; such patients have a 5-year survival rate of $81.6 \%$ at this center. ${ }^{212}$ At Seoul Catholic Medical Center, LDLT is considered the preferred therapeutic option in patients with an AFP level $<100 \mathrm{ng} / \mathrm{mL}$ and a tumor diameter $<5 \mathrm{~cm}$. The 5 -year disease-free survival and overall survival rates after LDLT in all patients with HCC are $80.9 \%$ and $76.4 \%$, respectively. ${ }^{213}$ At Seoul National University Hospital, the 3-year survival rate is reported to be $86.2 \%$ if vascular invasion was absent in preoperative radiological studies and preoperative AFP was $<400 \mathrm{ng} / \mathrm{mL}^{214}$ At Samsung Medical Center, patient selection according to tumor size $<5 \mathrm{~cm}$ and AFP $<400 \mathrm{ng} / \mathrm{mL}$ without limitation of tumor number expanded patient selection; 1-, 3-, and 5-year survival rates are reported to be $92.2,82.6$, and $79.9 \%$, respectively. ${ }^{215}$ In the selection of HCC patients for LT, the University of Tokyo has adopted the 5-5 rule, i.e., HCC $\leq 5 \mathrm{~cm}$ and $\leq 5$ in number, and a recurrencefree survival rate of 94\% after LT was achieved. ${ }^{216}$ Kyoto University further extended the number of tumors to 10 with serum PIVKA-II levels $\leq 400 \mathrm{mAU} / \mathrm{mL}$; the resultant 5-year survival rate was $86.7 \% .{ }^{217}$ At Kyushu University, a 5-year survival rate of $82.7 \%$ was achieved in patients with HCCs $\leq 5 \mathrm{~cm}$ and serum PIVKA-II levels $<300 \mathrm{mAU} / \mathrm{mL}{ }^{218}$ In a study involving 49 centers and 653 patients in Japan, patients with HCCs beyond the Milan criteria but with serum AFP levels $\leq 200 \mathrm{ng} / \mathrm{mL}$ and serum PIVKA-II levels $\leq 100 \mathrm{mAU} / \mathrm{mL}$ had a 5-year disease-free survival rate of $84.3 \% .^{219}$

LDLT has been proposed as an ideal setting for exploring expanded indications for HCC, considering a lack of graft allocation and priority policies. Moreover, the graft of a live donor is a personal gift. If the posttransplant outcomes of several eligible criteria beyond the Milan criteria for LDLTs are comparable to those with the Milan criteria, expanded indications can be accepted as long as the safety of the live donor is ensured. The 5-year overall and disease-free survival rates of expanded indications beyond the Milan criteria for LDLTs exceed 80\% and $70 \%$, respectively. ${ }^{220-222}$

The outcomes of live donors ( $n=2,872$ published cases) from 
Korea are excellent. ${ }^{223-228}$ The risks and benefits of LDLT should take into account both the donor and recipient; this concept is known as "double equipoise." The associated probabilities of death and life-threatening complications in LDLT for healthy donors are reported to be $0.3 \%$ and $<2 \%$, respectively. ${ }^{220-222,229}$ Because of the complexity of the procedure, LDLT must be restricted to centers of excellence in hepatic surgery and LT to minimize donor risk and maximize recipient outcome. Careful attention should be given to the psychosocial aspects of live donors.

\section{Salvage liver transplantation}

Salvage liver transplantation (SLT) is promoted as a potential curative treatment strategy for HCC recurrence following primary liver resection with curative intent. ${ }^{230}$ This strategy may reduce disease progression in patients waiting for LT and may also reduce the number of transplants required. An intentionto-treat analysis shows that LT for patients with small resectable HCCs yields survival outcomes superior to those of liver resection. ${ }^{231-233}$ Nevertheless, patients with small resectable HCCs are offered liver resection rather than LT because of the aforementioned organ shortage. ${ }^{234}$

Patients with HCCs beyond as well as within the Milan criteria should be initially treated with liver resection and can later be salvaged with LT if they develop recurrent HCC within the Milan criteria and is not too aggressive. ${ }^{153}$ The selection criteria for SLT are same as those for primary LT. ${ }^{153,154,232,235-239}$ This is because the risk factors for HCC recurrence after SLT are similar to those of primary LT. ${ }^{236,240}$ Significant independent risk factors for HCC recurrence after SLT include microscopic vascular invasion, poor differentiation, satellite nodules, high AFP level, and tumor size and number. Extension beyond the Milan criteria and avoiding risk factors are also important issues regarding SLT. Furthermore, prophylactic or preemptive SLT before HCC recurrence for patients with risk factors for recurrence after hepatic resection remains controversial in light of the organ shortage. Another concern is the potential for surgical difficulty following prior resection and postoperative complications, which may negate the benefit of an SLT. Meta-analyses and systemic reviews ${ }^{154,234,241}$ show that the recipient outcomes of SLT are similar to those of primary LT with respect to overall and disease-free survival.

For patients who develop recurrent HCC after primary hepatic resection but have a very low probability of receiving DDLT before tumor progression, salvage LDLT could be a curative treatment option like primary LT. The indications and selection criteria for salvage LDLT are same as those for primary LDLT. ${ }^{153,236-239}$

\section{Posttransplantation immunosuppression}

A calcineurine inhibitor-based immunosuppressant is generally administered in HCC patients after LT. ${ }^{242}$ Sirolimus was recently reported to suppress HCC recurrence via an antiprolifera- tion effect ${ }^{243,244}$ contributing to improved survival. ${ }^{245}$ However, this finding must be corroborated by additional studies.

\section{Recommendations (Table 2)}

1. DDLT is the first-line treatment for patients with singlenodular HCC $<5 \mathrm{~cm}$ in diameter or 3 or fewer $\leq 3$ nodules $\leq 3 \mathrm{~cm}$ in diameter (Milan criteria), which are not indicated for resection (A1).

2. Locoregional therapies (local ablation or TACE) are recommended if the timing of transplantation is not predictable (B1).

3. Downstaging (e.g., with TACE) can be considered for HCCs exceeding the criteria for transplantation (C2).

4. LDLT is an effective alternative to deceased donor transplantation (B1).

5. An expanded indication for transplantation beyond the Milan criteria can be considered in HCC cases without definitive vascular invasion or extrahepatic spread, if other effective treatment options are not inapplicable (C2).

6. Salvage transplantation can be indicated for recurrent HCC after resection according to the same criteria as for first-line transplantation (B1).

\section{LOCOREGIONAL THERAPIES}

Locoregional therapies are widely performed as nonsurgical treatments for HCC because of their convenience and relatively lower invasiveness. Although TACE can be considered a locoregional therapy in a broad sense, it will not be discussed here. RFA and percutaneous ethanol injection therapy (PEIT) are the current standard local therapies, while others such as microwave ablation, laser ablation, cryoablation, acetic acid injection therapy, and high-intensity focused ultrasound are currently undergoing clinical trials.

The indications for locoregional therapies include patients with a single HCC nodule $\leq 5 \mathrm{~cm}$ or up to 3 nodules $\leq 3 \mathrm{~cm}$, although they vary among studies. The effectiveness of local therapies depends on tumor size, while complete tumor necrosis rates are reported to exceed $80 \%$ for tumors $<3 \mathrm{~cm}$. ${ }^{246}$ Predictors of survival include initial complete tumor necrosis, Child-Pugh score, tumor size and number, and baseline serum AFP level. In particular, local therapies are very effective for Child-Pugh class A patients with single small HCC nodules $<2 \mathrm{~cm}$, although surgical resection is the standard treatment for such tumors. ${ }^{82}$ Some researchers assert local therapies should be the primary treatments for these tumors because of the favorable tumor response; however, this remains controversial. ${ }^{247}$ Local therapies are less effective for larger tumors, but efforts to improve them are being made. Contraindications for local therapies include corrected platelet count $<50 \times 10^{3} / \mathrm{mm}^{3}$ and low prothrombin time $(\leq 50 \%)$. 


\section{Radiofrequency ablation}

RFA is currently the most widely used ablation technique for the treatment of HCC. Very fast alternating currents (460 to 500 $\mathrm{kHz}$ ) flow in the vicinity of radiofrequency electrodes, inducing internal friction among molecules. The internal heat generated by the internal friction can evoke tissue necrosis. Heating to $60^{\circ} \mathrm{C}$ can cause almost immediate protein denaturation and destruction of cell membranes followed by coagulative necrosis. Heating to $45^{\circ} \mathrm{C}-50^{\circ} \mathrm{C}$ for $\geq 3$ minutes can also cause similar necrotic effects.

The main advantage of RFA is that fewer treatment sessions are required to achieve complete tumor necrosis. For HCC nodules $>2 \mathrm{~cm}$, RFA results in a higher complete tumor necrosis rate than PEIT; $;^{248-251}$ most procedures were performed via a percutaneous approach, although a laparoscopic or open surgical approach may be required in some instances. Initial complete tumor necrosis rates on imaging studies are reported to exceed 96\% and if RFA procedures are repeated for residual viable tumors, a complete tumor necrosis rate of almost 100\% can be achieved. ${ }^{250}$ However, the estimated 3-year local tumor progression rate after RFA ranges widely from $0.9 \%$ to $21.4 \% .{ }^{247,252,253}$

Despite these favorable outcomes, RFA has some disadvantages. First, the risk of major adverse events is usually higher than that with PEIT, particularly when the tumors are located near the liver hilum or a major abdominal organ such as the large intestine. In addition, the heat sink effect may hinder effective transmission of heat energy to the tumor in cases in which the tumors are adjacent to relatively large intrahepatic vessels. ${ }^{251,254,255}$ However, the risk of thermal injury to the adjacent abdominal organs can be overcome by inducing artificial ascites. ${ }^{256}$ Another major limitation of RFA is that HCC nodules $<2 \mathrm{~cm}$ may not be visible on conventional ultrasonography. However, recent applications of ultrasound contrast agents and fusion imaging techniques have broadened the indications for RFA to such cases. ${ }^{257,258}$

The mortality rate due to procedure-related complications after RFA is reported to be $0.1 \%-0.5 \%$, and the major complication rate after RFA is $<5 \% .{ }^{247,254,255}$ Major complications include needle tract seeding, hemoperitoneum, hemothorax, liver abscess, massive infarction of liver parenchyma, intestinal perforation, pneumoperitoneum, etc. ${ }^{253}$ The long-term survival outcomes of HCC patients can vary after RFA with respect to tumor size. For Child-Pugh class A cirrhotic patients with tumors $<2 \mathrm{~cm}$, the 3- and 5-year overall survival rates after RFA are reported to be approximately $90 \%$ and 65\%-70\%, respectively; ${ }^{247,252,253}$ meanwhile, those for tumors $2-5 \mathrm{~cm}$ are $65 \%-75 \%$ and 50\%, respectively. ${ }^{252,253}$ The 10 -year overall survival rate of Child-Pugh class A patients with single HCC $<3 \mathrm{~cm}$ is $41.3 \%$. ${ }^{253}$

In four RCTs comparing RFA and PEIT for patients with HCC, ${ }^{249-251,259,260}$ patients treated with RFA showed better local tumor response and overall survival outcomes than those treated with PEIT. A meta-analysis of these four RCTs also demonstrates that the 3-year overall survival rate of RFA is significantly higher than that of PEIT. ${ }^{261}$ Nevertheless, for patients with HCC $<2 \mathrm{~cm}$, there is no significant difference in survival outcomes between RFA and PEIT. Therefore, further prospective controlled studies are required.

As it is difficult to compare observational studies evaluating the long-term survival outcomes of RFA and surgical resection, it is not adequate to draw a definite conclusion regarding the superiority of either treatment. Three RCTs have recently been published on this topic. There were no significant differences in the survival outcomes between the two treatments in one RCT for solitary HCC $<5 \mathrm{~cm}$ and another RCT for single or double HCCs $<4 \mathrm{~cm} .{ }^{262,263}$ However, the other RCT for HCC diagnosed according to the Milan criteria indicates the superiority of surgical resection over RFA with respect to 3- and 5-year overall survival rates $(\mathrm{p}<0.001) .{ }^{264}$ Nevertheless, such results should be interpreted cautiously, as RFA is most effective for tumors $<3$ $\mathrm{cm}$. A meta-analysis of these three RCTs shows that the complication rate was usually higher in the resection group than the RFA group ( $\mathrm{p}=0.002)$ and that hospitalization duration was significantly longer in the resection group than the RFA group $(p<0.001) .{ }^{265}$ Nevertheless, it is not possible to draw a definite conclusion about the overall or disease-free survival rate of HCC patients on the basis of a meta-analysis of the three RCTs mentioned above, because the indications differed substantially among studies. ${ }^{265}$ In contrast, RFA may be unfeasible because of the tumor location in some cases, making surgical resection more useful. ${ }^{263}$ As a strategy combining the advantages of these treatment modalities for small HCCs, i.e., the lower invasiveness of RFA and better curative potential of hepatic resection, primary RFA and subsequent resection in cases of failed RFA can be considered. A recent decision model analysis shows there was no difference in the survival outcomes of patients with single HCC smaller than $2 \mathrm{~cm}$ between the combined strategy and resection monotherapy. ${ }^{266}$

For intermediate-sized HCCs (i.e., 3-5 cm), the local recurrence rates after RFA are reported to range from 30\% to 50\%, ${ }^{252}$ and combined treatment of TACE and RFA can be considered for these tumors. The difference in the 3-year overall survival rates between combined treatment and RFA monotherapy is $<10 \%$ for tumors smaller than $3 \mathrm{~cm}^{267,268}$ but is much greater for HCCs 3-5 cm in diameter. ${ }^{268}$ Meta-analyses of RCTs also show similar results. ${ }^{269,270}$

No RCT has compared re-resection and RFA for recurrent HCC following hepatic resection. However, a recent retrospective analysis shows that the 2- and 5-year overall survival rates after re-resection and RFA are 90\% vs 96\% and 72\% vs 83\%, respectively; the differences are not statistically significant. ${ }^{271}$ Another retrospective study shows similar results. ${ }^{272}$ In those studies, baseline liver functional status might have been unfavorable in the RFA group compared to the surgical group. Consequently, 
RFA appears comparable to re-resection for the treatment of recurrent HCC following hepatic resection, although further investigation is necessary to confirm this. ${ }^{273}$

\section{Percutaneous ethanol injection}

PEIT is widely used in the treatment of HCC because it is relatively convenient to perform and adverse reactions are infrequent. However, PEIT has been largely supplanted by RFA recently, mainly because PEIT should be performed repetitively in contrast to RFA and it is difficult to obtain complete necrosis for tumors larger than $3 \mathrm{~cm}$. PEIT is now usually reserved for patients with $\leq 3$ nodules $\leq 3 \mathrm{~cm}$ in diameter.

As a special consideration, PEIT can be performed to treat perivascular tumors to reduce the heat sink effect of RFA. However, the risk of biliary stricture is not eliminated by PEIT if the tumors are located in the liver hilum. ${ }^{274}$ The reported rates of tumor necrosis after PEIT range between 66\% and $100 \% .^{249-251,259}$ In particular, the therapeutic efficacy of PEIT largely depends on the tumor size; the rate of tumor necrosis decreases with increasing tumor size. A rate of tumor necrosis $\geq 90 \%$ is reported for tumors smaller than $2 \mathrm{~cm}$, but the rate decreases to approximately $50 \%$ for $3-5-\mathrm{cm}$ tumors. Local tumor progression rates after PEIT range between 24\% and 34\%, although there is no consensus on the definition of local tumor progression. ${ }^{275-277}$ For Child-Pugh class A patients with solitary tumors smaller than $2 \mathrm{~cm}$, the 3- and 5-year overall survival rates are 70\%-80\% and $\geq 50 \%$, respectively. For tumors $2-3 \mathrm{~cm}$ in diameter, the 3 -year overall survival rate ranges from $47 \%$ to $64 \%$. $^{249,259}$

For cirrhotic patients with Child-Pugh class A or B and a solitary tumor smaller than $3 \mathrm{~cm}$, several comparative studies show no definite differences in the survival outcomes between PEIT and surgical resection. ${ }^{278,279}$ In particular, an RCT comparing PEIT and surgical resection targeting 76 patients who had one or two HCC nodules $\leq 3 \mathrm{~cm}$ reports no difference in the survival rates or local recurrence rates between treatment groups. ${ }^{279}$ However, it is very difficult to reach a definite conclusion based on a single RCT. Furthermore, the sample size of that RCT was calculated on the basis of the tumor recurrence rate and not the survival rate. The 5-year survival rates differed greatly-46\% and $81.8 \%$ in the PEIT and resection groups, respectivelyeven though there was no significant difference in the overall survival rates of the two treatments. Therefore, additional welldesigned prospective controlled studies are necessary to reach a definite conclusion.

\section{Other locoregional therapies}

Other local therapies are currently under investigation, including microwave ablation, cryoablation, high-intensity focused ultrasound, laser ablation, and holmium injection therapy among others. An important advantage of microwave ablation over RFA is that treatment efficacy is less affected by vessels lo- cated near the tumor. In addition, effective ablation can be expected even for tissues with low electrical conductivity, and the simultaneous application of multiple electrodes is technically feasible with this technique. ${ }^{280,281}$ Despite its potential advantages, no RCT has compared microwave ablation and RFA with respect to clinical usefulness.

Furthermore, no RCTs have evaluated the other ablation techniques mentioned above; even single-arm studies do not demonstrate any additional benefits over RFA with respect to response or survival outcomes. Therefore, clinical indications for these various ablation techniques are currently uncertain.

\section{Recommendations (Table 2)}

1. RFA provides survival comparable to that of resection in patients with single-nodular HCCs $\leq 3 \mathrm{~cm}$ in diameter (A2).

2. RFA is superior to PEIT in terms of anticancer effect and survival (A1). For HCCs $\leq 2 \mathrm{~cm}$ in diameter, PEIT can be considered if RFA is unfeasible, because the outcomes of both modalities are similar (A2).

3. Survival outcomes can be improved by combining TACE and RFA compared to RFA alone in patients with tumors 3-5 $\mathrm{cm}$ in diameter if resection is unfeasible (A2).

\section{TACE AND OTHER TRANSARTERIAL THERAPIES}

Most HCC patients are unresectable at the time of diagnosis because of portal hypertension, poor liver functional status, multiplicity of tumors, portal vein tumor invasion, inability to secure sufficient resection margin around the tumors, old age, and severe comorbidities. ${ }^{282}$ TACE is the most commonly used nonsurgical treatment modality for these patients; meanwhile, tumor necrosis can be achieved by the combined effects of antitumor chemotherapy and selective ischemia of tumor tissue. ${ }^{13,282,283}$ TACE is the most widely practiced primary treatment modality for HCC in Asia and North America. ${ }^{284}$ TACE can be classified as conventional TACE using lipiodol and drug-eluting bead TACE (debTACE). ${ }^{285,286}$ It is important to note that TACE should be distinguished from transarterial embolization, which uses only embolic material, and HAIC, which uses only antitumor chemotherapeutic agents. ${ }^{287,288}$

\section{Conventional TACE}

The TACE procedure involves mixing chemotherapeutic agents such as doxorubin, cisplatin, and mitomycin with iodized oil and injecting the mixture into the feeding artery as an emulsion. This is followed by arterial embolization using gelatin sponge particles, polyvinyl alcohol particles, or microspheres, which induce selective tumor ischemia. The most important technique for maximizing the antitumor effect and minimizing liver toxicity when performing TACE is to superselect the feeding arteries of tumors as distal as possible. ${ }^{289}$ Regarding the repetition strategy of TACE, on-demand repetitions to treat 
the residual or recurrent tumors can minimize the incidence of procedure-related liver toxicity, which is therefore preferable to on-schedule regular repetitions every 1-2 months.

Several RCTs and meta-analyses confirm compared to supportive treatments, TACE results in more favorable tumor response, time to progression, and survival outcomes in patients with unresectable HCC. ${ }^{77,290,291}$ A recent prospective cohort study by the Japanese Liver Cancer Study Group reports that the 1-, 3-, 5-, and 7-year survival rates of 8,510 patients who underwent TACE were 82\%, 47\%, 26\%, and 16\%, respectively; for tumors larger than $5 \mathrm{~cm}$, the 1-, 3-, and 5-year survival rates were $63 \%, 30 \%$, and $16 \%$, respectively. ${ }^{292}$ In a recent prospective multicenter study performed in 27 Japanese and South Korean centers, the complete or partial remission rate according to the modified Response Evaluation Criteria in Solid Tumors (mRECIST) criteria was 73\% and the 2-year overall survival rate was $75 \%$; these figures are higher than those previously reported in the literature. ${ }^{293}$ In that study, grade 3-4 severe toxicities after TACE included elevated serum alanine aminotransferase level in $36 \%$ of patients, thrombocytopenia in $12 \%$, and abdominal pain in $4 \%$, while mild fever occurred in 57\%. In another retrospective analysis, postembolization fever $>38.0^{\circ} \mathrm{C}$ occurred after TACE in 20\% of patients; it occurred more frequently in patients with tumors $>5 \mathrm{~cm}$ and was a poor prognostic factor for survival. ${ }^{294}$ Even though TACE can be performed safely in general, severe complications such as irreversible hepatic failure occur in 3\% of patients and postembolization syndrome, which is characterized by transient fever and abdominal pain, occurs more frequently, affecting $60 \%-80 \%$ of patients. ${ }^{295}$ The best tumor response and the lowest complication rates after TACE can be expected for patients with favorable performance status, with nodular HCCs, and without traces of vascular invasion. Future RCTs should evaluate the survival benefits of TACE for patients with unfavorable prognostic factors such as poor performance status, major portal vein tumor invasion, Child-Pugh class C, and extrahepatic metastasis.

Local tumor response after TACE can vary substantially according to the size and number of tumors as well as patterns of tumor growth, such as tumor encapsulation and vascular invasion. The complete remission rate is quite low for large or multiple tumors despite multiple TACE sessions. However, in cases with small tumors, complete tumor necrosis can be obtained in more than 50\% of cases after superselective TACE. ${ }^{296}$ For patients with Child-Pugh class A and surgically resectable HCC <4 $\mathrm{cm}$, the 5-year overall survival rate after subsegmental TACE is reported to exceed 50\%. ${ }^{289,292}$ A prospective cohort study from Korea comparing surgical resection after primary TACE with TACE monotherapy published a decade ago reports that the survival rates were similar between the two treatment groups in cases in stage T3. In addition, the survival rate of the TACE group in stage T1 and T2 cases was similar to that of the surgical resection group if iodized oil was compactly retained within the tumor. ${ }^{297}$ In a recent prospective cohort study on BCLC stage A patients for whom resection or ablation could not be performed, the 1-month complete remission rate according to the mRECIST criteria were $67 \%$ and the 3-year overall survival rate was $80 \% .{ }^{298}$ In another recent retrospective study comparing resection, RFA, and TACE as initial treatments for single small HCC $<3 \mathrm{~cm}$ in diameter, the unadjusted 5-year overall survival rate of the TACE group was the lowest at 74.2\%. However, after adjusting for liver functional status, thrombocytopenia, varix, etc., the differences in the survival outcomes among the groups lost statistical significance. ${ }^{299}$ Taking the potential selection bias of the abovementioned studies into account, TACE can be considered an alternative treatment with curative intent if a patient rejects surgical treatment, is high risk for surgery, or is contraindicated for RFA.

Portal vein tumor invasion occurs in approximately 30\% of HCC patients in Korea. ${ }^{13}$ According to the AASLD practice guidelines, systemic chemotherapy with sorafenib is the standard primary treatment for HCC invading the portal vein. ${ }^{57}$ However, in practice, this recommendation is not routinely followed by physicians, because the expected survival benefits are modest. ${ }^{284}$ Therefore, further investigations of effective alternative treatments are required. When TACE is performed for HCC patients with good hepatic functions but portal vein tumor invasion, the risk of hepatic functional deterioration after TACE is reported to be acceptably low..$^{300-302}$ The 1- and 3-year overall survival rates of such patients after repeated TACE range from 25\%-35\% and 9\%-10\%, respectively. ${ }^{292,303,304}$ In a prospective study targeting HCCs invading the major portal vein, the median survival period of the TACE-treated group (5.0 to 5.1 months) was 2 to 2.5 months longer than those of the supportively treated group; however, the difference was not statistically significant probably because of the small number of patients included. ${ }^{291,302}$ However, a recent study from Korea reports that the median survival rate of TACE-treated HCC invading the major portal vein is 22 to 30 months for a subgroup of patients with nodular tumor growth or limited tumor extent. ${ }^{302,303} \mathrm{~A}$ recent prospective nonrandomized study on unresectable HCC patients with portal vein invasion shows more favorable survival outcomes for the TACEtreated group than the supportive treatment group. ${ }^{304}$ However, the lack of randomization in that study limits the validity of this finding. Therefore, additional well-designed RCTs are required to confirm the survival benefits of TACE over supportive treatment in HCC patients with portal vein invasion. Future investigations are also required to compare the clinical effectiveness of TACE monotherapy with other monotherapies (i.e., radiation therapy, systemic sorafenib, hepatic arterial infusion chemotherapy, etc.) and combined treatments of those therapies and TACE while considering liver functional status, tumor extent, and the extent of portal vein invasion. 


\section{Drug-eluting bead TACE}

Drug-eluting beads, which are microspheres loaded with highdose doxorubicin, represent a newly developed embolic agent for tumor feeders. The major theoretical advantage of debTACE is the higher intratumor drug concentration and lower serum drug concentration due to the slow release of doxorubicin from the microspheres after embolization of the tumor feeders. In prospective clinical trials, liver toxicities and systemic adverse effects occur less frequently after debTACE than conventional TACE. ${ }^{305,306}$ In contrast, a phase II RCT on 212 HCC patients demonstrates no additional benefit of debTACE over conventional TACE with respect to 6-month complete or partial remission rate. ${ }^{305}$ However, subgroup analyses show that debTACE was superior to conventional TACE in patients with Child-Pugh class B, ECOG performance status 1, bilobar disease, or recurrent HCCs. ${ }^{305}$ In another RCT, there was no significant difference between the two treatments with respect to tumor response, time to progression, or survival period. ${ }^{307}$ The 5 -year overall survival rates of HCC patients after debTACE and conventional TACE were 38.3\% and 22.5\%, respectively. ${ }^{308,309}$

When debTACE is performed by experienced interventional radiologists, postembolization syndrome or systemic adverse effects occur less frequently. ${ }^{306,310}$ However, there is a lack of evidence confirming the long-term survival benefits of debTACE compared to conventional TACE, thus warranting further phase III RCTs.

\section{Transarterial radioembolization}

TARE involves the injection of implantable radioactive microspheres into tumor-feeding arteries in order to expose the tumor to highly concentrated radiation while protecting the normal parenchyma. Yttrium-90 $\left({ }^{90} \mathrm{Y}\right)$ is the most commonly used radioisotope and emits high-energy and pure $\beta$-rays with a halflife of 64.2 hours, and mean and maximum tissue penetration of 2.5 and $11 \mathrm{~mm}$, respectively. The microspheres available for ${ }^{90} \mathrm{Y}$ infusion are about $35 \mu \mathrm{m}$ in diameter and are made of resin or glass. The small size of the injected microspheres and their concentration at the hypervascular HCC minimize the embolic effect on surrounding tissue. Preprocedural angiography and ${ }^{99} \mathrm{mTc}$-labeled macroaggregated albumin scan are required to decide on the treatment site, determine the radiation dose, and detect and calculate the degree of shunting to the lungs and any other extrahepatic organs.

In a prospective single-arm phase II study of 52 patients with intermediate or advanced HCC treated with TARE, the objective tumor response rate (i.e., complete or partial remission rate) was $40.4 \%$, and the median survival period was 15 months. ${ }^{311}$ In another large retrospective cohort study, the median survival periods of patients with and without portal vein invasion were 10 and 15.3 months, respectively; the difference was statistically significant. The median survival periods of patients with Child-
Pugh class A and B were 17.2 and 7.7 months, respectively; the difference was also statistically significant. ${ }^{312,313}$ In a recent prospective multicenter study performed in Korea on 40 HCC patients in BCLC stage B or C, the 3-month tumor response rate was 57.5\% and the 3-year overall survival rate was 75\%. ${ }^{314}$

The most frequent adverse effect after ${ }^{90} \mathrm{Y}$ TARE is transient fatigue. However, postembolization syndrome is less likely to occur than in conventional TACE, because the embolic effect of ${ }^{90} \mathrm{Y}$ TARE is not as strong. Therefore, ${ }^{90} \mathrm{Y}$ TARE can be performed safely for patients with portal vein tumor thrombosis. Elevated serum bilirubin levels occur in $20 \%$ of patients, and the mortality rate within 1 month ranges from $0 \%$ to $3 \% .^{31-313}$ Severe complications such as radiation pneumonitis and gastroduodenal ulcer can occur in the event of inadvertent embolization into the extrahepatic organs. Therefore, ${ }^{90} \mathrm{Y}$ TARE requires meticulous precautions and experience performing radioembolization.

In summary, no RCT has compared ${ }^{90} \mathrm{Y}$ TARE with other standard treatments with respect to long-term survival outcomes and complication rates. Therefore, it is difficult to suggest indications for ${ }^{90} \mathrm{Y}$ TARE in HCC patients. However, the incidence of postembolization syndrome and systemic adverse effects with ${ }^{90} \mathrm{Y}$ TARE are relative low, and the tumor response rates are similar to those of conventional TACE. Accordingly, a phase III RCT is required to determine the indications, cost-effectiveness, and survival outcomes for this new type of treatment.

\section{Recommendations (Table 2)}

1. TACE is recommended for patients with good performance status without major vascular invasion or extrahepatic spread who are ineligible for surgical resection, liver transplantation, RFA, or PEIT (A1).

2. TACE should be performed through tumor-feeding vessels using selective/superselective techniques to maximize antitumor activity and minimize hepatic damage (B1).

3. Chemoembolization using drug-eluting beads results in less systemic adverse events and has similar therapeutic efficacy compared with conventional TACE (B2).

4. In case of portal vein invasion, TACE can be considered for patients with localized tumors and well-preserved liver function (B2).

\section{EXTERNAL-BEAM RADIATION THERAPY}

External-beam radiation therapy (EBRT) for the treatment of HCC is commonly used for lesions that are surgically unresectable and not amendable with other local modalities. Child-Pugh class A or upper B are criteria for EBRT. The reported overall response rates and median survival after EBRT are 40\%-90\% and 10-25 months, respectively. ${ }^{315}$ EBRT requires computerized radiation therapy planning by $\mathrm{CT}$, and the liver volume receiving $\geq 30$ Gy must be limited to $\leq 60 \%$ of the total liver volume in 
three-dimensional radiotherapy planning-based dose-volume analysis. ${ }^{316}$ For hypofractionated EBRT consisting of $\leq 10$ fractions, the normal liver volume receiving $<15$ Gy must be $\geq 700$ $\mathrm{mL}^{317}$ and the dose to the normal liver volume excluding the tumor must be limited to $\leq 28$ Gy (corrected to 2 Gy per fractionequivalent dose). ${ }^{318}$

EBRT can be used for patients with HCC who are unsuitable for surgical resection, LT, RFA, PEIT, or TACE. In most centers, hypofractionated radiotherapy including stereotactic ablative radiation therapy is used to treat patients with Child-Pugh $\mathrm{A}$ or superb B liver function and adequate normal liver volume; the 2-year local control and survival rates are reported to range from $70 \%$ to $100 \%$ and $50 \%$ to $75 \%$, respectively. ${ }^{319-325}$ In particular, stereotactic ablative radiation therapy for the treatment of $\leq 3$ lesions and lesions with a cumulative diameter $\leq 6$ $\mathrm{cm}$ results in local control rates exceeding 90\%. ${ }^{319,323-325}$ Proton therapy for HCC results in a 2-year local control rate from 75\% to $96 \%$ and a 5 -year survival rate from $23 \%$ to $44 \%{ }^{199,326-329}$

An advantage of EBRT is that it can be performed safely regardless of the presence of portal vein invasion by the tumor. ${ }^{330-344}$ One study reports that when EBRT was used for patients unsuitable for TACE owing to severe tumor-induced arteriovenous shunts, $20 \%$ of these patients were able to undergo TACE successfully after radiation therapy-induced vascular occlusion. ${ }^{345}$ A meta-analysis reports that the use of TACE in combination with EBRT improves the 3-year survival rate by 10\%-28\% compared to TACE monotherapy. ${ }^{346}$ Meanwhile, the results of controlled studies comparing combination treatments are anticipated. Moreover, the addition of EBRT for HCC after incomplete TACE is reported to result in a complete response rate of 20.9\%. ${ }^{347}$ In a Korean multicenter retrospective cohort analysis, 78.4\% of patients received TACE before receiving EBRT. $^{348}$ Another recent study shows that TACE combined with EBRT for resectable HCC with portal vein invasion resulted in a superior median survival of 12.3 months versus 10.0 months for the surgical group. ${ }^{349}$ Furthermore, combined treatment for HCC patients with inferior vena cava invasion also resulted in a superior median survival period of 11.7 months in comparison with the historical cohort who received TACE alone. ${ }^{350}$ The sequential combination of EBRT 2 weeks after TACE may be complicated by liver dysfunction; however, Common Terminology Criteria of Adverse Events grade $\geq 3$ liver dysfunction is reported in only $2.5 \%$ of all patients. ${ }^{351}$

In one study, regional chemotherapy with 5-fluorouracil and cisplatin combined with EBRT for locally advanced HCC resulted in a 3-year survival rate of $24.1 \%$ and a median survival period of 13.1 months. ${ }^{352}$ The same institution reports a median progression-free survival of 4.5 months and overall survival of 9.8 months after TACE followed by EBRT and concurrent intraarterial 5-fluorouracil for locally advanced HCC with portal vein invasion and intrahepatic metastases. ${ }^{353}$ In another study, the addition of EBRT to intra-arterial 5-fluorouracil and sub- cutaneous interferon treatment for 40 patients with advanced HCC and portal vein invasion significantly improved the time to progression from 4.0 to 6.9 months and the median survival from 9.1 to 12.0 months. ${ }^{354}$ EBRT can also be considered a local neoadjuvant treatment for large HCCs with the aim of improving resectability. Accordingly, it has been reported that surgery can be performed safely post-EBRT, resulting in an effective response to neoadjuvant radiotherapy. ${ }^{355}$ In addition, EBRT can be considered a bridging treatment for patients awaiting liver transplantation. ${ }^{199,200,319}$ Nevertheless, a controlled prospective comparative study is required to establish the role of EBRT as a new adjuvant treatment. EBRT can also be used as a secondline treatment for recurrent HCC after various nonsurgical treatments including TACE. ${ }^{38,356,357}$

EBRT is also effective for relieving symptoms such as cancer pain. ${ }^{315,358}$ In patients with jaundice presenting with biliary obstructions due to the progression of HCC, EBRT has been demonstrated to successfully reduce tumor size and relieve symptoms; accordingly, EBRT is also expected to improve the survival in these patients. ${ }^{359,360}$ In HCC patients with abdominal lymph node metastases, EBRT results in response rates of approximately $80 \%{ }^{361-364}$ with improved median survival time. ${ }^{365}$ In HCC patients with symptomatic bone metastases, EBRT is reported to relieve pain in 75\%-99\% of patients ${ }^{366-370}$ as well as symptoms in patients with brain metastases from HCC. ${ }^{371}$ Moreover, in a previous study, EBRT doses from 30 to 50.7 Gy for spinal cord compression from vertebral metastases resulted in ambulatory rates of $85 \%$ and $63 \%$ at 3 and 6 months, respectively. ${ }^{372}$ In two other studies, EBRT for lung metastases resulted in response rates from 60\% to $70 \%$ while symptom relief was observed in $90 \%$ of symptomatic patients. ${ }^{361,373}$

\section{Recommendations (Table 2)}

1. EBRT can be performed in HCC patients if liver functions are Child-Pugh class A or superb B and the irradiated total liver volume receiving $\geq 30$ Gy is $\leq 60 \%$ (B1).

2. EBRT can be considered for HCC patients ineligible for surgical resection, liver transplantation, RFA, PEIT, or TACE (C1).

3. EBRT can be considered for HCC patients who show incomplete response to TACE when the dose-volume criteria in Recommendation 1 are met (B2).

4. EBRT can be considered for HCC patients with portal vein invasion when the dose-volume criteria in Recommendation 1 are met (C1).

5. EBRT is performed to alleviate symptoms caused by primary HCC or its metastases (B1).

\section{SYSTEMIC THERAPIES}

\section{Sorafenib}

Sorafenib is a multi-tyrosine kinase inhibitor targeting vascular endothelial growth factor receptor 2, platelet-derived 
growth factor receptor, Raf-1, and c-kit. Sorafenib was the first approved molecular targeted agent for the treatment of HCC. In the SHARP study, a global phase III trial, the median survival of HCC patients with portal vein tumor invasion or extrahepatic metastasis treated with sorafenib was 10.7 months, which was significantly longer than 7.9 months in patients who received placebo (HR, $0.69 ; 95 \%$ CI, 0.55 to $0.87 ; p=0.00058$ ). ${ }^{374}$ The time to progression in the sorafenib group was 5.5 months, which was also significantly longer than 2.8 months in the control group. ${ }^{374}$ In an Asia-Pacific phase III trial that included Korean patients with unresectable HCC, patients who received sorafenib had a significantly longer median survival ( 6.5 months) than that of the control group (4.2 months; HR, 0.68; 95\% CI, 0.50 to $0.93 ; p=0.014) .{ }^{375}$ The median survival of patients treated with sorafenib was consistently reported to be approximately 10 months in the following 3 randomized controlled phase III trials testing novel molecular targeted agent in which sorafenib treatment was the control group. ${ }^{376-378}$ On the basis of the results of clinical trials, sorafenib is currently the only molecular targeted agent proven to prolong survival in advanced HCC patients.

The abovementioned two phase III trials for sorafenib (i.e., the SHARP and Asia-Pacific trials) recruited HCC patients with Child-Pugh class A and ECOG performance status 0-2. In clinical practice, the safety and efficacy of sorafenib are reported to be comparable between Child-Pugh class A and B patients; ${ }^{379-382}$ however, the prognosis is reported to differ with respect to the presence of ascites and Child-Pugh score. ${ }^{383}$ Thus, although sorafenib can be used cautiously in patients with decreased liver function, more controlled studies are necessary. The most common adverse event related to sorafenib treatment is hand-foot syndrome reaction (HFSR), followed by fatigue, skin rash, anorexia, weight loss, hypertension, and alopecia. As HFSR tends to be decreasing sptontaneously after 3 months of treatment, it is important to continue therapy through patient education and proper management; for example, creams containing urea may be helpful for preventing dryness of the hands and feet. Furthermore, it is recommended to remove thick calluses, wear comfortable shoes with cushioning, avoid hot water, and take analgesics if necessary. ${ }^{384}$ Sorafenib is primarily used in advanced HCC patients with vascular invasion (i.e., the portal vein, hepatic vein, or inferior vena cava) or extrahepatic metastasis. It can also be used in HCC patients in whom locoregional therapies have failed or are not indicated. Although there is no clear definition of TACE failure or refractoriness, if HCC progresses and the tumor stage advances despite repeated applications of TACE (i.e., 3 times within 6 months) for residual or recurrent tumors after the initial TACE, sorafenib might be indicated on the basis of the concept of TACE failure or refractoriness. ${ }^{355-387}$ Regarding combination therapy with TACE and sorafenib in intermediate-stage HCC, a single-center phase II study shows longer survival and time to progression in combination therapy than a historic control group treated with TACE monotherapy; ${ }^{388}$ while another phase II study, the SPACE trial, does not show the beneficial outcomes of combination therapy; ${ }^{389}$ the former has the limitation of being a single-arm study, while the latter has drawbacks of study design, specifically not "on-demand" TACE but "scheduled" TACE and an endpoint of overall survival in patients with intermediate-stage HCC, who are expected to have favorable long-term prognosis. On the basis of the proven safety of combination therapies in both of these trials, there is an ongoing phase III trial of combination therapy with TACE and sorafenib in advanced HCC.

\section{Recommendations (Table 2)}

1. Sorafenib is indicated for HCC patients with very wellpreserved liver function (i.e., Child-Pugh class A), good performance status, and regional lymph node or extrahepatic spread or patients with tumor progression on other therapies (A1).

2. Sorafenib is recommended for HCC patients with very wellpreserved liver function (i.e., Child-Pugh class A), and good performance status, and vascular invasion (A2).

3. Sorafenib is considered for HCC patients with preserved liver function (i.e., Child-Pugh class superb B) and good performance status if the above conditions (1 and 2) are satisfied (B1).

\section{Cytotoxic chemotherapy}

Sorafenib is recommended as a first-line systemic agent; however, despite several clinical trials, no second-line agent is proven to be effective in cases in which sorafenib fails or intolerance. Cytotoxic chemotherapy can be considered in cases of HCC progression on sorafenib or drug intolerance. ${ }^{390-392}$ Most cytotoxic chemotherapeutics have shown poor response and no survival benefit through RCTs. Even though some agents result in a relatively good antitumor response, it is unrealistic to perform a multicenter prospective RCT evaluating efficacy and safety because of lack of study funding/sponsership.

Despite the widespread use of doxorubicin, the response rate is less than 20\%. ${ }^{393-395}$ Satisfactory results have not been obtained with single agents such as 5-fluorouracil, ${ }^{396}$ gemcitabine, ${ }^{397,398}$ oxaliplatin, ${ }^{399}$ capecitabine, ${ }^{400}$ and irinotecan. ${ }^{401}$ In addition, octreotide, ${ }^{402,403}$ interferon, ${ }^{404}$ and tamoxifen ${ }^{405}$ have not shown any survival benefit for HCC.

Several combination regimens have been tried because of the modest efficacy of monotherapy. The PIAF regimen (cisplatin/ interferon $\alpha$-2b/doxorubicin/fluorouracil) shows better objective response rates (20.9\% vs $10.5 \%, \mathrm{p}=0.058)$ and median overall survival (8.67 months vs 6.83 months; RR, 0.97; $p=0.830$ ), although the difference failed to reach statistical significance. Meanwhile, hematologic toxicities were significantly more frequent in PIAF group. ${ }^{406}$ Another combination regimen, FOLFOX (oxaliplatin/fluorouracil/leucovorin) was compared with doxorubicin monotherapy. Patients receiving FOLFOX had significantly better progression-free survival (2.93 months vs 1.77 months, $\mathrm{p}<0.01)$ and disease control rate $(52.17 \%$ vs 
$31.55 \%, \mathrm{p}<0.001)$ but a nonsignificant trend toward better survival (6.4 months vs 4.97 months, $\mathrm{p}=0.07$ ). ${ }^{407}$ Some results of cisplatin-containing regimens (e.g., in combination with doxorubicin, ${ }^{408}$ capecitabine, ${ }^{409,410}$ or 5 -fluorouracil ${ }^{411}$ ) or oxaliplatincontaining regimens (e.g., in combination with gemcitabine ${ }^{412}$ or capecitabine ${ }^{413}$ ) for HCC have been reported, but the benefits of these regimens compared to monotherapy are still lacking.

Most patients with HCC are accompanied by chronic liver disease or liver cirrhosis, which can alter the metabolism of chemotherapeutics and increase their toxicity. ${ }^{414}$ Therefore, systemic chemotherapy should be reserved for patients with good performance status and liver function. In addition, great care is required to maintain the quality of life of patients receiving chemotherapeutics.

\section{Hepatic arterial infusion chemotherapy}

HAIC can directly deliver high concentrations of chemotherapeutic agents via the hepatic artery with low systemic toxicities. HAIC is usually performed for HCC with portal vein invasion and can be considered for TACE-refractory cases. Recent reports demonstrate that HAIC can also be tried for sorafenib-refractory or intolerant HCCs, although further studies are warranted. ${ }^{415,416}$ The most widely used agent in HAIC is 5-fluorouracil, which is either administered alone or with cisplatin, showing response rates from 3.8\% to 38.5\% and median survival from 5 to 19.5 months. ${ }^{417-420}$ HAIC can be administered in combination with systemic interferon. ${ }^{421,422}$ The implantable device required for HAIC can sometimes cause complications such as infection and occlusion. Nevertheless, further studies are warranted, because there is insufficient evidence demonstrating that HAIC improves survival compared to systemic chemotherapy or best supportive care.

\section{Recommendations (Table 2)}

1. Cytotoxic chemotherapy can be considered for HCC patients with advanced tumors who have well-preserved liver function and good performance status in whom sorafenib therapy has failed (C1).

\section{ADJUVANT THERAPY}

Adjuvant therapy usually refers to an additional treatment after definitive or curative therapy to prevent recurrence. As there recurrence rate 5 years after curative resection for HCC is very high at 70\% (see "Liver Resection" section), ${ }^{142,423,424}$ an effective adjuvant therapy is urgently required. There is currently no proven modality; ${ }^{58}$ neither sorafenib nor cytotoxic chemotherapy is recommended as an adjuvant therapy. Furthermore, there is no evidence that TACE prevents HCC recurrence after curative resection. ${ }^{425}$

Although one study reports that ${ }^{131} \mathrm{I}$ infusion via the hepatic artery after curative resection reduces the recurrence of HCC, no validation study was performed. ${ }^{426,427}$ Thus, this radionuclide therapy is not recommended at present. A study of activated immune cells derived from patients shows a 15\% decrease in the 3 -year recurrence rate compared to that of the control group. ${ }^{428}$ However, there has been no reliable study to corroborate this.

TACE can result in the downstaging of HCC, enabling curative resection. Even for resectable HCC, TACE can be applied prior to resection as a neoadjuvant therapy. However, there is no evidence that TACE followed by resection increases diseasefree survival compared with resection only in resectable HCC. ${ }^{429}$

\section{Recommendations (Table 2)}

1. Adjuvant TACE, sorafenib, and cytotoxic chemotherapy are not recommended for HCC patients treated with curative resection (B1).

\section{PREEMPTIVE ANTIVIRAL TREATMENT}

\section{HBV carriers}

The rate of HBV reactivation in HCC patients after cytotoxic chemotherapy varies widely from $30 \%$ to $60 \%,{ }^{430,431}$ and the subsequent mortality rate is reported to be approximately $30 \%$ of all deaths resulting from $\mathrm{HBV}$ reactivation. HBV reactivation with concomitant elevation of serum HBV DNA level or abnormality of biochemical liver function is observed in 20\%-50\% of total HBV carriers who receive immunosuppressants or cytotoxic chemotherapy for the treatment of malignancies other than HCC (e.g., breast cancer, hematologic malignancies, and other solid cancers). ${ }^{40,432-435}$ Therefore, the test for hepatitis B surface antigen (HBsAg) must be performed in patients at high risk for $\mathrm{HBV}$ infection prior to immunosuppressive therapy or cytotoxic chemotherapy. ${ }^{436}$ Antiviral drugs should be preemptively administered in HBV carriers at the onset of the cytotoxic chemotherapy or immunosuppressant administration and must be continued for at least 6 months. Although further research is required to clarify the adequate serum HBV DNA level, recurrence is more likely after the discontinuation of antiviral drugs in patients with high HBV DNA levels prior to cytotoxic chemotherapy. Therefore, in patients with HBV DNA levels $>2,000$ $\mathrm{IU} / \mathrm{mL}$ prior to cytotoxic chemotherapy, continuation of antiviral treatment should be considered until the treatment goal of chronic hepatitis B is reached. ${ }^{436}$ Most studies on preemptive antiviral treatment are limited to lamivudine, but other recently developed antiviral drugs can be used. In cases of lamivudine resistance, antiviral drugs should be replaced according to the treatment guidelines for resistance. ${ }^{47,438}$ In cases in which antiviral therapy is expected to continue for more than 12 months in particular, the antiviral drug with the minimum resistance profile should be selected. ${ }^{439,440}$ Interferon is not recommended as a preemptive treatment because of the risk of bone marrow suppression and transient aggravation of hepatitis. In the HBsAg-negative, anti-HBc-positive, and anti-HBs-positive 
patients, HBV reactivation can develop very rarely, and there is little evidence to recommend uniform preemptive treatment owing to a lack of research. ${ }^{436}$

Comparatively many studies have evaluated HBV reactivation during TACE for the treatment of HCC; HBV reactivation is reported to occur in $4 \%-40 \%$ of patients. ${ }^{430,441-445}$ According to a study comparing preemptive lamivudine treatment to a nonadministered control group during TACE, ${ }^{444}$ significant differences were observed with respect to HBV reactivation $(2.8 \%$ and $40.5 \%$ ) as well as the consequent occurrence of hepatitis (2.8\% and 19.7\%) and liver failure (0\% and 8.1\%). Hence, preemptive antiviral treatment can be considered for HBV-positive HCC patients undergoing TACE. However, differences in chemotherapeutic agents, and treatment interval and frequency may have resulted in discordant $\mathrm{HBV}$ reactivation rates. ${ }^{44-446}$ Therefore, additional research is required to determine the serum HBV DNA levels and biochemical liver function test levels that require preemptive antiviral treatment.

HBV reactivation rates after HAIC for HCC (24\%-67\%) are reported to be higher than those after TACE possibly because of the higher dose of chemotherapeutic agents, as HAIC is carried out in shorter intervals. ${ }^{431,447,448}$ However, more research is needed to support the claim that HAIC has a higher reactivation rate than TACE, as only a few studies with a limited number of subjects have been reported and no comparative study with TACE has been performed.

Following the surgical resection of HCC, HBV reactivation with concomitant elevation in the HBV DNA level or abnormal biochemical liver function test is observed in 14\%-32\% of patients. ${ }^{449}$ In a prospective study comparing preemptive telbivudine administration to a nonadministered control group from the day of resection, the HBV reactivation rates were $2.5 \%$ and $31.8 \%$, respectively. While $57.1 \%$ of the control group showed HBV reactivation within 1 week following surgical resection, only $2.5 \%$ of the telbivudine-administered group showed reactivation within 4 weeks. ${ }^{439}$ The authors of that study recommend preemptive antiviral treatment before the surgical resection of HCC; however, that study involved only few patients at a single institution in China. Therefore, a large-scale multicenter study should be performed to determine a universal preemptive antiviral treatment before the surgical resection of HCC.

A study comparing preemptive lamivudine administration and a nonadministered control group following radiotherapy for HCC reports the HBV reactivation rates to be $0 \%$ and $21.8 \%$, respectively; meanwhile, alanine transaminase elevation occurred in $2.3 \%$ and $12.5 \%$ of patients, respectively. ${ }^{450}$ Another recent report suggests concurrent TACE and external radiotherapy may double the HBV reactivation rate compared to TACE alone. ${ }^{446}$ However, it is difficult to recommend preemptive antiviral treatment before external radiotherapy for HCC because of the lack of controlled prospective studies.

There are limited studies regarding HBV reactivation from
PEIT or RFA; nonetheless, the HBV reactivation rates for these therapies are reported to be $0 \%$ and 5.6\%-9.1\%, respectively. ${ }^{451,452}$ Meanwhile, no HBV reactivation was observed after sorafenib administration in a retrospective study. ${ }^{380}$ Regardless, more observations and research are required.

\section{HCV carriers}

Regarding HCV-related HCC, there are almost no reported cases of HCV reactivation or aggravation of hepatitis after HCC treatment. In a recent retrospective study on hepatitis virus reactivation comparing HCV- and HBV-related HCC after TACE, the rates of HCV and HBV reactivation, hepatitis, and liver failure were $26.5 \%$ and $32.5 \%, 10.2 \%$ and $34.8 \%$, and $0 \%$ and $10.9 \%$, respectively. ${ }^{453}$ No significant difference was observed between the HCV and HBV groups with respect to the reactivation rate, but the development of hepatitis and liver failure were significantly lower in the HCV-related HCC group. Hepatitis C treatments can be considered in patients with active chronic hepatitis C and completely eradicated HCC. As interferon and ribavirin administration may cause bone marrow suppression and transient aggravation of hepatitis, they are not recommended as preemptive treatments before cytotoxic chemotherapy in HCC patients.

\section{Recommendations (Table 2)}

1. Patients should be tested for hepatitis B surface antigen before starting cytotoxic chemotherapy or immunosuppressive therapy (A1).

2. Preemptive antiviral therapy is recommended for HBV carriers undergoing cytotoxic chemotherapy to prevent reactivation (A1). Preemptive antiviral therapy is considered for HBV infected patients receiving TACE (B1), hepatic arterial infusion chemotherapy (C1), surgical resection (C1), or EBRT (C1) to prevent reactivation.

3. Antiviral treatment for HBV reactivation should follow the recommendations of the current KASL guidelines (A1).

\section{DRUG TREATMENT FOR CANCER PAIN IN HCC}

Pain is one of the most troublesome symptoms in cancer patients. The prevalence of pain in cancer patients ranges from $45 \%$ to $53 \%,{ }^{45-456}$ and early aggressive palliative care including pain management could improve survival in lung cancer patients. ${ }^{457}$ A few studies have investigated the prevalence of pain in HCC patients, which is reported to range from $22 \%$ to $66.8 \%{ }^{455,458,459}$ Therefore, pain management should be considered an important aspect of palliative care for HCC patients. As most HCC patients have chronic liver disease and/or liver cirrhosis, their drug metabolism may be altered according to the degree of liver dysfunction. ${ }^{460}$ Furthermore, HCC patients receiving analgesics may suffer from more frequent and severe side effects. However, there is a paucity of studies on pain man- 
agement for patients with HCC and liver disease. ${ }^{461}$ Therefore, drug treatment for cancer pain in HCC patients should generally follow the principles of pain management for general solid tumors. ${ }^{462-464}$ However, drug selection, dosage, and administration interval might need to be adjusted according to the degree of liver function impairment.

The universal strategy for cancer pain treatment is based on a sequential three-step analgesics ladder approach from nonopioids to weak opioids and finally to strong opioids according to pain intensity and the efficacy of pain control. ${ }^{462-464}$ The main nonopioid analgesics such as acetaminophen and nonsteroidal anti-inflammatory drugs (NSAIDs) are indicated for the treatment of mild pain (numerical rating scale, 1-3). Meanwhile, weak opioids such as codeine, hydrocodone, and tramadol are indicated for mild to moderate pain (numerical rating scale, 4-6). Finally, strong opioids such as morphine, oxycodone, hydromorphone, fentanyl, and their analogues are the mainstay of analgesics for treating moderate to severe cancer-related pain (numerical rating scale, 7-10).

Acetaminophen is the most common cause of fulminant hepatic failure, ${ }^{465,466}$ but clinically significant hepatic injury is very rare when the dosage is limited to $4 \mathrm{~g} /$ day. ${ }^{467}$ Although one case report demonstrates that even therapeutic doses of acetaminophen less than $4 \mathrm{~g} /$ day in alcoholic patients without liver cirrhosis can result in acute liver failure, ${ }^{468}$ other studies show 4 $\mathrm{g} /$ day in alcoholic patients is not associated with a significant increase in liver toxicity. ${ }^{469,470}$ Moreover, one study shows a significant increase in the liver enzymes of alcoholic patients taking acetaminophen $4 \mathrm{~g} /$ day. $^{471}$ In patients with cirrhosis, acetaminophen 2-3 g/day is not associated with acute hepatic decompensation. ${ }^{472}$ Even though the half-life of oral acetaminophen is twice as long in patients with cirrhosis compared to healthy controls, ${ }^{473}$ significant hepatic injury is rare in patients with liver disease and/or cirrhosis at a dosage of less than $4 \mathrm{~g} /$ day. ${ }^{473,474}$ Nonetheless, most experts recommend lowering the dosage of acetaminophen to 2-3 g/day in patients with liver cirrhosis because of the inevitable possibility of altered drug metabolism and increased half-life. ${ }^{475,476}$

The unbound drug concentrations of NSAIDs are generally elevated in liver disease patients, which can lead to more severe side effects and toxicity. ${ }^{477}$ Indeed, roughly $10 \%$ of total druginduced hepatotoxicity cases are related to NSAIDs, ${ }^{478}$ and NSAID-induced liver injury is well documents. ${ }^{46,479}$ Moreover, NSAIDs can cause nephrotoxicity, ${ }^{480}$ gastric ulcer, hemorrhage, ${ }^{481,482}$ decompensation of liver function, etc. ${ }^{472}$

As the liver is the major site of metabolism for most opioids, impaired metabolism and excretion of opioids due to underlying liver disease in HCC patients can lead to increased side effects. Moreover, opioids are a well-known major precipitants of hepatic encephalopathy. ${ }^{474}$ Therefore, careful selection, and dosage and interval adjustment of drugs are required according to the liver metabolism of each opioid. ${ }^{476,483}$ Morphine is an active analgesic compound by itself, and more than 90\% of metabolites are excreted renally after glucuronidation in the liver. The half-life of morphine is approximately twice as long in cirrhotic patients as that in healthy controls. ${ }^{484,485}$ Furthermore, its bioavailability is 4-fold greater in patients with HCC (68\%) as that in healthy controls (17\%). ${ }^{486}$ As the analgesic effect of codeine is presumed to be secondary following its conversion to morphine, serum levels are not expected. The ceiling effect of codeine may cause side effects before achieving a sufficient analgesic effect. Similarly, hydrocodone is metabolized to hydromorphone before producing an analgesic effect, which results in variable serum levels. Meanwhile, tramadol has 10-fold less affinity for opioid receptors than codeine and exerts its analgesic effect via the peripheral pain pathway, which may result in fewer side effects in patients with liver disease. However, its elimination half-life is up to 3-fold greater in patients with primary liver carcinoma than that in controls. ${ }^{487}$ Oxycodone is converted to various metabolites including oxymorphone (an active metabolite), which may result in variable serum levels of metabolites and an unpredictable analgesic effect. The elimination half-life of oxycodone is prolonged, while its clearance is diminished with significant ventilation depression in pretransplantation liver cirrhosis patients compared to posttransplantation patients. ${ }^{488}$ Hydromorphone is an active analgesic compound by itself and is metabolized and excreted after glucuronidation. Liver dysfunction does not have a relatively substantial effect on hydromorphone; the half-life of hydromorphone does not differ significantly in patients with moderate hepatic impairment compared to controls. ${ }^{489}$ Although fentanyl is metabolized by cytochrome, its metabolism does not yield toxic metabolites, significantly alter serum levels in cirrhotic patients. ${ }^{490}$ Furthermore, it is not influenced by renal dysfunction. ${ }^{476,483}$

\section{Recommendations (Table 2)}

1. Careful consideration is required for pain management with medication in patients with HCC and underlying liver disease. The dosage and dosing intervals of analgesics should be determined on the basis of liver functions (C1).

2. In patients with HCC and chronic liver disease, the dosage of acetaminophen should be lowered (C1) and NSAIDs should be used with caution (B1).

3. In patients with HCC and chronic liver disease, opioid analgesics and their dosage should be selected carefully on the basis of drug metabolism and liver function (C1).

\section{ASSESSMENT OF TUMOR RESPONSE AND POSTTREAT- MENT FOLLOW-UP}

\section{Tumor response}

The main endpoint in cancer research is overall survival. Nonetheless, tumor response and time to progression are also considered pivotal for the surrogate assessment of efficacy. In 
oncology, tumor response was initially measured according to the 1979 World Health Organization (WHO) criteria as follows: ${ }^{491}$

(1) Complete response (CR): Complete disappearance of all known disease and no new lesions determined by two observations not less than 4 weeks apart.

(2) Partial response (PR): 50\% reduction in total tumor load of all measurable lesions determined by two observations not less than 4 weeks apart.

(3) Progressive disease (PD): 25\% increase in the size of one or more measurable lesions or the appearance of new lesions.

(4) Stable disease (SD): Cases not belonging to CR, PR, or PD.

However, several problems arose when applying these definitions to clinical practice. For example, there were discrepancies in the criteria for measuring tumor size among researchers. Furthermore, some researchers define progressive disease on the basis of the change in the size of one tumor, while others define it on the basis of the sum of the changes in the sizes of all tumors. Another limitation of the WHO criteria is properly reflecting the changes in tumor volume determined by recent advanced CT and MRI technologies. In order to overcome these problems, the Response Evaluation Criteria in Solid Tumors (RECIST) criteria and RECIST version 1.1 were developed and released in 2000 and 2009, respectively. In these criteria, overall responses are determined after evaluating the treatment responses for all lesions that are targeted or nontargeted. ${ }^{492,493}$ The criteria used to determine objective tumor responses for targeted lesions (or an index lesion) are as follows:

(1) Complete response (CR): Disappearance of all targeted lesions. Any pathologic lymph nodes must have reduction in short axis to $<10 \mathrm{~mm}$.

(2) Partial response (PR): At least a 30\% decrease in the sum of the diameters of target lesions with respect to baseline sum diameter.

(3) Progressive disease (PD): At least a 20\% increase in the sum of the diameter of the target lesion. In addition to the relative increase of $20 \%$, the sum must also demonstrate an absolute increase of at least $5 \mathrm{~mm}$.

(4) Stable disease: Cases not belonging to CR, PR, or PD.

Meanwhile, the criteria for the evaluation of nontargeted lesions are as follows: $:^{492,494}$

(1) Complete response: Disappearance of all nontargeted lesions and normalization of tumor marker levels. All lymph nodes must be nonpathologic in size (i.e., $<10 \mathrm{~mm}$ short axis).

(2) Noncomplete response/nonprogressive disease: Persistence of one or more on-target lesions and/or maintenance of tumor marker levels above the normal limits.

(3) Progressive disease: Progression of existing nontargeted lesions or appearance of new lesions.

However, these criteria were primarily designed to evaluate cytotoxic agents. Therefore, they do not address measures of antitumor activity besides tumor shrinkage; thus, the best response in these criteria might be stable disease. As acknowledged in the original RECIST publication, assessments based solely on changes in tumor size can be misleading when applied to other anticancer drugs such as molecular targeted therapies or other therapeutic interventions. ${ }^{492}$ Therefore, these determinations may be inaccurate. Several clinical studies on HCC demonstrate that the RECIST criteria do not mirror the extent of tumor necrosis induced by interventional therapies or new molecular targeted drugs. ${ }^{374,495}$ In theory, viable tumor formation should be assessed by CT or MRI studies, and tumor viability should be defined according to the uptake of contrast agent in the arterial phase of dynamic imaging studies. In fact, extensive tumor necrosis, which develops after local treatment, may not be paralleled by a decrease in lesion diameter. ${ }^{374,495}$ To overcome these limitations, the EASL developed new criteria for HCC treatment response that take into account the degree of necrosis. ${ }^{496}$ Furthermore, mRECIST criteria were first proposed by a panel of experts; ${ }^{497,498}$ this proposal is based on the fact that the diameter of the target lesions with viable tumors should guide all assessments. Specific modifications to the original criteria regarding the assessment of vascular invasion, lymph nodes, ascites, pleural effusion, and new lesions are summarized in Table 7. However, a limitation that should be noted is that the assessment of response to treatment based on the mRECIST criteria can be influenced by the image quality of CT/MRI as well as the subjective decisions of radiologists. Because there is no solid evidence indicating which set of criteria is superior, the panel of experts recommends determining whether a set of criteria outperforms the conventional RECIST criteria as well as correlations with pathologic studies and outcome prediction.

\section{Recommendations (Table 2)}

1. Assessment of response should follow both the RECIST and mRECIST criteria (B1).

\section{Follow-up after complete response}

Follow-up data after complete response in HCC are very limited. In cases of complete response after hepatic resection, transplantation, or percutaneous local ablation, follow-up intervals are determined on the basis of pretreatment risk factors and the treatment-specific risk of recurrence.

The 5-year recurrence rate following hepatic resection is up to $70 \%$ and is due to intrahepatic metastases and/or de novo carcinogenesis. ${ }^{96,423,499,500}$ Postoperative recurrence is usually classified as early (i.e., <2 years postoperatively) or late (i.e., $>2$ years postoperatively). ${ }^{142,497}$ Risk factors for recurrence are related to the tumor or underlying chronic liver disease. Tumor-related risk factors for recurrence are associated with early recurrence and include tumor size and number, degree of differentiation, vascular invasion, serum AFP (if elevated preoperatively), insufficient resection margin, and nonanatomical resection. ${ }^{142,146,499-502}$ Meanwhile, late recurrence risk factors are 
Table 7. Assessment of Tumor Response*

\begin{tabular}{|c|c|c|}
\hline & RECIST & mRECIST \\
\hline \multicolumn{3}{|l|}{ Target lesion response } \\
\hline $\mathrm{CR}$ & Disappearance of all target lesions & $\begin{array}{l}\text { Disappearance of any intratumor arterial enhancement } \\
\text { in all target lesions }\end{array}$ \\
\hline PR & $\begin{array}{l}\text { At least a } 30 \% \text { decrease in the sum of the diameters of } \\
\text { target lesions, taking the baseline sum of the diam- } \\
\text { eters of target lesions as a reference }\end{array}$ & $\begin{array}{l}\text { At least a } 30 \% \text { decrease in the sum of the diameters } \\
\text { of viable (enhancement in the arterial phase) target } \\
\text { lesions, taking the baseline sum of the diameters of } \\
\text { target lesions as a reference }\end{array}$ \\
\hline SD & Any case that does not qualify for either PR or PD & Any case that does not qualify for either PR or PD \\
\hline PD & $\begin{array}{l}\text { An increase of at least } 20 \% \text { in the sum of the diam- } \\
\text { eters of target lesions, taking the smallest sum of the } \\
\text { diameters of target lesions recorded since treatment } \\
\text { started as a reference }\end{array}$ & $\begin{array}{l}\text { An increase of at least } 20 \% \text { in the sum of the diam- } \\
\text { eters of viable (enhancing) target lesions, taking the } \\
\text { smallest sum of the diameters of viable (enhancing) } \\
\text { target lesions recorded since treatment started as } \\
\text { reference }\end{array}$ \\
\hline \multicolumn{3}{|l|}{ Nontarget lesions response } \\
\hline $\mathrm{CR}$ & Disappearance of all nontarget lesions & $\begin{array}{l}\text { Disappearance of any intratumor arterial enhancement } \\
\text { in all nontarget lesions }\end{array}$ \\
\hline $\mathrm{IR} / \mathrm{SD}$ & Persistence of one or more nontarget lesions & $\begin{array}{l}\text { Persistence of intratumor arterial enhancement in one } \\
\text { or more nontarget lesions }\end{array}$ \\
\hline PD & $\begin{array}{l}\text { Appearance of one or more new lesions and/or un- } \\
\text { equivocal progression of existing nontarget lesions }\end{array}$ & $\begin{array}{l}\text { Appearance of one or more new lesions and/or un- } \\
\text { equivocal progression of existing nontarget lesions }\end{array}$ \\
\hline \multicolumn{3}{|l|}{ mRECIST recommendations } \\
\hline Pleural effusion and ascites & \multicolumn{2}{|c|}{$\begin{array}{l}\text { Cytopathologic confirmation of the neoplastic nature of any effusion that appears or worsens during treatment is } \\
\text { required to declare PD }\end{array}$} \\
\hline Porta hepatis lymph node & \multicolumn{2}{|c|}{$\begin{array}{l}\text { Lymph nodes detected at the portal hepatitis can be considered malignant if the lymph node short axis is at leas } \\
2 \mathrm{~cm}\end{array}$} \\
\hline Portal vein invasion & \multicolumn{2}{|c|}{$\begin{array}{l}\text { Malignant portal vein invasion should be considered a nonmeasurable lesion and thus included in the nontarge } \\
\text { lesion group }\end{array}$} \\
\hline New lesion & \multicolumn{2}{|c|}{$\begin{array}{l}\text { A new lesion can be classified as HCC if its longest diameter is at least } 1 \mathrm{~cm} \text { and the enhancement pattern is typi- } \\
\text { cal of HCC. A lesion with an atypical radiological pattern can be diagnosed as HCC by evidence of at least 1-cm } \\
\text { interval growth }\end{array}$} \\
\hline \multicolumn{3}{|c|}{ Overall response assessment in mRECIST } \\
\hline Target lesion & Nontarget lesion & Overall response \\
\hline CR & CR & CR \\
\hline CR & $\mathrm{IR} / \mathrm{SD}$ & PR \\
\hline PR & Non-PD & PR \\
\hline SD & Non-PD & SD \\
\hline PD & Any & or no \\
\hline Any & Yes & or no \\
\hline Any & Any & PD \\
\hline
\end{tabular}

RECIST, Response Evaluation Criteria in Solid Tumors; mRECIST, modified RECIST; CR, complete response; PR, partial response; SD, stable disease; PD, progressive disease; IR, incomplete response; HCC, hepatocellular carcinoma.

${ }^{*}$ Adapted from J Hepatol 2012;56:908-943 ${ }^{58}$ and Semin Liver Dis 2010;30:52-60. ${ }^{498}$

related to underlying liver disease and include elevated serum HBV DNA during the perioperative period in cases of chronic hepatitis $B,{ }^{148,503-505}$ and persistent active inflammation and advanced degree of fibrosis in cases of chronic hepatitis $C^{506,507}$

The 5-year survival rate of liver transplantation exceeds 70\% and the recurrence rate is less than 15\% in patients meeting Mi- lan criteria. ${ }^{163}$ Major risk factors for recurrence after transplantation are tumor size and vascular invasion; other risk factors include tumor number, degree of differentiation, serum AFP level, and bilobar location of tumors. ${ }^{508-510}$ Recurrence occurs in more than 90\% of patients within 2 years, 35\% of which occur in the liver if the abovementioned risk factors are present. A recent 
multicenter study from Korea reports that the recurrence-free survival was significantly lower in living donor than DDLT. ${ }^{511}$ Thus, follow-up after LDLT should be emphasized, especially in countries where most liver transplants are cases are from living donors, such as Korea.

Local recurrence rates up to 2 years after treatment are higher after RFA (2\%-18\%) or PEIT (11\%-45\%) than after surgery, respectively. ${ }^{249-251,259,260}$ The 4-year cumulative recurrence rate after RFA for single tumors $<3 \mathrm{~cm}$ is $57 \%$. Meanwhile, the 3-/5-year cumulative recurrence rates after RFA or PEIT for $\leq 3$ tumors $<3$ $\mathrm{cm}$ in diameter are 57\%/72\% and 64\%/77\%, respectively. ${ }^{512,513}$ Incomplete tumor necrosis often occurs after PEIT, and the local recurrence rate is up to $43 \%$ for large tumors $>3 \mathrm{~cm} .{ }^{514}$ RFA for patients with a single nodular tumor $<2 \mathrm{~cm}$ results in a 5-year survival rate of $70 \% .{ }^{247}$ Improved survival is anticipated when complete response after retreatment with RFA is achieved for local recurrence after RFA. Thus, early detection of local recurrence after RFA is of the utmost importance. ${ }^{515}$

Recurrence usually develops within 2 years after potentially curative treatments. Because early detection of recurrence allows the possibility of reapplication of curative treatment modalities, posttreatment monitoring should be performed frequently enough to detect recurrence as early as possible. However, the ideal monitoring intervals and methods require further research. Therefore, we recommend follow-up with dynamic enhanced imaging (i.e., CT or MRI) or MRI with liver-specific contrast agent every 2-6 months for the first 2 years after curative treatment. After 2 years without recurrence, follow-up can be performed at less frequent intervals. In addition, the monitoring interval should be individualized on the basis of patient-specific risk factors according to tumor biology and underlying liver diseases. $^{516,517}$

\section{Recommendations (Table 2)}

1. Patients with complete response after treatment should be followed up with imaging studies (i.e., dynamic contrastenhanced CT/MRI or MRI with liver-specific contrast agents) and serum tumor markers every 2-6 months in the first 2 years; thereafter, patients should be followed by regular checkups at individualized intervals (B1).

\section{CONFLICTS OF INTEREST}

Conflicts of interests of the HCC-KPGRC members are summarized in Appendix 2.

\section{ACKNOWLEDGEMENTS}

Contributors: 2014 KLCSG-NCC Korea HCC Practice Guidelines Revision Committee (Appendix 1)

Joong-Won Park*, Joon Hyeok Lee ${ }^{\dagger}$, Kyung-Suk Suh ${ }^{\dagger}$, Jin Wook Chung ${ }^{\dagger}$, Jinsil Seong ${ }^{\dagger}$, June Sung Lee, Won Young Tak,
Si Hyun Bae, Jong Eun Yeon, Moon Seok Choi, Yoon Jun Kim, Young-Suk Lim, Ji Hoon Kim, Do Young Kim, Hwi Young Kim, Bo Hyun Kim, Ho Yeong Lim, Kyung Sik Kim, Seong Hoon Kim, Gi Hong Choi, Dong-Sik Kim, Jong Man Kim, Jai Young Cho, Hae Won Lee, Nam-Joon Yi, Jeong Min Lee, Young Hwan Koh, Hyun Beom Kim, Young Kon Kim, Min Woo Lee, JinYoung Choi, Seung Soo Lee, Ji Hoon Shin, Sung Bum Cho, Yun $\mathrm{Ku}$ Cho, Tae Hyun Kim, Mi-Sook Kim, Jin Hee Kim, Hee Chul Park, Chul Seung Kay, and Eui Kyu Chie $\left({ }^{*}\right.$ Chairman; ${ }^{\dagger}$ Head of subcommittee).

The authors thank Dr. Hwi Young Kim and Dr. Bo Hyun Kim for English editing works.

This Guideline development was supported by a grant from the National Cancer Center, Korea (number: 1311250).

This Guideline has been published jointly by invitation and consent in both the Korean Journal of Radiology and Gut and Liver.

\section{REFERENCES}

1. Guyatt GH, Oxman AD, Vist GE, et al. GRADE: an emerging consensus on rating quality of evidence and strength of recommendations. BMJ 2008;336:924-926.

2. Guyatt GH, Oxman AD, Kunz R, et al. What is "quality of evidence" and why is it important to clinicians? BMJ 2008;336:995998.

3. Guyatt GH, Oxman AD, Kunz R, et al. Going from evidence to recommendations. BMJ 2008;336:1049-1051.

4. Schünemann HJ, Oxman $\mathrm{AD}$, Brozek J, et al. Grading quality of evidence and strength of recommendations for diagnostic tests and strategies. BMJ 2008;336:1106-1110.

5. Brouwers MC, Kho ME, Browman GP, et al. Development of the AGREE II, part 1: performance, usefulness and areas for improvement. CMAJ 2010;182:1045-1052.

6. Brouwers MC, Kho ME, Browman GP, et al. Development of the AGREE II, part 2: assessment of validity of items and tools to support application. CMAJ 2010;182:E472-E478.

7. National Cancer Control Institute. Cancer facts and figures 2011 [Internet]. Goyang: National Cancer Center; 2011 [cited 2014 Mar 26]. Available from: http://ncc.re.kr/english/infor/kccr.jsp.

8. National Cancer Control Institute. Cancer facts and figures 2010 [Internet]. Goyang: National Cancer Center; 2011 [cited 2014 Mar 26]. Available from: http://ncc.re.kr/manage/manage03_033_list. jsp.

9. Korean Association for the Study of the Liver. White paper on liver diseases in Korea. Seoul: Korean Association for the Study of the Liver, 2013.

10. Korean Statistical Information Service. Statistical database 2012 [Internet]. Daejeon: Statistics Korea; c2014 [cited 2014 Mar 26]. Available from: http://kosis.kr/eng/statisticsList/ statisticsList_01List.jsp?vwcd=MT_ETITLE\&parentId=A.

11. Llovet JM, Bustamante J, Castells A, et al. Natural history of un- 
treated nonsurgical hepatocellular carcinoma: rationale for the design and evaluation of therapeutic trials. Hepatology 1999;29: 62-67.

12. The Korean Liver Cancer Study Group; Korean Central Cancer Registry. A nationwide random sample study 2010 [Internet]. Seoul: The Korean Liver Cancer Study Group; 2010 [cited 2014 Mar 26]. Available from: http://www.klcsg.or.kr/index.asp.

13. Kwak HW, Park JW, Nam BH, et al. Clinical outcomes of a cohort series of patients with hepatocellular carcinoma in a hepatitis B virus-endemic area. J Gastroenterol Hepatol 2014;29:820-829.

14. Forner A, Llovet JM, Bruix J. Hepatocellular carcinoma. Lancet 2012;379:1245-1255.

15. El-Serag HB. Hepatocellular carcinoma. N Engl J Med 2011;365: 1118-1127.

16. Welzel TM, Graubard BI, Zeuzem S, El-Serag HB, Davila JA, McGlynn KA. Metabolic syndrome increases the risk of primary liver cancer in the United States: a study in the SEER-Medicare database. Hepatology 2011;54:463-471.

17. Hainaut P, Boyle P. Curbing the liver cancer epidemic in Africa. Lancet 2008;371:367-368.

18. Ioannou GN, Splan MF, Weiss NS, McDonald GB, Beretta L, Lee SP. Incidence and predictors of hepatocellular carcinoma in patients with cirrhosis. Clin Gastroenterol Hepatol 2007;5:938-945. e4.

19. Sangiovanni A, Prati GM, Fasani P, et al. The natural history of compensated cirrhosis due to hepatitis C virus: a 17-year cohort study of 214 patients. Hepatology 2006;43:1303-1310.

20. Chang MH, You SL, Chen CJ, et al. Decreased incidence of hepatocellular carcinoma in hepatitis B vaccinees: a 20-year followup study. J Natl Cancer Inst 2009;101:1348-1355.

21. WHO Publication. Hepatitis B vaccines: WHO position paper-recommendations. Vaccine 2010;28:589-590.

22. Korean Association for the Study of the Liver. KASL clinical practice guidelines: management of chronic hepatitis B. Clin Mol Hepatol 2012;18:109-162.

23. Niederau C, Heintges T, Lange $S$, et al. Long-term follow-up of HBeAg-positive patients treated with interferon alfa for chronic hepatitis B. N Engl J Med 1996;334:1422-1427.

24. Liaw YF, Sung JJ, Chow WC, et al. Lamivudine for patients with chronic hepatitis B and advanced liver disease. N Engl J Med 2004:351:1521-1531.

25. Thiele M, Gluud LL, Dahl EK, Krag A. Antiviral therapy for prevention of hepatocellular carcinoma and mortality in chronic hepatitis B: systematic review and meta-analysis. BMJ Open 2013;3:e003265.

26. Hosaka T, Suzuki F, Kobayashi M, et al. Long-term entecavir treatment reduces hepatocellular carcinoma incidence in patients with hepatitis B virus infection. Hepatology 2013;58:98-107.

27. Nishiguchi S, Kuroki T, Nakatani S, et al. Randomised trial of effects of interferon-alpha on incidence of hepatocellular carcinoma in chronic active hepatitis C with cirrhosis. Lancet 1995; 346:1051-1055.
28. Valla DC, Chevallier M, Marcellin P, et al. Treatment of hepatitis C virus-related cirrhosis: a randomized, controlled trial of interferon alfa-2b versus no treatment. Hepatology 1999;29:18701875.

29. Singal AG, Volk ML, Jensen D, Di Bisceglie AM, Schoenfeld PS. A sustained viral response is associated with reduced liver-related morbidity and mortality in patients with hepatitis $\mathrm{C}$ virus. Clin Gastroenterol Hepatol 2010;8:280-288.e1.

30. Lok AS, Everhart JE, Wright EC, et al. Maintenance peginterferon therapy and other factors associated with hepatocellular carcinoma in patients with advanced hepatitis C. Gastroenterology 2011;140:840-849.

31. Di Bisceglie AM, Shiffman ML, Everson GT, et al. Prolonged therapy of advanced chronic hepatitis $\mathrm{C}$ with low-dose peginterferon. N Engl J Med 2008;359:2429-2441.

32. Bruix J, Poynard T, Colombo M, et al. Maintenance therapy with peginterferon alfa-2b does not prevent hepatocellular carcinoma in cirrhotic patients with chronic hepatitis C. Gastroenterology 2011;140:1990-1999.

33. Yin J, Li N, Han Y, et al. Effect of antiviral treatment with nucleotide/nucleoside analogs on postoperative prognosis of hepatitis B virus-related hepatocellular carcinoma: a two-stage longitudinal clinical study. J Clin Oncol 2013;31:3647-3655.

34. Chen LT, Chen MF, Li LA, et al. Long-term results of a randomized, observation-controlled, phase III trial of adjuvant interferon Alfa-2b in hepatocellular carcinoma after curative resection. Ann Surg 2012;255:8-17.

35. Wong JS, Wong GL, Tsoi KK, et al. Meta-analysis: the efficacy of anti-viral therapy in prevention of recurrence after curative treatment of chronic hepatitis B-related hepatocellular carcinoma. Aliment Pharmacol Ther 2011;33:1104-1112.

36. Miao RY, Zhao HT, Yang HY, et al. Postoperative adjuvant antiviral therapy for hepatitis $\mathrm{B} / \mathrm{C}$ virus-related hepatocellular carcinoma: a meta-analysis. World J Gastroenterol 2010;16:29312942.

37. Shiratori Y, Shiina S, Teratani T, et al. Interferon therapy after tumor ablation improves prognosis in patients with hepatocellular carcinoma associated with hepatitis C virus. Ann Intern Med 2003;138:299-306.

38. Mazzaferro V, Romito R, Schiavo M, et al. Prevention of hepatocellular carcinoma recurrence with alpha-interferon after liver resection in HCV cirrhosis. Hepatology 2006;44:1543-1554.

39. Marrero JA, Fontana RJ, Fu S, Conjeevaram HS, Su GL, Lok AS. Alcohol, tobacco and obesity are synergistic risk factors for hepatocellular carcinoma. J Hepatol 2005;42:218-224.

40. El-Serag HB, Tran T, Everhart JE. Diabetes increases the risk of chronic liver disease and hepatocellular carcinoma. Gastroenterology 2004;126:460-468.

41. Bravi F, Bosetti C, Tavani A, Gallus S, La Vecchia C. Coffee reduces risk for hepatocellular carcinoma: an updated metaanalysis. Clin Gastroenterol Hepatol 2013;11:1413-1421.e1.

42. Singal A, Volk ML, Waljee A, et al. Meta-analysis: surveillance 
with ultrasound for early-stage hepatocellular carcinoma in patients with cirrhosis. Aliment Pharmacol Ther 2009;30:37-47.

43. Andersson KL, Salomon JA, Goldie SJ, Chung RT. Cost effectiveness of alternative surveillance strategies for hepatocellular carcinoma in patients with cirrhosis. Clin Gastroenterol Hepatol 2008;6:1418-1424.

44. Zhang BH, Yang BH, Tang ZY. Randomized controlled trial of screening for hepatocellular carcinoma. J Cancer Res Clin Oncol 2004;130:417-422.

45. Kudo M, Izumi N, Kokudo N, et al. Management of hepatocellular carcinoma in Japan: consensus-based clinical practice guidelines proposed by the Japan Society of Hepatology (JSH) 2010 updated version. Dig Dis 2011;29:339-364.

46. Kim MJ, Bae KW, Seo PJ, et al. Optimal cut-off value of PIVKAII for diagnosis of hepatocellular carcinoma: using ROC curve. Korean J Hepatol 2006;12:404-411.

47. Yoon YJ, Han KH, Kim C, et al. Clinical efficacy of serum PIVKAII in the diagnosis and follow up after treatment of hepatocellular carcinoma. Korean J Hepatol 2002;8:465-471.

48. Omata M, Lesmana LA, Tateishi R, et al. Asian Pacific Association for the Study of the Liver consensus recommendations on hepatocellular carcinoma. Hepatol Int 2010;4:439-474.

49. Wong GL, Chan HL, Tse YK, et al. On-treatment alpha-fetoprotein is a specific tumor marker for hepatocellular carcinoma in patients with chronic hepatitis B receiving entecavir. Hepatology 2014;59:986-995.

50. Krinsky GA, Lee VS, Theise ND, et al. Transplantation for hepatocellular carcinoma and cirrhosis: sensitivity of magnetic resonance imaging. Liver Transpl 2002;8:1156-1164.

51. Zacherl J, Pokieser P, Wrba F, et al. Accuracy of multiphasic helical computed tomography and intraoperative sonography in patients undergoing orthotopic liver transplantation for hepatoma: what is the truth? Ann Surg 2002;235:528-532.

52. Kim SH, Choi BI, Lee JY, et al. Diagnostic accuracy of multi-/single-detector row CT and contrast-enhanced MRI in the detection of hepatocellular carcinomas meeting the Milan criteria before liver transplantation. Intervirology 2008;51 Suppl 1:52-60.

53. Makuuchi M, Kokudo N, Arii S, et al. Development of evidencebased clinical guidelines for the diagnosis and treatment of hepatocellular carcinoma in Japan. Hepatol Res 2008;38:37-51.

54. Colli A, Fraquelli M, Casazza G, et al. Accuracy of ultrasonography, spiral CT, magnetic resonance, and alpha-fetoprotein in diagnosing hepatocellular carcinoma: a systematic review. Am J Gastroenterol 2006;101:513-523.

55. Piscaglia F, Bolondi L. Recent advances in the diagnosis of hepatocellular carcinoma. Hepatol Res 2007;37 Suppl 2:S178-S192.

56. Lee MH, Kim SH, Park MJ, Park CK, Rhim H. Gadoxetic acidenhanced hepatobiliary phase MRI and high-b-value diffusionweighted imaging to distinguish well-differentiated hepatocellular carcinomas from benign nodules in patients with chronic liver disease. AJR Am J Roentgenol 2011;197:W868-W875.

57. Kim JE, Kim SH, Lee SJ, Rhim H. Hypervascular hepatocellular carcinoma $1 \mathrm{~cm}$ or smaller in patients with chronic liver disease: characterization with gadoxetic acid-enhanced MRI that includes diffusion-weighted imaging. AJR Am J Roentgenol 2011;196: W758-W765.

58. European Association for the Study of the Liver; European Organisation for Research and Treatment of Cancer. EASL-EORTC clinical practice guidelines: management of hepatocellular carcinoma. J Hepatol 2012;56:908-943.

59. Park JW, Kim JH, Kim SK, et al. A prospective evaluation of $18 \mathrm{~F}-$ FDG and 11C-acetate PET/CT for detection of primary and metastatic hepatocellular carcinoma. J Nucl Med 2008;49:1912-1921.

60. Kojiro M, Roskams T. Early hepatocellular carcinoma and dysplastic nodules. Semin Liver Dis 2005;25:133-142.

61. Matsui 0. Detection and characterization of hepatocellular carcinoma by imaging. Clin Gastroenterol Hepatol 2005;3(10 Suppl 2): S136-S140.

62. Korean Liver Cancer Study Group; National Cancer Center. Practice guidelines for management of hepatocellular carcinoma 2009. Korean J Hepatol 2009;15:391-423.

63. Roskams T, Kojiro M. Pathology of early hepatocellular carcinoma: conventional and molecular diagnosis. Semin Liver Dis 2010;30:17-25.

64. Forner A, Vilana R, Ayuso C, et al. Diagnosis of hepatic nodules $20 \mathrm{~mm}$ or smaller in cirrhosis: prospective validation of the noninvasive diagnostic criteria for hepatocellular carcinoma. Hepatology 2008;47:97-104.

65. Stigliano R, Marelli L, Yu D, Davies N, Patch D, Burroughs AK. Seeding following percutaneous diagnostic and therapeutic approaches for hepatocellular carcinoma: what is the risk and the outcome? Seeding risk for percutaneous approach of HCC. Cancer Treat Rev 2007;33:437-447.

66. Silva MA, Hegab B, Hyde C, Guo B, Buckels JA, Mirza DF. Needle track seeding following biopsy of liver lesions in the diagnosis of hepatocellular cancer: a systematic review and meta-analysis. Gut 2008;57:1592-1596.

67. Bae SY, Choi MS, Gwak GY, et al. Comparison of usefulness of clinical diagnostic criteria for hepatocellular carcinoma in a hepatitis B endemic area. Clin Mol Hepatol 2012;18:185-194.

68. Pierce DA, Preston DL. Radiation-related cancer risks at low doses among atomic bomb survivors. Radiat Res 2000;154:178-186.

69. Preston DL, Shimizu Y, Pierce DA, Suyama A, Mabuchi K. Studies of mortality of atomic bomb survivors. Report 13: solid cancer and noncancer disease mortality: 1950-1997. Radiat Res 2003; 160:381-407.

70. Sont WN, Zielinski JM, Ashmore JP, et al. First analysis of cancer incidence and occupational radiation exposure based on the National Dose Registry of Canada. Am J Epidemiol 2001;153:309318.

71. Cardis E, Vrijheid M, Blettner M, et al. Risk of cancer after low doses of ionising radiation: retrospective cohort study in 15 countries. BMJ 2005;331:77.

72. Gilbert ES. Invited commentary: studies of workers exposed to 
low doses of radiation. Am J Epidemiol 2001;153:319-322.

73. Upton AC; National Coluncil on Radiation Protection and Measurements Scientific Committee 1-6. The state of the art in the 1990's: NCRP report No. 136 on the scientific bases for linearity in the dose-response relationship for ionizing radiation. Health Phys 2003;85:15-22.

74. Huda W, Ogden KM, Khorasani MR. Converting dose-length product to effective dose at CT. Radiology 2008;248:995-1003.

75. National Research Council, Committee to Assess Health Risks from Exposure to Low Levels of Ionizing Radiation. Health risks from exposure to low levels of ionizing radiation: BEIR VII, phase 2. Washington, DC: National Academies Press, 2006.

76. The 2007 Recommendations of the International Commission on Radiological Protection. ICRP publication 103. Ann ICRP 2007;37:1-332.

77. D’Amico G, Garcia-Tsao G, Pagliaro L. Natural history and prognostic indicators of survival in cirrhosis: a systematic review of 118 studies. J Hepatol 2006;44:217-231.

78. Llovet JM, Bruix J. Systematic review of randomized trials for unresectable hepatocellular carcinoma: chemoembolization improves survival. Hepatology 2003;37:429-442.

79. Meier V, Ramadori G. Clinical staging of hepatocellular carcinoma. Dig Dis 2009;27:131-141.

80. Ueno S, Tanabe G, Nuruki K, et al. Prognostic performance of the new classification of primary liver cancer of Japan (4th edition) for patients with hepatocellular carcinoma: a validation analysis. Hepatol Res 2002;24:395-403.

81. Liver Cancer Study Group of Japan. General rules for the clinical and pathological study of primary liver cancer. 3rd English ed. Tokyo: Kanehara, 2010.

82. Koh DW, Park JW, Oh SE, et al. Characteristics of the patients with extrahepatic metastases developed in modified UICC T1-T2 stage hepatocellular carcinoma. Paper presented at: Asian Pacific Association for the Study of the Liver Annual Meeting; 2007; Kyoto, Japan. Abtract P-0573.

83. Bruix J, Sherman M. Management of hepatocellular carcinoma. Hepatology 2005;42:1208-1236.

84. Lang H, Sotiropoulos GC, Domland M, et al. Liver resection for hepatocellular carcinoma in non-cirrhotic liver without underlying viral hepatitis. Br J Surg 2005;92:198-202.

85. Capussotti L, Muratore A, Massucco P, Ferrero A, Polastri R, Bouzari H. Major liver resections for hepatocellular carcinoma on cirrhosis: early and long-term outcomes. Liver Transpl 2004;10(2 Suppl 1):S64-S68.

86. Poon RT, Fan ST, Lo CM, et al. Improving survival results after resection of hepatocellular carcinoma: a prospective study of 377 patients over 10 years. Ann Surg 2001;234:63-70.

87. Andreou A, Vauthey JN, Cherqui D, et al. Improved long-term survival after major resection for hepatocellular carcinoma: a multicenter analysis based on a new definition of major hepatectomy. J Gastrointest Surg 2013;17:66-77.

88. Kim JH, Choi DW, Kim SB. Saftey and long-term outcome following major hepatectomy for hepatocellular carcinoma combined with compensated liver cirrhosis. J Korean Surg Soc 2006;70:444-450.

89. Huang J, Zhang Y, Peng Z, et al. A modified TNM-7 staging system to better predict the survival in patients with hepatocellular carcinoma after hepatectomy. J Cancer Res Clin Oncol 2013;139:1709-1719.

90. Torzilli G, Belghiti J, Kokudo N, et al. A snapshot of the effective indications and results of surgery for hepatocellular carcinoma in tertiary referral centers: is it adherent to the EASL/AASLD recommendations? An observational study of the HCC East-West Study Group. Ann Surg 2013;257:929-937.

91. Pugh RN, Murray-Lyon IM, Dawson JL, Pietroni MC, Williams R. Transection of the oesophagus for bleeding oesophageal varices. Br J Surg 1973;60:646-649.

92. Farges O, Malassagne B, Flejou JF, Balzan S, Sauvanet A, Belghiti J. Risk of major liver resection in patients with underlying chronic liver disease: a reappraisal. Ann Surg 1999;229:210-215.

93. Belghiti J, Hiramatsu K, Benoist S, Massault P, Sauvanet A, Farges 0. Seven hundred forty-seven hepatectomies in the 1990s: an update to evaluate the actual risk of liver resection. J Am Coll Surg 2000;191:38-46.

94. Makuuchi M, Sano K. The surgical approach to HCC: our progress and results in Japan. Liver Transpl 2004;10(2 Suppl 1):S46-S52.

95. Fan ST, Lai EC, Lo CM, Ng IO, Wong J. Hospital mortality of major hepatectomy for hepatocellular carcinoma associated with cirrhosis. Arch Surg 1995;130:198-203.

96. Llovet JM, Fuster J, Bruix J. Intention-to-treat analysis of surgical treatment for early hepatocellular carcinoma: resection versus transplantation. Hepatology 1999;30:1434-1440.

97. An M, Park JW, Shin JA, et al. The adverse effect of indirectly diagnosed portal hypertension on the complications and prognosis after hepatic resection of hepatocellular carcinoma. Korean J Hepatol 2006;12:553-561.

98. Choi GH, Park JY, Hwang HK, et al. Predictive factors for longterm survival in patients with clinically significant portal hypertension following resection of hepatocellular carcinoma. Liver Int 2011;31:485-493.

99. Capussotti L, Ferrero A, Viganò L, Muratore A, Polastri R, Bouzari H. Portal hypertension: contraindication to liver surgery? World J Surg 2006;30:992-999.

100. Ishizawa T, Hasegawa K, Aoki T, et al. Neither multiple tumors nor portal hypertension are surgical contraindications for hepatocellular carcinoma. Gastroenterology 2008;134:1908-1916.

101. Cucchetti A, Ercolani G, Vivarelli M, et al. Is portal hypertension a contraindication to hepatic resection? Ann Surg 2009;250:922928.

102. Guglielmi A, Ruzzenente A, Conci S, Valdegamberi A, Iacono C. How much remnant is enough in liver resection? Dig Surg 2012;29:6-17.

103. Kim SU, Ahn SH, Park JY, et al. Prediction of postoperative hepatic insufficiency by liver stiffness measurement (FibroScan((R))) 
before curative resection of hepatocellular carcinoma: a pilot study. Hepatol Int 2008;2:471-477.

104. Wong JS, Wong GL, Chan AW, et al. Liver stiffness measurement by transient elastography as a predictor on posthepatectomy outcomes. Ann Surg 2013;257:922-928.

105. Cescon M, Colecchia A, Cucchetti A, et al. Value of transient elastography measured with FibroScan in predicting the outcome of hepatic resection for hepatocellular carcinoma. Ann Surg 2012;256:706-712.

106. Hammerstingl R, Huppertz A, Breuer J, et al. Diagnostic efficacy of gadoxetic acid (Primovist)-enhanced MRI and spiral CT for a therapeutic strategy: comparison with intraoperative and histopathologic findings in focal liver lesions. Eur Radiol 2008;18:457467.

107. Kim SH, Kim SH, Lee J, et al. Gadoxetic acid-enhanced MRI versus triple-phase MDCT for the preoperative detection of hepatocellular carcinoma. AJR Am J Roentgenol 2009;192:1675-1681.

108. Lin CY, Chen JH, Liang JA, Lin CC, Jeng LB, Kao CH. 18F-FDG PET or PET/CT for detecting extrahepatic metastases or recurrent hepatocellular carcinoma: a systematic review and meta-analysis. Eur J Radiol 2012;81:2417-2422.

109. Koneru B, Teperman LW, Manzarbeitia C, et al. A multicenter evaluation of utility of chest computed tomography and bone scans in liver transplant candidates with stages I and II hepatoma. Ann Surg 2005;241:622-628.

110. Kim IS, Lim YS, Yoon HK, et al. The effect of preoperative transarterial chemoembolization on the patient's outcome in resectable hepatocellular carcinoma. Korean J Med 2005;69:614-621.

111. Wu CC, Ho YZ, Ho WL, Wu TC, Liu TJ, P'eng FK. Preoperative transcatheter arterial chemoembolization for resectable large hepatocellular carcinoma: a reappraisal. Br J Surg 1995;82:122126.

112. Farges 0, Belghiti J, Kianmanesh R, et al. Portal vein embolization before right hepatectomy: prospective clinical trial. Ann Surg 2003;237:208-217.

113. Tanaka H, Hirohashi K, Kubo S, Shuto T, Higaki I, Kinoshita H. Preoperative portal vein embolization improves prognosis after right hepatectomy for hepatocellular carcinoma in patients with impaired hepatic function. Br J Surg 2000;87:879-882.

114. Liu L, Wang Z, Jiang S, et al. Perioperative allogenenic blood transfusion is associated with worse clinical outcomes for hepatocellular carcinoma: a meta-analysis. PLoS One 2013;8:e64261.

115. Tsujita E, Taketomi A, Kitagawa D, et al. Selective hepatic vascular exclusion for the hepatic resection of HCC. Hepatogastroenterology 2007;54:527-530.

116. Eguchi S, Kanematsu T, Arii S, et al. Comparison of the outcomes between an anatomical subsegmentectomy and a non-anatomical minor hepatectomy for single hepatocellular carcinomas based on a Japanese nationwide survey. Surgery 2008;143:469-475.

117. Tanaka K, Shimada H, Matsumoto C, et al. Anatomic versus limited nonanatomic resection for solitary hepatocellular carcinoma. Surgery 2008;143:607-615.
118. Shi M, Guo RP, Lin XJ, et al. Partial hepatectomy with wide versus narrow resection margin for solitary hepatocellular carcinoma: a prospective randomized trial. Ann Surg 2007;245:36-43.

119. Poon RT, Fan ST, Ng IO, Wong J. Significance of resection margin in hepatectomy for hepatocellular carcinoma: a critical reappraisal. Ann Surg 2000;231:544-551.

120. Suh KS. Systematic hepatectomy for small hepatocellular carcinoma in Korea. J Hepatobiliary Pancreat Surg 2005;12:365-370.

121. Wakai T, Shirai Y, Sakata J, et al. Anatomic resection independently improves long-term survival in patients with T1-T2 hepatocellular carcinoma. Ann Surg Oncol 2007;14:1356-1365.

122. Yin Z, Fan X, Ye H, Yin D, Wang J. Short- and long-term outcomes after laparoscopic and open hepatectomy for hepatocellular carcinoma: a global systematic review and meta-analysis. Ann Surg Oncol 2013;20:1203-1215.

123. Nguyen KT, Gamblin TC, Geller DA. World review of laparoscopic liver resection-2,804 patients. Ann Surg 2009;250:831-841.

124. Lai EC, Yang GP, Tang CN. Robot-assisted laparoscopic liver resection for hepatocellular carcinoma: short-term outcome. Am J Surg 2013;205:697-702.

125. Vauthey JN, Lauwers GY, Esnaola NF, et al. Simplified staging for hepatocellular carcinoma. J Clin Oncol 2002;20:1527-1536.

126. Pawlik TM, Poon RT, Abdalla EK, et al. Critical appraisal of the clinical and pathologic predictors of survival after resection of large hepatocellular carcinoma. Arch Surg 2005;140:450-457.

127. Lee SG, Hwang S, Jung JP, Lee YJ, Kim KH, Ahn CS. Outcome of patients with huge hepatocellular carcinoma after primary resection and treatment of recurrent lesions. Br J Surg 2007;94:320-326.

128. Nishikawa H, Kimura T, Kita R, Osaki Y. Treatment for hepatocellular carcinoma in elderly patients: a literature review. J Cancer 2013;4:635-643.

129. Iakova P, Awad SS, Timchenko NA. Aging reduces proliferative capacities of liver by switching pathways of C/EBPalpha growth arrest. Cell 2003;113:495-506.

130. Mullen JT, Ribero D, Reddy SK, et al. Hepatic insufficiency and mortality in 1,059 noncirrhotic patients undergoing major hepatectomy. J Am Coll Surg 2007;204:854-862.

131. Hai L, Yong-Hong P, Yong F, Ren-Feng L. One-stage liver resection for spontaneous rupture of hepatocellular carcinoma. World J Surg 2005;29:1316-1318.

132. Bae JH, Hong SW, Heo TG, Lee H. Characteristics and prognosis after resection for ruptured hepatocellular carcinoma. Korean J HBP Surg 2006;10:37-41.

133. Buczkowski AK, Kim PT, Ho SG, et al. Multidisciplinary management of ruptured hepatocellular carcinoma. J Gastrointest Surg 2006;10:379-386.

134. Zhu Q, Li J, Yan JJ, Huang L, Wu MC, Yan YQ. Predictors and clinical outcomes for spontaneous rupture of hepatocellular carcinoma. World J Gastroenterol 2012;18:7302-7307.

135. Pawlik TM, Poon RT, Abdalla EK, et al. Hepatectomy for hepatocellular carcinoma with major portal or hepatic vein invasion: results of a multicenter study. Surgery 2005;137:403-410. 
136. Roayaie S, Jibara G, Taouli B, Schwartz M. Resection of hepatocellular carcinoma with macroscopic vascular invasion. Ann Surg Oncol 2013;20:3754-3760.

137. Moon DB, Hwang S, Wang HJ, et al. Surgical outcomes of hepatocellular carcinoma with bile duct tumor thrombus: a Korean multicenter study. World J Surg 2013;37:443-451.

138. Belghiti J, Guevara OA, Noun R, Saldinger PF, Kianmanesh R. Liver hanging maneuver: a safe approach to right hepatectomy without liver mobilization. J Am Coll Surg 2001;193:109-111.

139. Liu CL, Fan ST, Cheung ST, Lo CM, Ng IO, Wong J. Anterior approach versus conventional approach right hepatic resection for large hepatocellular carcinoma: a prospective randomized controlled study. Ann Surg 2006;244:194-203.

140. Minagawa M, Makuuchi M, Takayama T, Kokudo N. Selection criteria for repeat hepatectomy in patients with recurrent hepatocellular carcinoma. Ann Surg 2003;238:703-710.

141. Finkelstein SD, Marsh W, Demetris AJ, et al. Microdissectionbased allelotyping discriminates de novo tumor from intrahepatic spread in hepatocellular carcinoma. Hepatology 2003;37:871879.

142. Imamura H, Matsuyama Y, Tanaka E, et al. Risk factors contributing to early and late phase intrahepatic recurrence of hepatocellular carcinoma after hepatectomy. J Hepatol 2003;38:200-207.

143. Zhou L, Rui JA, Wang SB, Chen SG, Qu Q. Prognostic factors of solitary large hepatocellular carcinoma: the importance of differentiation grade. Eur J Surg Oncol 2011;37:521-525.

144. Li SH, Guo ZX, Xiao CZ, et al. Risk factors for early and late intrahepatic recurrence in patients with single hepatocellular carcinoma without macrovascular invasion after curative resection. Asian Pac J Cancer Prev 2013;14:4759-4763.

145. Nathan H, Schulick RD, Choti MA, Pawlik TM. Predictors of survival after resection of early hepatocellular carcinoma. Ann Surg 2009;249:799-805.

146. Portolani N, Coniglio A, Ghidoni S, et al. Early and late recurrence after liver resection for hepatocellular carcinoma: prognostic and therapeutic implications. Ann Surg 2006;243:229-235.

147. Kaibori M, Ishizaki M, Matsui K, Kwon AH. Predictors of microvascular invasion before hepatectomy for hepatocellular carcinoma. J Surg Oncol 2010;102:462-468.

148. Wu JC, Huang YH, Chau GY, et al. Risk factors for early and late recurrence in hepatitis B-related hepatocellular carcinoma. J Hepatol 2009;51:890-897.

149. Kim do Y, Paik YH, Ahn SH, et al. PIVKA-II is a useful tumor marker for recurrent hepatocellular carcinoma after surgical resection. Oncology 2007;72 Suppl 1:52-57.

150. Choi GH, Kim DH, Kang CM, et al. Prognostic factors and optimal treatment strategy for intrahepatic nodular recurrence after curative resection of hepatocellular carcinoma. Ann Surg Oncol 2008;15:618-629.

151. Nagano Y, Shimada H, Ueda M, et al. Efficacy of repeat hepatic resection for recurrent hepatocellular carcinomas. ANZ J Surg 2009;79:729-733.
152. Huang ZY, Liang BY, Xiong M, et al. Long-term outcomes of repeat hepatic resection in patients with recurrent hepatocellular carcinoma and analysis of recurrent types and their prognosis: a single-center experience in China. Ann Surg Oncol 2012;19:2515-2525.

153. Chan AC, Chan SC, Chok KS, et al. Treatment strategy for recurrent hepatocellular carcinoma: salvage transplantation, repeated resection, or radiofrequency ablation? Liver Transpl 2013;19:411419.

154. Chan DL, Alzahrani NA, Morris DL, Chua TC. Systematic review of efficacy and outcomes of salvage liver transplantation after primary hepatic resection for hepatocellular carcinoma. J Gastroenterol Hepatol 2014;29:31-41.

155. Katyal S, Oliver JH 3rd, Peterson MS, Ferris JV, Carr BS, Baron RL. Extrahepatic metastases of hepatocellular carcinoma. Radiology 2000;216:698-703.

156. Kuo SW, Chang YL, Huang PM, et al. Prognostic factors for pulmonary metastasectomy in hepatocellular carcinoma. Ann Surg Oncol 2007;14:992-997.

157. Nakagawa T, Kamiyama T, Nakanishi K, et al. Pulmonary resection for metastases from hepatocellular carcinoma: factors influencing prognosis. J Thorac Cardiovasc Surg 2006;131:12481254.

158. Koneru B, Cassavilla A, Bowman J, Iwatsuki S, Starzl TE. Liver transplantation for malignant tumors. Gastroenterol Clin North Am 1988;17:177-193.

159. Iwatsuki S, Starzl TE, Sheahan DG, et al. Hepatic resection versus transplantation for hepatocellular carcinoma. Ann Surg 1991; 214:221-228.

160. Bismuth H, Chiche L, Adam R, Castaing D, Diamond T, Dennison A. Liver resection versus transplantation for hepatocellular carcinoma in cirrhotic patients. Ann Surg 1993;218:145-151.

161. Bismuth H, Majno PE, Adam R. Liver transplantation for hepatocellular carcinoma. Semin Liver Dis 1999;19:311-322.

162. Mazzaferro V, Regalia E, Doci R, et al. Liver transplantation for the treatment of small hepatocellular carcinomas in patients with cirrhosis. N Engl J Med 1996;334:693-699.

163. Mazzaferro V, Bhoori S, Sposito C, et al. Milan criteria in liver transplantation for hepatocellular carcinoma: an evidence-based analysis of 15 years of experience. Liver Transpl 2011;17 Suppl 2:S44-S57.

164. European Liver Transplant Registry (ELTR) [Internet]. Villejuif Cedex: European Liver Transplant Registry; 2014 [cited 2014 Feb 18]. Available from: http://www.eltr.org/.

165. Organ Procurement and Transplantation Network (OPTN) [Internet]. Richmond: Organ Procurement and Transplantation Network; 2014 [cited 2014 Feb 18]. Available from: http://optn. transplant.hrsa.gov/.

166. Germani G, Gurusamy K, Garcovich M, et al. Which matters most: number of tumors, size of the largest tumor, or total tumor volume? Liver Transpl 2011;17 Suppl 2:S58-S66.

167. Sugimachi K, Shirabe K, Taketomi A, et al. Prognostic signifi- 
cance of preoperative imaging in recipients of living donor liver transplantation for hepatocellular carcinoma. Transplantation 2011;91:570-574.

168. Lee JM, Trevisani F, Vilgrain V, Wald C. Imaging diagnosis and staging of hepatocellular carcinoma. Liver Transpl 2011;17 Suppl 2: S34-S43.

169. Yang SH, Suh KS, Lee HW, et al. The role of (18)F-FDG-PET imaging for the selection of liver transplantation candidates among hepatocellular carcinoma patients. Liver Transpl 2006;12:16551660.

170. Kornberg A, Freesmeyer M, Barthel E, et al. 18F-FDG-uptake of hepatocellular carcinoma on PET predicts microvascular tumor invasion in liver transplant patients. Am J Transplant 2009;9: 592-600.

171. Clavien PA, Lesurtel M, Bossuyt PM, et al. Recommendations for liver transplantation for hepatocellular carcinoma: an international consensus conference report. Lancet Oncol 2012;13:e11e22.

172. Freeman RB Jr, Wiesner RH, Harper A, et al. The new liver allocation system: moving toward evidence-based transplantation policy. Liver Transpl 2002;8:851-858.

173. Pomfret EA, Washburn K, Wald C, et al. Report of a national conference on liver allocation in patients with hepatocellular carcinoma in the United States. Liver Transpl 2010;16:262-278.

174. Korean Network for Organ Sharing (KONOS) [Internet]. Seoul: Korean Network for Organ Sharing; 2014 [cited 2014 Mar 18]. Available from: http://www.konos.go.kr/.

175. Hwang S, Lee SG, Joh JW, Suh KS, Kim DG. Liver transplantation for adult patients with hepatocellular carcinoma in Korea: comparison between cadaveric donor and living donor liver transplantations. Liver Transpl 2005;11:1265-1272.

176. Kim MS. Comparison between Status with CTP score and MELD score in allocation of deceased donor liver: Korean nationalbased survey. Paper presented at: 2013 Congress of Asian Society of Transplantation; 2013; Kyoto, Japan.

177. Kim MS. Research for modification of emergency status in deceased donor liver allocation: development of revised MELD score system. Cheongju: Korea Centers for Disease Control and Prevention, 2014.

178. Roayaie S, Frischer JS, Emre SH, et al. Long-term results with multimodal adjuvant therapy and liver transplantation for the treatment of hepatocellular carcinomas larger than 5 centimeters. Ann Surg 2002;235:533-539.

179. Llovet JM, Bruix J. Novel advancements in the management of hepatocellular carcinoma in 2008. J Hepatol 2008;48 Suppl 1:S20-S37.

180. Samuel D, Colombo M, El-Serag H, Sobesky R, Heaton N. Toward optimizing the indications for orthotopic liver transplantation in hepatocellular carcinoma. Liver Transpl 2011;17 Suppl 2:S6-S13.

181. Ioannou GN, Perkins JD, Carithers RL Jr. Liver transplantation for hepatocellular carcinoma: impact of the MELD allocation system and predictors of survival. Gastroenterology 2008;134:1342-
1351.

182. Volk ML, Vijan S, Marrero JA. A novel model measuring the harm of transplanting hepatocellular carcinoma exceeding Milan criteria. Am J Transplant 2008;8:839-846.

183. Yao FY, Ferrell L, Bass NM, et al. Liver transplantation for hepatocellular carcinoma: expansion of the tumor size limits does not adversely impact survival. Hepatology 2001;33:1394-1403.

184. Mazzaferro V, Llovet JM, Miceli R, et al. Predicting survival after liver transplantation in patients with hepatocellular carcinoma beyond the Milan criteria: a retrospective, exploratory analysis. Lancet Oncol 2009;10:35-43.

185. Vibert E, Azoulay D, Hoti E, et al. Progression of alphafetoprotein before liver transplantation for hepatocellular carcinoma in cirrhotic patients: a critical factor. Am J Transplant 2010;10:129137.

186. Toso C, Trotter J, Wei A, et al. Total tumor volume predicts risk of recurrence following liver transplantation in patients with hepatocellular carcinoma. Liver Transpl 2008;14:1107-1115.

187. Mazzaferro V, Battiston C, Perrone S, et al. Radiofrequency ablation of small hepatocellular carcinoma in cirrhotic patients awaiting liver transplantation: a prospective study. Ann Surg 2004;240:900-909.

188. Llovet JM, Mas X, Aponte JJ, et al. Cost effectiveness of adjuvant therapy for hepatocellular carcinoma during the waiting list for liver transplantation. Gut 2002;50:123-128.

189. Yao FY, Bass NM, Nikolai B, et al. Liver transplantation for hepatocellular carcinoma: analysis of survival according to the intention-to-treat principle and dropout from the waiting list. Liver Transpl 2002;8:873-883.

190. Yao FY, Kerlan RK Jr, Hirose R, et al. Excellent outcome following down-staging of hepatocellular carcinoma prior to liver transplantation: an intention-to-treat analysis. Hepatology 2008;48:819-827.

191. Decaens T, Roudot-Thoraval F, Bresson-Hadni S, et al. Impact of pretransplantation transarterial chemoembolization on survival and recurrence after liver transplantation for hepatocellular carcinoma. Liver Transpl 2005;11:767-775.

192. Porrett PM, Peterman H, Rosen M, et al. Lack of benefit of pretransplant locoregional hepatic therapy for hepatocellular cancer in the current MELD era. Liver Transpl 2006;12:665-673.

193. Lesurtel M, Müllhaupt B, Pestalozzi BC, Pfammatter T, Clavien PA. Transarterial chemoembolization as a bridge to liver transplantation for hepatocellular carcinoma: an evidence-based analysis. Am J Transplant 2006;6:2644-2650.

194. Pelletier SJ, Fu S, Thyagarajan V, et al. An intention-to-treat analysis of liver transplantation for hepatocellular carcinoma using organ procurement transplant network data. Liver Transpl 2009;15:859-868.

195. Cucchetti A, Cescon M, Bigonzi E, et al. Priority of candidates with hepatocellular carcinoma awaiting liver transplantation can be reduced after successful bridge therapy. Liver Transpl 2011;17:1344-1354. 
196. De Luna W, Sze DY, Ahmed A, et al. Transarterial chemoinfusion for hepatocellular carcinoma as downstaging therapy and a bridge toward liver transplantation. Am J Transplant 2009;9:1158-1168.

197. Freeman RB Jr, Steffick DE, Guidinger MK, Farmer DG, Berg CL, Merion RM. Liver and intestine transplantation in the United States, 1997-2006. Am J Transplant 2008;8(4 Pt 2):958-976.

198. O'Connor JK, Trotter J, Davis GL, Dempster J, Klintmalm GB, Goldstein RM. Long-term outcomes of stereotactic body radiation therapy in the treatment of hepatocellular cancer as a bridge to transplantation. Liver Transpl 2012;18:949-954.

199. Bush DA, Kayali Z, Grove R, Slater JD. The safety and efficacy of high-dose proton beam radiotherapy for hepatocellular carcinoma: a phase 2 prospective trial. Cancer 2011;117:3053-3059.

200. Katz AW, Chawla S, Qu Z, Kashyap R, Milano MT, Hezel AF. Stereotactic hypofractionated radiation therapy as a bridge to transplantation for hepatocellular carcinoma: clinical outcome and pathologic correlation. Int J Radiat Oncol Biol Phys 2012;83:895900.

201. Ravaioli M, Grazi GL, Piscaglia F, et al. Liver transplantation for hepatocellular carcinoma: results of down-staging in patients initially outside the Milan selection criteria. Am J Transplant 2008;8:2547-2557.

202. Chapman WC, Majella Doyle MB, Stuart JE, et al. Outcomes of neoadjuvant transarterial chemoembolization to downstage hepatocellular carcinoma before liver transplantation. Ann Surg 2008;248:617-625.

203. Yao FY, Hirose R, LaBerge JM, et al. A prospective study on downstaging of hepatocellular carcinoma prior to liver transplantation. Liver Transpl 2005;11:1505-1514.

204. Bhoori S, Sposito C, Germini A, Coppa J, Mazzaferro V. The challenges of liver transplantation for hepatocellular carcinoma on cirrhosis. Transpl Int 2010;23:712-722.

205. Lewandowski RJ, Kulik LM, Riaz A, et al. A comparative analysis of transarterial downstaging for hepatocellular carcinoma: chemoembolization versus radioembolization. Am J Transplant 2009;9:1920-1928.

206. Korean Organ Donation Agency (KODA) [Internet]. Seoul: Korean Organ Donation Agency; 2014 [cited 2014 May 12]. Available from: http://www.koda1458.kr/.

207. Hwang S, Lee SG, Belghiti J. Liver transplantation for HCC: its role: Eastern and Western perspectives. J Hepatobiliary Pancreat Sci 2010;17:443-448.

208. Grant RC, Sandhu L, Dixon PR, Greig PD, Grant DR, McGilvray ID. Living vs. deceased donor liver transplantation for hepatocellular carcinoma: a systematic review and meta-analysis. Clin Transplant 2013;27:140-147.

209. Kulik LM, Fisher RA, Rodrigo DR, et al. Outcomes of living and deceased donor liver transplant recipients with hepatocellular carcinoma: results of the A2ALL cohort. Am J Transplant 2012;12: 2997-3007.

210. Kulik L, Abecassis M. Living donor liver transplantation for he- patocellular carcinoma. Gastroenterology 2004;127(5 Suppl 1): S277-S282.

211. Sarasin FP, Majno PE, Llovet JM, Bruix J, Mentha G, Hadengue A. Living donor liver transplantation for early hepatocellular carcinoma: a life-expectancy and cost-effectiveness perspective. Hepatology 2001;33:1073-1079.

212. Lee SG, Hwang S, Moon DB, et al. Expanded indication criteria of living donor liver transplantation for hepatocellular carcinoma at one large-volume center. Liver Transpl 2008;14:935-945.

213. Choi HJ, Kim DG, Na GH, Han JH, Hong TH, You YK. Clinical outcome in patients with hepatocellular carcinoma after livingdonor liver transplantation. World J Gastroenterol 2013;19:47374744.

214. Suh KS, Cho EH, Lee HW, Shin WY, Yi NJ, Lee KU. Liver transplantation for hepatocellular carcinoma in patients who do not meet the Milan criteria. Dig Dis 2007;25:329-333.

215. Kwon CH, Kim DJ, Han YS, et al. HCC in living donor liver transplantation: can we expand the Milan criteria? Dig Dis 2007; 25:313-319.

216. Sugawara Y, Tamura S, Makuuchi M. Living donor liver transplantation for hepatocellular carcinoma: Tokyo University series. Dig Dis 2007;25:310-312.

217. Ito T, Takada Y, Ueda M, et al. Expansion of selection criteria for patients with hepatocellular carcinoma in living donor liver transplantation. Liver Transpl 2007;13:1637-1644.

218. Taketomi A, Sanefuji K, Soejima Y, et al. Impact of des-gammacarboxy prothrombin and tumor size on the recurrence of hepatocellular carcinoma after living donor liver transplantation. Transplantation 2009;87:531-537.

219. Todo S, Furukawa H, Tada M; Japanese Liver Transplantation Study Group. Extending indication: role of living donor liver transplantation for hepatocellular carcinoma. Liver Transpl 2007; 13(11 Suppl 2):S48-S54.

220. Siegler M, Simmerling MC, Siegler JH, Cronin DC 2nd. Recipient deaths during donor surgery: a new ethical problem in living donor liver transplantation (LDLT). Liver Transpl 2006;12:358-360.

221. Ghobrial RM, Freise CE, Trotter JF, et al. Donor morbidity after living donation for liver transplantation. Gastroenterology 2008;135:468-476.

222. Brown RS Jr. Live donors in liver transplantation. Gastroenterology 2008;134:1802-1813.

223. Yi NJ, Suh KS, Cho JY, et al. Three-quarters of right liver donors experienced postoperative complications. Liver Transpl 2007;13:797-806.

224. Kim KH, Jung DH, Park KM, et al. Comparison of open and laparoscopic live donor left lateral sectionectomy. Br J Surg 2011; 98:1302-1308.

225. Hwang S, Lee SG, Lee YJ, et al. Lessons learned from 1,000 living donor liver transplantations in a single center: how to make living donations safe. Liver Transpl 2006;12:920-927.

226. Kim SJ, Na GH, Choi HJ, Yoo YK, Kim DG. Surgical outcome of right liver donors in living donor liver transplantation: single- 
center experience with 500 cases. J Gastrointest Surg 2012;16: 1160-1170.

227. Kim SH, Kim YK. Improving outcomes of living-donor right hepatectomy. Br J Surg 2013;100:528-534.

228. Shin M, Song S, Kim JM, et al. Donor morbidity including biliary complications in living-donor liver transplantation: single-center analysis of 827 cases. Transplantation 2012;93:942-948.

229. Chan SC, Chan AC, Sharr WW, et al. Perpetuating proficiency in donor right hepatectomy for living donor liver transplantation. Asian J Surg 2014;37:65-72.

230. Poon RT, Fan ST, Lo CM, Liu CL, Wong J. Long-term survival and pattern of recurrence after resection of small hepatocellular carcinoma in patients with preserved liver function: implications for a strategy of salvage transplantation. Ann Surg 2002;235:373-382.

231. Fuks D, Dokmak S, Paradis V, Diouf M, Durand F, Belghiti J. Benefit of initial resection of hepatocellular carcinoma followed by transplantation in case of recurrence: an intention-to-treat analysis. Hepatology 2012;55:132-140.

232. Sapisochin G, Castells L, Dopazo C, et al. Single HCC in cirrhotic patients: liver resection or liver transplantation? Long-term outcome according to an intention-to-treat basis. Ann Surg Oncol 2013;20:1194-1202.

233. Cucchetti A, Vitale A, Del Gaudio M, et al. Harm and benefits of primary liver resection and salvage transplantation for hepatocellular carcinoma. Am J Transplant 2010;10:619-627.

234. Hu Z, Wang W, Li Z, Ye S, Zheng SS. Recipient outcomes of salvage liver transplantation versus primary liver transplantation: a systematic review and meta-analysis. Liver Transpl 2012;18: 1316-1323.

235. Liu F, Wei Y, Wang W, et al. Salvage liver transplantation for recurrent hepatocellular carcinoma within UCSF criteria after liver resection. PLoS One 2012;7:e48932.

236. Moon JI, Kwon CH, Joh JW, et al. Primary versus salvage living donor liver transplantation for patients with hepatocellular carcinoma: impact of microvascular invasion on survival. Transplant Proc 2012;44:487-493.

237. Hwang S, Lee SG, Moon DB, et al. Salvage living donor liver transplantation after prior liver resection for hepatocellular carcinoma. Liver Transpl 2007;13:741-746.

238. Kim BW, Park YK, Kim YB, Wang HJ, Kim MW. Salvage liver transplantation for recurrent hepatocellular carcinoma after liver resection: feasibility of the Milan criteria and operative risk. Transplant Proc 2008;40:3558-3561.

239. Kaido T, Mori A, Ogura Y, et al. Living donor liver transplantation for recurrent hepatocellular carcinoma after liver resection. Surgery 2012;151:55-60.

240. Wu L, Hu A, Tam N, et al. Salvage liver transplantation for patients with recurrent hepatocellular carcinoma after curative resection. PLoS One 2012;7:e41820.

241. Li HY, Wei YG, Yan LN, Li B. Salvage liver transplantation in the treatment of hepatocellular carcinoma: a meta-analysis. World J Gastroenterol 2012;18:2415-2422.
242. Schwartz M, Roayaie S, Llovet J. How should patients with hepatocellular carcinoma recurrence after liver transplantation be treated? J Hepatol 2005;43:584-589.

243. Zimmerman MA, Trotter JF, Wachs M, et al. Sirolimus-based immunosuppression following liver transplantation for hepatocellular carcinoma. Liver Transpl 2008;14:633-638.

244. Toso C, Meeberg GA, Bigam DL, et al. De novo sirolimus-based immunosuppression after liver transplantation for hepatocellular carcinoma: long-term outcomes and side effects. Transplantation 2007;83:1162-1168.

245. Liang W, Wang D, Ling X, et al. Sirolimus-based immunosuppression in liver transplantation for hepatocellular carcinoma: a meta-analysis. Liver Transpl 2012;18:62-69.

246. Sala M, Llovet JM, Vilana R, et al. Initial response to percutaneous ablation predicts survival in patients with hepatocellular carcinoma. Hepatology 2004;40:1352-1360.

247. Livraghi T, Meloni F, Di Stasi M, et al. Sustained complete response and complications rates after radiofrequency ablation of very early hepatocellular carcinoma in cirrhosis: is resection still the treatment of choice? Hepatology 2008;47:82-89.

248. Lencioni R, Cioni D, Crocetti L, et al. Early-stage hepatocellular carcinoma in patients with cirrhosis: long-term results of percutaneous image-guided radiofrequency ablation. Radiology 2005;234:961-967.

249. Lin SM, Lin CJ, Lin CC, Hsu CW, Chen YC. Radiofrequency ablation improves prognosis compared with ethanol injection for hepatocellular carcinoma $<$ or $=4 \mathrm{~cm}$. Gastroenterology 2004;127: 1714-1723.

250. Shiina S, Teratani T, Obi S, et al. A randomized controlled trial of radiofrequency ablation with ethanol injection for small hepatocellular carcinoma. Gastroenterology 2005;129:122-130.

251. Lencioni RA, Allgaier HP, Cioni D, et al. Small hepatocellular carcinoma in cirrhosis: randomized comparison of radio-frequency thermal ablation versus percutaneous ethanol injection. Radiology 2003;228:235-240.

252. Kim YS, Lim HK, Rhim H, et al. Ten-year outcomes of percutaneous radiofrequency ablation as first-line therapy of early hepatocellular carcinoma: analysis of prognostic factors. J Hepatol 2013;58:89-97.

253. Shiina S, Tateishi R, Arano T, et al. Radiofrequency ablation for hepatocellular carcinoma: 10-year outcome and prognostic factors. Am J Gastroenterol 2012;107:569-577.

254. de Baère $T$, Risse 0 , Kuoch V, et al. Adverse events during radiofrequency treatment of 582 hepatic tumors. AJR Am J Roentgenol 2003;181:695-700.

255. Rhim H, Yoon KH, Lee JM, et al. Major complications after radiofrequency thermal ablation of hepatic tumors: spectrum of imaging findings. Radiographics 2003;23:123-134.

256. Song I, Rhim H, Lim HK, Kim YS, Choi D. Percutaneous radiofrequency ablation of hepatocellular carcinoma abutting the diaphragm and gastrointestinal tracts with the use of artificial ascites: safety and technical efficacy in 143 patients. Eur Radiol 
2009;19:2630-2640.

257. Lee MW, Rhim H, Cha DI, Kim YJ, Lim HK. Planning US for percutaneous radiofrequency ablation of small hepatocellular carcinomas (1-3 cm): value of fusion imaging with conventional US and CT/MR images. J Vasc Interv Radiol 2013;24:958-965.

258. Kudo M, Hatanaka K, Maekawa K. Newly developed novel ultrasound technique, defect reperfusion ultrasound imaging, using sonazoid in the management of hepatocellular carcinoma. Oncology 2010;78 Suppl 1:40-45.

259. Lin SM, Lin CJ, Lin CC, Hsu CW, Chen YC. Randomised controlled trial comparing percutaneous radiofrequency thermal ablation, percutaneous ethanol injection, and percutaneous acetic acid injection to treat hepatocellular carcinoma of $3 \mathrm{~cm}$ or less. Gut 2005;54:1151-1156.

260. Brunello F, Veltri A, Carucci P, et al. Radiofrequency ablation versus ethanol injection for early hepatocellular carcinoma: a randomized controlled trial. Scand J Gastroenterol 2008;43:727735.

261. Cho YK, Kim JK, Kim MY, Rhim H, Han JK. Systematic review of randomized trials for hepatocellular carcinoma treated with percutaneous ablation therapies. Hepatology 2009;49:453-459.

262. Chen MS, Li JQ, Zheng Y, et al. A prospective randomized trial comparing percutaneous local ablative therapy and partial hepatectomy for small hepatocellular carcinoma. Ann Surg 2006; 243:321-328.

263. Feng K, Yan J, Li X, et al. A randomized controlled trial of radiofrequency ablation and surgical resection in the treatment of small hepatocellular carcinoma. J Hepatol 2012;57:794-802.

264. Huang J, Yan L, Cheng Z, et al. A randomized trial comparing radiofrequency ablation and surgical resection for HCC conforming to the Milan criteria. Ann Surg 2010;252:903-912.

265. Qi X, Tang Y, An D, et al. Radiofrequency ablation versus hepatic resection for small hepatocellular carcinoma: a meta-analysis of randomized controlled trials. J Clin Gastroenterol 2014;48:450457.

266. Cho YK, Kim JK, Kim WT, Chung JW. Hepatic resection versus radiofrequency ablation for very early stage hepatocellular carcinoma: a Markov model analysis. Hepatology 2010;51:1284-1290.

267. Shibata T, Isoda H, Hirokawa Y, Arizono S, Shimada K, Togashi K. Small hepatocellular carcinoma: is radiofrequency ablation combined with transcatheter arterial chemoembolization more effective than radiofrequency ablation alone for treatment? Radiology 2009;252:905-913.

268. Morimoto M, Numata K, Kondou M, Nozaki A, Morita S, Tanaka K. Midterm outcomes in patients with intermediate-sized hepatocellular carcinoma: a randomized controlled trial for determining the efficacy of radiofrequency ablation combined with transcatheter arterial chemoembolization. Cancer 2010;116:5452-5460.

269. Lu Z, Wen F, Guo Q, Liang H, Mao X, Sun H. Radiofrequency ablation plus chemoembolization versus radiofrequency ablation alone for hepatocellular carcinoma: a meta-analysis of randomized-controlled trials. Eur J Gastroenterol Hepatol 2013;25:187-
194.

270. Ni JY, Liu SS, Xu LF, Sun HL, Chen YT. Meta-analysis of radiofrequency ablation in combination with transarterial chemoembolization for hepatocellular carcinoma. World J Gastroenterol 2013;19:3872-3882.

271. Ho CM, Lee PH, Shau WY, Ho MC, Wu YM, Hu RH. Survival in patients with recurrent hepatocellular carcinoma after primary hepatectomy: comparative effectiveness of treatment modalities. Surgery 2012;151:700-709.

272. Liang HH, Chen MS, Peng ZW, et al. Percutaneous radiofrequency ablation versus repeat hepatectomy for recurrent hepatocellular carcinoma: a retrospective study. Ann Surg Oncol 2008;15:34843493.

273. Chan AC, Poon RT, Cheung TT, et al. Survival analysis of re-resection versus radiofrequency ablation for intrahepatic recurrence after hepatectomy for hepatocellular carcinoma. World J Surg 2012;36:151-156.

274. Cha DI, Lee MW, Rhim H, Choi D, Kim YS, Lim HK. Therapeutic efficacy and safety of percutaneous ethanol injection with or without combined radiofrequency ablation for hepatocellular carcinomas in high risk locations. Korean J Radiol 2013;14:240-247.

275. Ishii H, Okada S, Nose H, et al. Local recurrence of hepatocellular carcinoma after percutaneous ethanol injection. Cancer 1996;77:1792-1796.

276. Vilana R, Bruix J, Bru C, Ayuso C, Sole M, Rodes J. Tumor size determines the efficacy of percutaneous ethanol injection for the treatment of small hepatocellular carcinoma. Hepatology 1992;16:353-357.

277. Livraghi T, Bolondi L, Lazzaroni S, et al. Percutaneous ethanol injection in the treatment of hepatocellular carcinoma in cirrhosis: a study on 207 patients. Cancer 1992;69:925-929.

278. Daniele B, De Sio I, Izzo F, et al. Hepatic resection and percutaneous ethanol injection as treatments of small hepatocellular carcinoma: a Cancer of the Liver Italian Program (CLIP 08) retrospective case-control study. J Clin Gastroenterol 2003;36:63-67.

279. Huang GT, Lee PH, Tsang YM, et al. Percutaneous ethanol injection versus surgical resection for the treatment of small hepatocellular carcinoma: a prospective study. Ann Surg 2005;242:3642.

280. Wright AS, Sampson LA, Warner TF, Mahvi DM, Lee FT Jr. Radiofrequency versus microwave ablation in a hepatic porcine model. Radiology 2005;236:132-139.

281. Lubner MG, Brace CL, Hinshaw JL, Lee FT Jr. Microwave tumor ablation: mechanism of action, clinical results, and devices. J Vasc Interv Radiol 2010;21(8 Suppl):S192-S203.

282. Sotiropoulos GC, Lang H, Frilling A, et al. Resectability of hepatocellular carcinoma: evaluation of 333 consecutive cases at a single hepatobiliary specialty center and systematic review of the literature. Hepatogastroenterology 2006;53:322-329.

283. Bargellini I, Florio F, Golfieri R, Grosso M, Lauretti DL, Cioni R. Trends in utilization of transarterial treatments for hepatocellular carcinoma: results of a survey by the italian society of interven- 
tional radiology. Cardiovasc Intervent Radiol 2014;37:438-444.

284. Park JW, Sherman M, Colombo M, et al. Observations of hepatocellular carcinoma (HCC) management patterns from the global HCC bridge study: first characterization of the full study population. J Clin Oncol 2012;30 Suppl:abstr 4033.

285. Gaba RC. Chemoembolization practice patterns and technical methods among interventional radiologists: results of an online survey. AJR Am J Roentgenol 2012;198:692-699.

286. Satake M, Uchida H, Arai Y, et al. Transcatheter arterial chemoembolization (TACE) with lipiodol to treat hepatocellular carcinoma: survey results from the TACE Study Group of Japan. Cardiovasc Intervent Radiol 2008;31:756-761.

287. Brown DB, Gould JE, Gervais DA, et al. Transcatheter therapy for hepatic malignancy: standardization of terminology and reporting criteria. J Vasc Interv Radiol 2009;20(7 Suppl):S425-S434.

288. Bruix J, Sala M, Llovet JM. Chemoembolization for hepatocellular carcinoma. Gastroenterology 2004;127(5 Suppl 1):S179-S188.

289. Matsui O, Kadoya M, Yoshikawa J, et al. Small hepatocellular carcinoma: treatment with subsegmental transcatheter arterial embolization. Radiology 1993;188:79-83.

290. Llovet JM, Real MI, Montaña X, et al. Arterial embolisation or chemoembolisation versus symptomatic treatment in patients with unresectable hepatocellular carcinoma: a randomised controlled trial. Lancet 2002;359:1734-1739.

291. Lo CM, Ngan H, Tso WK, et al. Randomized controlled trial of transarterial lipiodol chemoembolization for unresectable hepatocellular carcinoma. Hepatology 2002;35:1164-1171.

292. Takayasu K, Arii S, Ikai I, et al. Prospective cohort study of transarterial chemoembolization for unresectable hepatocellular carcinoma in 8510 patients. Gastroenterology 2006;131:461-469.

293. Ikeda M, Arai Y, Park SJ, et al. Prospective study of transcatheter arterial chemoembolization for unresectable hepatocellular carcinoma: an Asian cooperative study between Japan and Korea. J Vasc Interv Radiol 2013;24:490-500.

294. Shim JH, Park JW, Choi JI, Kim HB, Lee WJ, Kim CM. Does postembolization fever after chemoembolization have prognostic significance for survival in patients with unresectable hepatocellular carcinoma? J Vasc Interv Radiol 2009;20:209-216.

295. Chan AO, Yuen MF, Hui CK, Tso WK, Lai CL. A prospective study regarding the complications of transcatheter intraarterial lipiodol chemoembolization in patients with hepatocellular carcinoma. Cancer 2002;94:1747-1752.

296. Miyayama S, Matsui O, Yamashiro M, et al. Ultraselective transcatheter arterial chemoembolization with a 2-f tip microcatheter for small hepatocellular carcinomas: relationship between local tumor recurrence and visualization of the portal vein with iodized oil. J Vasc Interv Radiol 2007;18:365-376.

297. Lee HS, Kim KM, Yoon JH, et al. Therapeutic efficacy of transcatheter arterial chemoembolization as compared with hepatic resection in hepatocellular carcinoma patients with compensated liver function in a hepatitis B virus-endemic area: a prospective cohort study. J Clin Oncol 2002;20:4459-4465.
298. Bargellini I, Sacco R, Bozzi E, et al. Transarterial chemoembolization in very early and early-stage hepatocellular carcinoma patients excluded from curative treatment: a prospective cohort study. Eur J Radiol 2012;81:1173-1178.

299. Yang HJ, Lee JH, Lee DH, et al. Small single-nodule hepatocellular carcinoma: comparison of transarterial chemoembolization, radiofrequency ablation, and hepatic resection by using inverse probability weighting. Radiology 2014;271:909-918.

300. Chung GE, Lee JH, Kim HY, et al. Transarterial chemoembolization can be safely performed in patients with hepatocellular carcinoma invading the main portal vein and may improve the overall survival. Radiology 2011;258:627-634.

301. Georgiades CS, Hong K, D’Angelo M, Geschwind JF. Safety and efficacy of transarterial chemoembolization in patients with unresectable hepatocellular carcinoma and portal vein thrombosis. J Vasc Interv Radiol 2005;16:1653-1659.

302. Lee HS, Kim JS, Choi IJ, Chung JW, Park JH, Kim CY. The safety and efficacy of transcatheter arterial chemoembolization in the treatment of patients with hepatocellular carcinoma and main portal vein obstruction: a prospective controlled study. Cancer 1997;79:2087-2094.

303. Chung JW, Park JH, Han JK, Choi BI, Han MC. Hepatocellular carcinoma and portal vein invasion: results of treatment with transcatheter oily chemoembolization. AJR Am J Roentgenol 1995;165:315-321.

304. Luo J, Guo RP, Lai EC, et al. Transarterial chemoembolization for unresectable hepatocellular carcinoma with portal vein tumor thrombosis: a prospective comparative study. Ann Surg Oncol 2011;18:413-420.

305. Lammer J, Malagari K, Vogl T, et al. Prospective randomized study of doxorubicin-eluting-bead embolization in the treatment of hepatocellular carcinoma: results of the PRECISION V study. Cardiovasc Intervent Radiol 2010;33:41-52.

306. Varela M, Real MI, Burrel M, et al. Chemoembolization of hepatocellular carcinoma with drug eluting beads: efficacy and doxorubicin pharmacokinetics. J Hepatol 2007;46:474-481.

307. Sacco R, Bargellini I, Bertini M, et al. Conventional versus doxorubicin-eluting bead transarterial chemoembolization for hepatocellular carcinoma. J Vasc Interv Radiol 2011;22:1545-1552.

308. Burrel M, Reig M, Forner A, et al. Survival of patients with hepatocellular carcinoma treated by transarterial chemoembolisation (TACE) using Drug Eluting Beads: implications for clinical practice and trial design. J Hepatol 2012;56:1330-1335.

309. Malagari K, Pomoni M, Moschouris H, et al. Chemoembolization with doxorubicin-eluting beads for unresectable hepatocellular carcinoma: five-year survival analysis. Cardiovasc Intervent Radiol 2012;35:1119-1128.

310. Prajapati HJ, Dhanasekaran R, El-Rayes BF, et al. Safety and efficacy of doxorubicin drug-eluting bead transarterial chemoembolization in patients with advanced hepatocellular carcinoma. $\mathrm{J}$ Vasc Interv Radiol 2013;24:307-315.

311. Mazzaferro V, Sposito C, Bhoori S, et al. Yttrium-90 radioembo- 
lization for intermediate-advanced hepatocellular carcinoma: a phase 2 study. Hepatology 2013;57:1826-1837.

312. Salem R, Lewandowski RJ, Mulcahy MF, et al. Radioembolization for hepatocellular carcinoma using Yttrium-90 microspheres: a comprehensive report of long-term outcomes. Gastroenterology 2010;138:52-64.

313. Sangro B, Carpanese L, Cianni R, et al. Survival after yttrium-90 resin microsphere radioembolization of hepatocellular carcinoma across Barcelona clinic liver cancer stages: a European evaluation. Hepatology 2011;54:868-878.

314. Kim DY, Park BJ, Kim YH, et al. Radioembolization with Yttrium-90 resin microspheres in hepatocellular carcinoma: a multicenter prospective study. Am J Clin Oncol. Epub 2013 Sep 21. http://dx.doi.org/10.1097/C0C.0b013e3182a78dba.

315. Hawkins MA, Dawson LA. Radiation therapy for hepatocellular carcinoma: from palliation to cure. Cancer 2006;106:1653-1663.

316. Kim TH, Kim DY, Park JW, et al. Dose-volumetric parameters predicting radiation-induced hepatic toxicity in unresectable hepatocellular carcinoma patients treated with three-dimensional conformal radiotherapy. Int J Radiat Oncol Biol Phys 2007;67:225231.

317. Schefter TE, Kavanagh BD, Timmerman RD, Cardenes HR, Baron A, Gaspar LE. A phase I trial of stereotactic body radiation therapy (SBRT) for liver metastases. Int J Radiat Oncol Biol Phys 2005;62:1371-1378.

318. Pan CC, Kavanagh BD, Dawson LA, et al. Radiation-associated liver injury. Int J Radiat Oncol Biol Phys 2010;76(3 Suppl):S94S100.

319. Andolino DL, Johnson CS, Maluccio M, et al. Stereotactic body radiotherapy for primary hepatocellular carcinoma. Int J Radiat Oncol Biol Phys 2011;81:e447-e453.

320. Bujold A, Massey CA, Kim JJ, et al. Sequential phase I and II trials of stereotactic body radiotherapy for locally advanced hepatocellular carcinoma. J Clin Oncol 2013;31:1631-1639.

321. Honda Y, Kimura T, Aikata H, et al. Stereotactic body radiation therapy combined with transcatheter arterial chemoembolization for small hepatocellular carcinoma. J Gastroenterol Hepatol 2013;28:530-536.

322. Huang WY, Jen YM, Lee MS, et al. Stereotactic body radiation therapy in recurrent hepatocellular carcinoma. Int J Radiat Oncol Biol Phys 2012;84:355-361.

323. Kang JK, Kim MS, Cho CK, et al. Stereotactic body radiation therapy for inoperable hepatocellular carcinoma as a local salvage treatment after incomplete transarterial chemoembolization. Cancer 2012;118:5424-5431.

324. Sanuki N, Takeda A, Oku Y, et al. Stereotactic body radiotherapy for small hepatocellular carcinoma: a retrospective outcome analysis in 185 patients. Acta Oncol 2014;53:399-404.

325. Yoon SM, Lim YS, Park MJ, et al. Stereotactic body radiation therapy as an alternative treatment for small hepatocellular carcinoma. PLoS One 2013;8:e79854.

326. Fukumitsu N, Sugahara S, Nakayama H, et al. A prospective study of hypofractionated proton beam therapy for patients with hepatocellular carcinoma. Int J Radiat Oncol Biol Phys 2009;74:831-836.

327. Kawashima M, Furuse J, Nishio T, et al. Phase II study of radiotherapy employing proton beam for hepatocellular carcinoma. J Clin Oncol 2005;23:1839-1846.

328. Komatsu S, Fukumoto T, Demizu Y, et al. Clinical results and risk factors of proton and carbon ion therapy for hepatocellular carcinoma. Cancer 2011;117:4890-4904.

329. Nakayama H, Sugahara S, Tokita M, et al. Proton beam therapy for hepatocellular carcinoma: the University of Tsukuba experience. Cancer 2009;115:5499-5506.

330. Bae SH, Kim MS, Cho CK, et al. Feasibility and efficacy of stereotactic ablative radiotherapy for Barcelona Clinic Liver Cancer-C stage hepatocellular carcinoma. J Korean Med Sci 2013;28:213219.

331. Cho JY, Paik YH, Park HC, et al. The feasibility of combined transcatheter arterial chemoembolization and radiotherapy for advanced hepatocellular carcinoma. Liver Int 2014;34:795-801.

332. Eun HS, Kim MJ, Kim HJ, et al. The retrospective cohort study for survival rate in patients with advanced hepatocellular carcinoma receiving radiotherapy or palliative care. Korean J Hepatol 2011;17:189-198.

333. Katamura Y, Aikata H, Takaki S, et al. Intra-arterial 5-fluorouracil/interferon combination therapy for advanced hepatocellular carcinoma with or without three-dimensional conformal radiotherapy for portal vein tumor thrombosis. J Gastroenterol 2009;44:492-502.

334. Kim DY, Park W, Lim DH, et al. Three-dimensional conformal radiotherapy for portal vein thrombosis of hepatocellular carcinoma. Cancer 2005;103:2419-2426.

335. Lo CH, Huang WY, Lee MS, et al. Stereotactic ablative radiotherapy for unresectable hepatocellular carcinoma patients who failed or were unsuitable for transarterial chemoembolization. Eur J Gastroenterol Hepatol 2014;26:345-352.

336. McIntosh A, Hagspiel KD, Al-Osaimi AM, et al. Accelerated treatment using intensity-modulated radiation therapy plus concurrent capecitabine for unresectable hepatocellular carcinoma. Cancer 2009;115:5117-5125.

337. Shirai S, Sato M, Suwa K, et al. Feasibility and efficacy of single photon emission computed tomography-based three-dimensional conformal radiotherapy for hepatocellular carcinoma $8 \mathrm{~cm}$ or more with portal vein tumor thrombus in combination with transcatheter arterial chemoembolization. Int J Radiat Oncol Biol Phys 2010;76:1037-1044.

338. Skinner HD, Sharp HJ, Kaseb AO, et al. Radiation treatment outcomes for unresectable hepatocellular carcinoma. Acta Oncol 2011;50:1191-1198.

339. Sugahara S, Nakayama H, Fukuda K, et al. Proton-beam therapy for hepatocellular carcinoma associated with portal vein tumor thrombosis. Strahlenther Onkol 2009;185:782-788.

340. Tanaka Y, Nakazawa T, Komori S, et al. Radiotherapy for patients 
with unresectable advanced hepatocellular carcinoma with invasion to intrahepatic large vessels: efficacy and outcomes. J Gastroenterol Hepatol 2014;29:352-357.

341. Xi M, Zhang L, Zhao L, et al. Effectiveness of stereotactic body radiotherapy for hepatocellular carcinoma with portal vein and/or inferior vena cava tumor thrombosis. PLoS One 2013;8:e63864.

342. Yamada K, Izaki K, Sugimoto K, et al. Prospective trial of combined transcatheter arterial chemoembolization and three-dimensional conformal radiotherapy for portal vein tumor thrombus in patients with unresectable hepatocellular carcinoma. Int J Radiat Oncol Biol Phys 2003;57:113-119.

343. Yoon SM, Lim YS, Won HJ, et al. Radiotherapy plus transarterial chemoembolization for hepatocellular carcinoma invading the portal vein: long-term patient outcomes. Int J Radiat Oncol Biol Phys 2012;82:2004-2011.

344. Yu JI, Park HC, Lim do H, et al. Prognostic index for portal vein tumor thrombosis in patients with hepatocellular carcinoma treated with radiation therapy. J Korean Med Sci 2011;26:10141022.

345. Hsu HC, Chen TY, Chiu KW, et al. Three-dimensional conformal radiotherapy for the treatment of arteriovenous shunting in patients with hepatocellular carcinoma. Br J Radiol 2007;80:38-42.

346. Meng MB, Cui YL, Lu Y, et al. Transcatheter arterial chemoembolization in combination with radiotherapy for unresectable hepatocellular carcinoma: a systematic review and meta-analysis. Radiother Oncol 2009;92:184-194.

347. Oh D, Lim do H, Park HC, et al. Early three-dimensional conformal radiotherapy for patients with unresectable hepatocellular carcinoma after incomplete transcatheter arterial chemoembolization: a prospective evaluation of efficacy and toxicity. Am J Clin Oncol 2010;33:370-375.

348. Seong J, Lee IJ, Shim SJ, et al. A multicenter retrospective cohort study of practice patterns and clinical outcome on radiotherapy for hepatocellular carcinoma in Korea. Liver Int 2009;29:147152.

349. Tang QH, Li AJ, Yang GM, et al. Surgical resection versus conformal radiotherapy combined with TACE for resectable hepatocellular carcinoma with portal vein tumor thrombus: a comparative study. World J Surg 2013;37:1362-1370.

350. Koo JE, Kim JH, Lim YS, et al. Combination of transarterial chemoembolization and three-dimensional conformal radiotherapy for hepatocellular carcinoma with inferior vena cava tumor thrombus. Int J Radiat Oncol Biol Phys 2010;78:180-187.

351. Yu JI, Park HC, Lim do H, et al. Scheduled interval trans-catheter arterial chemoembolization followed by radiation therapy in patients with unresectable hepatocellular carcinoma. J Korean Med Sci 2012;27:736-743.

352. Han KH, Seong J, Kim JK, Ahn SH, Lee do Y, Chon CY. Pilot clinical trial of localized concurrent chemoradiation therapy for locally advanced hepatocellular carcinoma with portal vein thrombosis. Cancer 2008;113:995-1003.

353. Park MS, Kim SU, Park JY, et al. Combination treatment of localized concurrent chemoradiation therapy and transarterial chemoembolization in locally advanced hepatocellular carcinoma with intrahepatic metastasis. Cancer Chemother Pharmacol 2013;71:165-173.

354. Chuma M, Taguchi H, Yamamoto Y, et al. Efficacy of therapy for advanced hepatocellular carcinoma: intra-arterial 5-fluorouracil and subcutaneous interferon with image-guided radiation. J Gastroenterol Hepatol 2011;26:1123-1132.

355. Choi SB, Kim KS, Park YN, et al. The efficacy of hepatic resection after neoadjuvant transarterial chemoembolization (TACE) and radiation therapy in hepatocellular carcinoma greater than $5 \mathrm{~cm}$ in size. J Korean Med Sci 2009;24:242-247.

356. Kim TH, Kim DY, Park JW, et al. Three-dimensional conformal radiotherapy of unresectable hepatocellular carcinoma patients for whom transcatheter arterial chemoembolization was ineffective or unsuitable. Am J Clin Oncol 2006;29:568-575.

357. Park W, Lim DH, Paik SW, et al. Local radiotherapy for patients with unresectable hepatocellular carcinoma. Int J Radiat Oncol Biol Phys 2005;61:1143-1150.

358. Dawson LA, McGinn CJ, Lawrence TS. Conformal chemoradiation for primary and metastatic liver malignancies. Semin Surg Oncol 2003;21:249-255.

359. Cheng SH, Lin YM, Chuang VP, et al. A pilot study of three-dimensional conformal radiotherapy in unresectable hepatocellular carcinoma. J Gastroenterol Hepatol 1999;14:1025-1033.

360. Huang JF, Wang LY, Lin ZY, et al. Incidence and clinical outcome of icteric type hepatocellular carcinoma. J Gastroenterol Hepatol 2002;17:190-195.

361. Jang JW, Kay CS, You CR, et al. Simultaneous multitarget irradiation using helical tomotherapy for advanced hepatocellular carcinoma with multiple extrahepatic metastases. Int J Radiat Oncol Biol Phys 2009;74:412-418.

362. Park YJ, Lim do H, Paik SW, et al. Radiation therapy for abdominal lymph node metastasis from hepatocellular carcinoma. J Gastroenterol 2006;41:1099-1106.

363. Yamashita H, Nakagawa K, Shiraishi K, et al. Radiotherapy for lymph node metastases in patients with hepatocellular carcinoma: retrospective study. J Gastroenterol Hepatol 2007;22:523527.

364. Yoon SM, Kim JH, Choi EK, et al. Radioresponse of hepatocellular carcinoma-treatment of lymph node metastasis. Cancer Res Treat 2004;36:79-84.

365. Zeng ZC, Tang ZY, Fan J, et al. Consideration of role of radiotherapy for lymph node metastases in patients with HCC: retrospective analysis for prognostic factors from 125 patients. Int $\mathrm{J}$ Radiat Oncol Biol Phys 2005;63:1067-1076.

366. He J, Zeng ZC, Tang ZY, et al. Clinical features and prognostic factors in patients with bone metastases from hepatocellular carcinoma receiving external beam radiotherapy. Cancer 2009;115:2710-2720.

367. Kaizu T, Karasawa K, Tanaka Y, et al. Radiotherapy for osseous metastases from hepatocellular carcinoma: a retrospective study 
of 57 patients. Am J Gastroenterol 1998;93:2167-2171.

368. Murakami R, Baba Y, Furusawa M, et al. Short communication: the value of embolization therapy in painful osseous metastases from hepatocellular carcinomas: comparative study with radiation therapy. Br J Radiol 1996;69:1042-1044.

369. Seong J, Koom WS, Park HC. Radiotherapy for painful bone metastases from hepatocellular carcinoma. Liver Int 2005;25:261265.

370. Taki Y, Yamaoka Y, Takayasu T, et al. Bone metastases of hepatocellular carcinoma after liver resection. J Surg Oncol 1992;50:1218.

371. Choi HJ, Cho BC, Sohn JH, et al. Brain metastases from hepatocellular carcinoma: prognostic factors and outcome: brain metastasis from HCC. J Neurooncol 2009;91:307-313.

372. Nakamura N, Igaki H, Yamashita H, et al. A retrospective study of radiotherapy for spinal bone metastases from hepatocellular carcinoma (HCC). Jpn J Clin Oncol 2007;37:38-43.

373. Jiang W, Zeng ZC, Zhang JY, Fan J, Zeng MS, Zhou J. Palliative radiation therapy for pulmonary metastases from hepatocellular carcinoma. Clin Exp Metastasis 2012;29:197-205.

374. Llovet JM, Ricci S, Mazzaferro V, et al. Sorafenib in advanced hepatocellular carcinoma. N Engl J Med 2008;359:378-390.

375. Cheng AL, Kang YK, Chen Z, et al. Efficacy and safety of sorafenib in patients in the Asia-Pacific region with advanced hepatocellular carcinoma: a phase III randomised, double-blind, placebo-controlled trial. Lancet Oncol 2009;10:25-34.

376. Cheng AL, Kang YK, Lin DY, et al. Sunitinib versus sorafenib in advanced hepatocellular cancer: results of a randomized phase III trial. J Clin Oncol 2013;31:4067-4075.

377. Johnson PJ, Qin S, Park JW, et al. Brivanib versus sorafenib as first-line therapy in patients with unresectable, advanced hepatocellular carcinoma: results from the randomized phase III BRISKFL study. J Clin Oncol 2013;31:3517-3524.

378. Cainap C, Qin S, Huang WT, et al. Phase III trial of linifanib versus sorafenib in patients with advanced hepatocellular carcinoma (HCC). J Clin Oncol 2013;31(Suppl 4):abstr 249.

379. Furuse J, Ishii H, Nakachi K, Suzuki E, Shimizu S, Nakajima K. Phase I study of sorafenib in Japanese patients with hepatocellular carcinoma. Cancer Sci 2008;99:159-165.

380. Shim JH, Park JW, Choi JI, Park BJ, Kim CM. Practical efficacy of sorafenib monotherapy for advanced hepatocellular carcinoma patients in a Hepatitis B virus-endemic area. J Cancer Res Clin Oncol 2009;135:617-625.

381. Kim JE, Ryoo BY, Ryu MH, et al. Sorafenib for hepatocellular carcinoma according to Child-Pugh class of liver function. Cancer Chemother Pharmacol 2011;68:1285-1290.

382. Lencioni R, Kudo M, Ye SL, et al. GIDEON (Global Investigation of therapeutic DEcisions in hepatocellular carcinoma and Of its treatment with sorafeNib): second interim analysis. Int J Clin Pract 2014;68:609-617.

383. Kim HY, Park JW, Joo J, et al. Worse outcome of sorafenib therapy associated with ascites and Child-Pugh score in advanced hepatocellular carcinoma. J Gastroenterol Hepatol 2013;28:17561761.

384. Brose MS, Frenette CT, Keefe SM, Stein SM. Management of sorafenib-related adverse events: a clinician's perspective. Semin Oncol 2014;41 Suppl 2:S1-S16.

385. Park JW, Amarapurkar D, Chao Y, et al. Consensus recommendations and review by an International Expert Panel on Interventions in Hepatocellular Carcinoma (EPOIHCC). Liver Int 2013;33: 327-337.

386. Cheng AL, Amarapurkar D, Chao Y, et al. Re-evaluating transarterial chemoembolization for the treatment of hepatocellular carcinoma: consensus recommendations and review by an International Expert Panel. Liver Int 2014;34:174-183.

387. Kim HY, Park JW, Joo J, et al. Severity and timing of progression predict refractoriness to transarterial chemoembolization in hepatocellular carcinoma. J Gastroenterol Hepatol 2012;27:10511056.

388. Park JW, Koh YH, Kim HB, et al. Phase II study of concurrent transarterial chemoembolization and sorafenib in patients with unresectable hepatocellular carcinoma. J Hepatol 2012;56:13361342.

389. Lencioni R, Llovet JM, Han G, et al. Sorafenib or placebo in combination with transarterial chemoembolization (TACE) with doxorubicin-eluting beads (DEBDOX) for intermediate-stage hepatocellular carcinoma (HCC): phase II, randomized, double-blind SPACE trial. J Clin Oncol 2012;30(Suppl 4):abstr LBA154.

390. Brandi G, de Rosa F, Agostini V, et al. Metronomic capecitabine in advanced hepatocellular carcinoma patients: a phase II study. Oncologist 2013;18:1256-1257.

391. Mir O, Coriat R, Boudou-Rouquette P, et al. Gemcitabine and oxaliplatin as second-line treatment in patients with hepatocellular carcinoma pre-treated with sorafenib. Med Oncol 2012;29:27932799.

392. Lee JE, Bae SH, Choi JY, Yoon SK, You YK, Lee MA. Epirubicin, cisplatin, 5-FU combination chemotherapy in sorafenib-refractory metastatic hepatocellular carcinoma. World J Gastroenterol 2014;20:235-241.

393. Chlebowski RT, Brzechwa-Adjukiewicz A, Cowden A, Block JB, Tong M, Chan KK. Doxorubicin (75 mg/m2) for hepatocellular carcinoma: clinical and pharmacokinetic results. Cancer Treat Rep 1984;68:487-491.

394. Choi TK, Lee NW, Wong J. Chemotherapy for advanced hepatocellular carcinoma: adriamycin versus quadruple chemotherapy. Cancer 1984;53:401-405.

395. Sciarrino E, Simonetti RG, Le Moli S, Pagliaro L. Adriamycin treatment for hepatocellular carcinoma: experience with 109 patients. Cancer 1985;56:2751-2755.

396. Tetef M, Doroshow J, Akman S, et al. 5-Fluorouracil and highdose calcium leucovorin for hepatocellular carcinoma: a phase II trial. Cancer Invest 1995;13:460-463.

397. Yang TS, Lin YC, Chen JS, Wang HM, Wang CH. Phase II study of gemcitabine in patients with advanced hepatocellular carci- 
noma. Cancer 2000;89:750-756.

398. Guan Z, Wang Y, Maoleekoonpairoj S, et al. Prospective randomised phase II study of gemcitabine at standard or fixed dose rate schedule in unresectable hepatocellular carcinoma. Br J Cancer 2003;89:1865-1869.

399. Yen Y, Lim DW, Chung V, et al. Phase II study of oxaliplatin in patients with unresectable, metastatic, or recurrent hepatocellular cancer: a California Cancer Consortium Trial. Am J Clin Oncol 2008;31:317-322.

400. Patt YZ, Hassan MM, Aguayo A, et al. Oral capecitabine for the treatment of hepatocellular carcinoma, cholangiocarcinoma, and gallbladder carcinoma. Cancer 2004;101:578-586.

401. Boige V, Taïeb J, Hebbar M, et al. Irinotecan as first-line chemotherapy in patients with advanced hepatocellular carcinoma: a multicenter phase II study with dose adjustment according to baseline serum bilirubin level. Eur J Cancer 2006;42:456-459.

402. Yuen MF, Poon RT, Lai CL, et al. A randomized placebo-controlled study of long-acting octreotide for the treatment of advanced hepatocellular carcinoma. Hepatology 2002;36:687-691.

403. Barbare JC, Bouché 0, Bonnetain F, et al. Treatment of advanced hepatocellular carcinoma with long-acting octreotide: a phase III multicentre, randomised, double blind placebo-controlled study. Eur J Cancer 2009;45:1788-1797.

404. Llovet JM, Sala M, Castells L, et al. Randomized controlled trial of interferon treatment for advanced hepatocellular carcinoma. Hepatology 2000;31:54-58.

405. Barbare JC, Bouché 0, Bonnetain F, et al. Randomized controlled trial of tamoxifen in advanced hepatocellular carcinoma. J Clin Oncol 2005;23:4338-4346.

406. Yeo W, Mok TS, Zee B, et al. A randomized phase III study of doxorubicin versus cisplatin/interferon alpha-2b/doxorubicin/ fluorouracil (PIAF) combination chemotherapy for unresectable hepatocellular carcinoma. J Natl Cancer Inst 2005;97:1532-1538.

407. Qin S, Bai Y, Lim HY, et al. Randomized, multicenter, open-label study of oxaliplatin plus fluorouracil/leucovorin versus doxorubicin as palliative chemotherapy in patients with advanced hepatocellular carcinoma from Asia. J Clin Oncol 2013;31:3501-3508.

408. Lee J, Park JO, Kim WS, et al. Phase II study of doxorubicin and cisplatin in patients with metastatic hepatocellular carcinoma. Cancer Chemother Pharmacol 2004;54:385-390.

409. Shim JH, Park JW, Nam BH, Lee WJ, Kim CM. Efficacy of combination chemotherapy with capecitabine plus cisplatin in patients with unresectable hepatocellular carcinoma. Cancer Chemother Pharmacol 2009;63:459-467.

410. Lee JO, Lee KW, Oh DY, et al. Combination chemotherapy with capecitabine and cisplatin for patients with metastatic hepatocellular carcinoma. Ann Oncol 2009;20:1402-1407.

411. Keam B, Oh DY, Lee SH, et al. A phase II study of 5-fluorouracil and cisplatin systemic chemotherapy for inoperable hepatocellular carcinoma with alpha fetoprotein as a predictive and prognostic marker. Mol Med Rep 2008;1:415-422.

412. Louafi S, Boige V, Ducreux M, et al. Gemcitabine plus oxaliplatin
(GEMOX) in patients with advanced hepatocellular carcinoma (HCC): results of a phase II study. Cancer 2007;109:1384-1390.

413. Boige V, Raoul JL, Pignon JP, et al. Multicentre phase II trial of capecitabine plus oxaliplatin (XELOX) in patients with advanced hepatocellular carcinoma: FFCD 03-03 trial. Br J Cancer 2007; 97:862-867.

414. Thomas MB. Systemic therapy for hepatocellular carcinoma. Cancer J 2008;14:123-127.

415. Terashima T, Yamashita T, Arai K, et al. Feasibility and efficacy of hepatic arterial infusion chemotherapy for advanced hepatocellular carcinoma after sorafenib. Hepatol Res 2014;44:11791185.

416. Jeong SW, Jang JY, Lee JE, et al. The efficacy of hepatic arterial infusion chemotherapy as an alternative to sorafenib in advanced hepatocellular carcinoma. Asia Pac J Clin Oncol 2012;8:164-171.

417. Lim TY, Cheong JY, Cho SW, et al. Effect of low dose 5-fluorouracil and cisplatin intra-arterial infusion chemotherapy in advanced hepatocellular carcinoma with decompensated cirrhosis. Korean J Hepatol 2006;12:65-73.

418. Woo HY, Bae SH, Park JY, et al. A randomized comparative study of high-dose and low-dose hepatic arterial infusion chemotherapy for intractable, advanced hepatocellular carcinoma. Cancer Chemother Pharmacol 2010;65:373-382.

419. Hamada A, Yamakado K, Nakatsuka A, Takaki H, Akeboshi M, Takeda K. Hepatic arterial infusion chemotherapy with use of an implanted port system in patients with advanced hepatocellular carcinoma: prognostic factors. J Vasc Interv Radiol 2004;15:835841.

420. Ueshima K, Kudo M, Takita M, et al. Hepatic arterial infusion chemotherapy using low-dose 5-fluorouracil and cisplatin for advanced hepatocellular carcinoma. Oncology 2010;78 Suppl 1:148-153.

421. Takaki-Hamabe S, Yamasaki T, Saeki I, et al. Hepatic arterial infusion chemotherapy for advanced hepatocellular carcinoma: is the addition of subcutaneous interferon-alpha-2b beneficial? Hepatol Res 2009;39:223-230.

422. Yamashita T, Arai K, Sunagozaka H, et al. Randomized, phase II study comparing interferon combined with hepatic arterial infusion of fluorouracil plus cisplatin and fluorouracil alone in patients with advanced hepatocellular carcinoma. Oncology 2011;81:281-290.

423. Roayaie S, Blume IN, Thung SN, et al. A system of classifying microvascular invasion to predict outcome after resection in patients with hepatocellular carcinoma. Gastroenterology 2009;137:850855.

424. Belghiti J, Panis Y, Farges O, Benhamou JP, Fekete F. Intrahepatic recurrence after resection of hepatocellular carcinoma complicating cirrhosis. Ann Surg 1991;214:114-117.

425. Chen X, Zhang B, Yin X, Ren Z, Qiu S, Zhou J. Lipiodolized transarterial chemoembolization in hepatocellular carcinoma patients after curative resection. J Cancer Res Clin Oncol 2013;139:773781. 
426. Lau WY, Leung TW, Ho SK, et al. Adjuvant intra-arterial iodine131-labelled lipiodol for resectable hepatocellular carcinoma: a prospective randomised trial. Lancet 1999;353:797-801.

427. Boucher E, Corbinais S, Rolland Y, et al. Adjuvant intra-arterial injection of iodine-131-labeled lipiodol after resection of hepatocellular carcinoma. Hepatology 2003;38:1237-1241.

428. Takayama T, Sekine T, Makuuchi M, et al. Adoptive immunotherapy to lower postsurgical recurrence rates of hepatocellular carcinoma: a randomised trial. Lancet 2000;356:802-807.

429. Shi HY, Wang SN, Wang SC, Chuang SC, Chen CM, Lee KT. Preoperative transarterial chemoembolization and resection for hepatocellular carcinoma: a nationwide Taiwan database analysis of long-term outcome predictors. J Surg Oncol 2014;109:487493.

430. Yeo W, Lam KC, Zee B, et al. Hepatitis B reactivation in patients with hepatocellular carcinoma undergoing systemic chemotherapy. Ann Oncol 2004;15:1661-1666.

431. Nagamatsu H, Itano S, Nagaoka S, et al. Prophylactic lamivudine administration prevents exacerbation of liver damage in HBe antigen positive patients with hepatocellular carcinoma undergoing transhepatic arterial infusion chemotherapy. Am J Gastroenterol 2004;99:2369-2375.

432. Lalazar G, Rund D, Shouval D. Screening, prevention and treatment of viral hepatitis B reactivation in patients with haematological malignancies. Br J Haematol 2007;136:699-712.

433. Mindikoglu AL, Regev A, Schiff ER. Hepatitis B virus reactivation after cytotoxic chemotherapy: the disease and its prevention. Clin Gastroenterol Hepatol 2006;4:1076-1081.

434. Lau GK, He ML, Fong DY, et al. Preemptive use of lamivudine reduces hepatitis B exacerbation after allogeneic hematopoietic cell transplantation. Hepatology 2002;36:702-709.

435. Yeo W, Chan PK, Zhong S, et al. Frequency of hepatitis B virus reactivation in cancer patients undergoing cytotoxic chemotherapy: a prospective study of 626 patients with identification of risk factors. J Med Virol 2000;62:299-307.

436. Lok AS, McMahon BJ. Chronic hepatitis B. Hepatology 2007;45: 507-539.

437. Wu XY, Li X, Chen ZH, et al. An optimized antiviral modification strategy for prevention of hepatitis B reactivation in patients undergoing prophylactic lamivudine and chemotherapy: a pilot study. Tumour Biol 2013;34:909-918.

438. Cortelezzi A, Viganò M, Zilioli VR, et al. Adefovir added to lamivudine for hepatitis B recurrent infection in refractory Bcell chronic lymphocytic leukemia on prolonged therapy with Campath-1H. J Clin Virol 2006;35:467-469.

439. Huang L, Li J, Yan J, et al. Antiviral therapy decreases viral reactivation in patients with hepatitis B virus-related hepatocellular carcinoma undergoing hepatectomy: a randomized controlled trial. J Viral Hepat 2013;20:336-342.

440. Huang G, Lai EC, Lau WY, et al. Posthepatectomy HBV reactivation in hepatitis B-related hepatocellular carcinoma influences postoperative survival in patients with preoperative low HBV-
DNA levels. Ann Surg 2013;257:490-505.

441. Lao XM, Luo G, Ye LT, et al. Effects of antiviral therapy on hepatitis B virus reactivation and liver function after resection or chemoembolization for hepatocellular carcinoma. Liver Int 2013;33: 595-604.

442. Lao XM, Wang D, Shi M, et al. Changes in hepatitis B virus DNA levels and liver function after transcatheter arterial chemoembolization of hepatocellular carcinoma. Hepatol Res 2011;41:553563.

443. Firpi RJ, Nelson DR. Management of viral hepatitis in hematologic malignancies. Blood Rev 2008;22:117-126.

444. Jang JW, Choi JY, Bae SH, et al. A randomized controlled study of preemptive lamivudine in patients receiving transarterial chemo-lipiodolization. Hepatology 2006;43:233-240.

445. Park JW, Park KW, Cho SH, et al. Risk of hepatitis B exacerbation is low after transcatheter arterial chemoembolization therapy for patients with HBV-related hepatocellular carcinoma: report of a prospective study. Am J Gastroenterol 2005;100:2194-2200.

446. Jang JW, Kwon JH, You CR, et al. Risk of HBV reactivation according to viral status and treatment intensity in patients with hepatocellular carcinoma. Antivir Ther 2011;16:969-977.

447. Tamori A, Nishiguchi S, Tanaka M, et al. Lamivudine therapy for hepatitis $\mathrm{B}$ virus reactivation in a patient receiving intra-arterial chemotherapy for advanced hepatocellular carcinoma. Hepatol Res 2003;26:77-80.

448. Nagamatsu H, Kumashiro R, Itano S, Matsugaki S, Sata M. Investigation of associating factors in exacerbation of liver damage after chemotherapy in patients with HBV-related HCC. Hepatol Res 2003;26:293-301.

449. Kubo S, Nishiguchi S, Hamba H, et al. Reactivation of viral replication after liver resection in patients infected with hepatitis B virus. Ann Surg 2001;233:139-145.

450. Kim JH, Park JW, Kim TH, Koh DW, Lee WJ, Kim CM. Hepatitis B virus reactivation after three-dimensional conformal radiotherapy in patients with hepatitis B virus-related hepatocellular carcinoma. Int J Radiat Oncol Biol Phys 2007;69:813-819.

451. Dan JQ, Zhang YJ, Huang JT, et al. Hepatitis B virus reactivation after radiofrequency ablation or hepatic resection for HBV-related small hepatocellular carcinoma: a retrospective study. Eur J Surg Oncol 2013;39:865-872.

452. Yoshida H, Yoshida H, Goto E, et al. Safety and efficacy of lamivudine after radiofrequency ablation in patients with hepatitis B virus-related hepatocellular carcinoma. Hepatol Int 2008;2:89-94.

453. Sung PS, Bae SH, Jang JW, et al. Differences in the patterns and outcomes of enhanced viral replication between hepatitis $\mathrm{C}$ virus and hepatitis B virus in patients with hepatocellular carcinoma during transarterial chemolipiodolization. Korean J Hepatol 2011; 17:299-306.

454. Hong SH, Roh SY, Kim SY, et al. Change in cancer pain management in Korea between 2001 and 2006: results of two nationwide surveys. J Pain Symptom Manage 2011;41:93-103.

455. Kim JY, Jang WY, Hur MH, et al. Prevalence and management 
of pain by different age groups of Korean cancer patients. Am J Hosp Palliat Care 2013;30:393-398.

456. van den Beuken-van Everdingen MH, de Rijke JM, Kessels AG, Schouten HC, van Kleef M, Patijn J. Prevalence of pain in patients with cancer: a systematic review of the past 40 years. Ann Oncol 2007;18:1437-1449.

457. Temel JS, Greer JA, Muzikansky A, et al. Early palliative care for patients with metastatic non-small-cell lung cancer. N Engl J Med 2010;363:733-742.

458. Carr BI, Pujol L. Pain at presentation and survival in hepatocellular carcinoma. J Pain 2010;11:988-993.

459. Ryu E, Kim K, Cho MS, Kwon IG, Kim HS, Fu MR. Symptom clusters and quality of life in Korean patients with hepatocellular carcinoma. Cancer Nurs 2010;33:3-10.

460. Verbeeck RK. Pharmacokinetics and dosage adjustment in patients with hepatic dysfunction. Eur J Clin Pharmacol 2008;64:1147-1161.

461. Radner H, Ramiro S, Buchbinder R, Landewé RB, van der Heijde $\mathrm{D}$, Aletaha D. Pain management for inflammatory arthritis (rheumatoid arthritis, psoriatic arthritis, ankylosing spondylitis and other spondylarthritis) and gastrointestinal or liver comorbidity. Cochrane Database Syst Rev 2012;1:CD008951.

462. World Health Organization. Cancer pain relief. 2nd ed. Geneva: World Health Organization, 1996.

463. Ministry of Health \& Welfare. Cancer pain management guideline. 5th ed. Seoul: Ministry of Health \& Welfare, 2012.

464. National Comprehensive Cancer Network. NCCN clinical practice guideline in oncology: adult cancer pain. Vol. 1. Fort Washington: National Comprehensive Cancer Network, 2013.

465. Rossi S, Assis DN, Awsare M, et al. Use of over-the-counter analgesics in patients with chronic liver disease: physicians' recommendations. Drug Saf 2008;31:261-270.

466. Larson AM, Polson J, Fontana RJ, et al. Acetaminophen-induced acute liver failure: results of a United States multicenter, prospective study. Hepatology 2005;42:1364-1372.

467. U.S. Department of Health and Human Services; U.S. Food and Drug Administration. Drugs: acetaminophen information [Internet]. Silver Spring: U.S. Food and Drug Administration; 2013 [cited 2013 Jan 15]. Available from: http://www.fda.gov/Drugs/ DrugSafety/InformationbyDrugClass/ucm165107.htm.

468. Mofredj A, Cadranel JF, Darchy B, et al. Hepatotoxicity caused by therapeutic doses of paracetamol in alcoholics: report of 2 cases of fatal hepatitis in cirrhosis. Ann Med Interne (Paris) 1999;150: 507-511.

469. Dart RC, Bailey E. Does therapeutic use of acetaminophen cause acute liver failure? Pharmacotherapy 2007;27:1219-1230.

470. Kuffner EK, Green JL, Bogdan GM, et al. The effect of acetaminophen (four grams a day for three consecutive days) on hepatic tests in alcoholic patients: a multicenter randomized study. BMC Med 2007;5:13.

471. Heard K, Green JL, Bailey JE, Bogdan GM, Dart RC. A randomized trial to determine the change in alanine aminotransferase during 10 days of paracetamol (acetaminophen) administration in subjects who consume moderate amounts of alcohol. Aliment Pharmacol Ther 2007;26:283-290.

472. Khalid SK, Lane J, Navarro V, Garcia-Tsao G. Use of over-thecounter analgesics is not associated with acute decompensation in patients with cirrhosis. Clin Gastroenterol Hepatol 2009;7:994999.

473. Villeneuve JP, Raymond G, Bruneau J, Colpron L, Pomier-Layrargues G. Pharmacokinetics and metabolism of acetaminophen in normal, alcoholic and cirrhotic subjects. Gastroenterol Clin Biol 1983;7:898-902.

474. Hirschfield GM, Kumagi T, Heathcote EJ. Preventative hepatology: minimising symptoms and optimising care. Liver Int 2008;28: 922-934.

475. Benson GD, Koff RS, Tolman KG. The therapeutic use of acetaminophen in patients with liver disease. Am J Ther 2005;12:133141.

476. Chandok N, Watt KD. Pain management in the cirrhotic patient: the clinical challenge. Mayo Clin Proc 2010;85:451-458.

477. Williams RL, Upton RA, Cello JP, et al. Naproxen disposition in patients with alcoholic cirrhosis. Eur J Clin Pharmacol 1984;27: 291-296.

478. Bessone F. Non-steroidal anti-inflammatory drugs: what is the actual risk of liver damage? World J Gastroenterol 2010;16:56515661.

479. Riley TR 3rd, Smith JP. Ibuprofen-induced hepatotoxicity in patients with chronic hepatitis C: a case series. Am J Gastroenterol 1998;93:1563-1565.

480. Ackerman Z, Cominelli F, Reynolds TB. Effect of misoprostol on ibuprofen-induced renal dysfunction in patients with decompensated cirrhosis: results of a double-blind placebo-controlled parallel group study. Am J Gastroenterol 2002;97:2033-2039.

481. Castro-Fernández M1, Sánchez-Muñoz D, Galán-Jurado MV, et al. Influence of nonsteroidal antiinflammatory drugs in gastrointestinal bleeding due to gastroduodenal ulcers or erosions in patients with liver cirrhosis. Gastroenterol Hepatol 2006;29:1114

482. Lee YC, Chang CH, Lin JW, Chen HC, Lin MS, Lai MS. Non-steroidal anti-inflammatory drugs use and risk of upper gastrointestinal adverse events in cirrhotic patients. Liver Int 2012;32:859-866.

483. Smith HS. Opioid metabolism. Mayo Clin Proc 2009;84:613-624.

484. Hasselström J, Eriksson S, Persson A, Rane A, Svensson JO, Säwe $\mathrm{J}$. The metabolism and bioavailability of morphine in patients with severe liver cirrhosis. Br J Clin Pharmacol 1990;29:289-297.

485. Tegeder I, Lötsch J, Geisslinger G. Pharmacokinetics of opioids in liver disease. Clin Pharmacokinet 1999;37:17-40.

486. Kotb HI, El-Kady SA, Emara SE, Fouad EA, El-Kabsh MY. Pharmacokinetics of controlled release morphine (MST) in patients with liver carcinoma. Br J Anaesth 2005;94:95-99.

487. Kotb HI, Fouad IA, Fares KM, Mostafa MG, Abd El-Rahman AM. Pharmacokinetics of oral tramadol in patients with liver cancer. $\mathrm{J}$ Opioid Manag 2008;4:99-104. 
488. Tallgren M, Olkkola KT, Seppälä T, Höckerstedt K, Lindgren L. Pharmacokinetics and ventilatory effects of oxycodone before and after liver transplantation. Clin Pharmacol Ther 1997;61:655-661.

489. Durnin C, Hind ID, Ghani SP, Yates DB, Molz KH. Pharmacokinetics of oral immediate-release hydromorphone (Dilaudid IR) in subjects with moderate hepatic impairment. Proc West Pharmacol Soc 2001;44:83-84.

490. Haberer JP, Schoeffler P, Couderc E, Duvaldestin P. Fentanyl pharmacokinetics in anaesthetized patients with cirrhosis. Br J Anaesth 1982;54:1267-1270.

491. Miller AB, Hoogstraten B, Staquet M, Winkler A. Reporting results of cancer treatment. Cancer 1981;47:207-214.

492. Therasse P, Arbuck SG, Eisenhauer EA, et al. New guidelines to evaluate the response to treatment in solid tumors. European Organization for Research and Treatment of Cancer, National Cancer Institute of the United States, National Cancer Institute of Canada. J Natl Cancer Inst 2000;92:205-216.

493. Bogaerts J, Ford R, Sargent D, et al. Individual patient data analysis to assess modifications to the RECIST criteria. Eur J Cancer 2009;45:248-260.

494. Eisenhauer EA, Therasse P, Bogaerts J, et al. New response evaluation criteria in solid tumours: revised RECIST guideline (version 1.1). Eur J Cancer 2009;45:228-247.

495. Forner A, Ayuso C, Varela M, et al. Evaluation of tumor response after locoregional therapies in hepatocellular carcinoma: are response evaluation criteria in solid tumors reliable? Cancer 2009;115:616-623.

496. Bruix J, Sherman M, Llovet JM, et al. Clinical management of hepatocellular carcinoma: conclusions of the Barcelona-2000 EASL conference. European Association for the Study of the Liver. J Hepatol 2001;35:421-430.

497. Llovet JM, Di Bisceglie AM, Bruix J, et al. Design and endpoints of clinical trials in hepatocellular carcinoma. J Natl Cancer Inst 2008;100:698-711.

498. Lencioni R, Llovet JM. Modified RECIST (mRECIST) assessment for hepatocellular carcinoma. Semin Liver Dis 2010;30:52-60.

499. Okada S, Shimada K, Yamamoto J, et al. Predictive factors for postoperative recurrence of hepatocellular carcinoma. Gastroenterology 1994;106:1618-1624.

500. Shirabe K, Kanematsu T, Matsumata T, Adachi E, Akazawa K, Sugimachi K. Factors linked to early recurrence of small hepatocellular carcinoma after hepatectomy: univariate and multivariate analyses. Hepatology 1991;14:802-805.

501. Poon RT, Fan ST, Lo CM, Liu CL, Wong J. Intrahepatic recurrence after curative resection of hepatocellular carcinoma: long-term results of treatment and prognostic factors. Ann Surg 1999;229: 216-222.

502. Adachi E, Maeda T, Matsumata T, et al. Risk factors for intrahepatic recurrence in human small hepatocellular carcinoma. Gastroenterology 1995;108:768-775.

503. Kim BK, Park JY, Kim do Y, et al. Persistent hepatitis B viral replication affects recurrence of hepatocellular carcinoma after cura- tive resection. Liver Int 2008:28:393-401.

504. Ohkubo K, Kato Y, Ichikawa T, et al. Viral load is a significant prognostic factor for hepatitis B virus-associated hepatocellular carcinoma. Cancer 2002;94:2663-2668.

505. Hung IF, Poon RT, Lai CL, Fung J, Fan ST, Yuen MF. Recurrence of hepatitis B-related hepatocellular carcinoma is associated with high viral load at the time of resection. Am J Gastroenterol 2008;103:1663-1673.

506. Kubo S, Nishiguchi S, Hirohashi K, et al. Clinicopathological criteria for multicentricity of hepatocellular carcinoma and risk factors for such carcinogenesis. Jpn J Cancer Res 1998;89:419-426.

507. Kubo S, Yamamoto T, Ikebe T, et al. Relationship between multicentric occurrence of hepatocellular carcinoma and histology of noncancerous hepatic tissue in patients with chronic hepatitis C. Jpn J Cancer Res 1999;90:1076-1080.

508. Todo S, Furukawa H. Living donor liver transplantation for adult patients with hepatocellular carcinoma: experience in Japan. Ann Surg 2004;240:451-459.

509. Shetty K, Timmins K, Brensinger C, et al. Liver transplantation for hepatocellular carcinoma validation of present selection criteria in predicting outcome. Liver Transpl 2004;10:911-918.

510. Löhe F, Angele MK, Gerbes AL, Löhrs U, Jauch KW, Schauer RJ. Tumour size is an important predictor for the outcome after liver transplantation for hepatocellular carcinoma. Eur J Surg Oncol 2005;31:994-999.

511. Park MS, Lee KW, Suh SW, et al. Living-donor liver transplantation associated with higher incidence of hepatocellular carcinoma recurrence than deceased-donor liver transplantation. Transplantation 2014;97:71-77.

512. Pompili M, Saviano A, de Matthaeis N, et al. Long-term effectiveness of resection and radiofrequency ablation for single hepatocellular carcinoma $</=3 \mathrm{~cm}$ : results of a multicenter Italian survey. J Hepatol 2013;59:89-97.

513. Hasegawa K, Kokudo N, Makuuchi M, et al. Comparison of resection and ablation for hepatocellular carcinoma: a cohort study based on a Japanese nationwide survey. J Hepatol 2013;58:724729.

514. Khan KN, Yatsuhashi H, Yamasaki K, et al. Prospective analysis of risk factors for early intrahepatic recurrence of hepatocellular carcinoma following ethanol injection. J Hepatol 2000;32:269278.

515. Rossi S, Ravetta V, Rosa L, et al. Repeated radiofrequency ablation for management of patients with cirrhosis with small hepatocellular carcinomas: a long-term cohort study. Hepatology 2011;53:136147.

516. Yang JD, Kim WR, Park KW, et al. Model to estimate survival in ambulatory patients with hepatocellular carcinoma. Hepatology 2012;56:614-621.

517. Kim BH, Park JW, Nam BH, Kwak HW, Kim WR. Validation of a model to estimate survival in ambulatory patients with hepatocellular carcinoma: a single-centre cohort study. Liver Int 2014; 34:e317-e323. 
Appendix 1. 2014 KLCSG-NCC Korea Hepatocellular Carcinoma Practice Guideline Revision Committee

\begin{tabular}{|c|c|c|}
\hline Chairman & Joong-Won Park & National Cancer Center, Korea \\
\hline Head of Hepatology & Joon Hyeok Lee & Sungkyunkwan University School of Medicine \\
\hline \multirow[t]{11}{*}{ Hepatologist } & June Sung Lee & Inje University College of Medicine \\
\hline & Won Young Tak & Kyungpook National University College of Medicine \\
\hline & Si Hyun Bae & The Catholic University of Korea College of Medicine \\
\hline & Jong Eun Yeon & Korea University School of Medicine \\
\hline & Moon Seok Choi & Sungkyunkwan University School of Medicine \\
\hline & Yoon Jun Kim & Seoul National University College of Medicine \\
\hline & Young-Suk Lim & University of Ulsan College of Medicine \\
\hline & Ji Hoon Kim & Korea University School of Medicine \\
\hline & Do Young Kim & Yonsei University College of Medicine \\
\hline & Hwi Young Kim & Seoul National University College of Medicine \\
\hline & Bo Hyun Kim & National Cancer Center, Korea \\
\hline Oncologist & Ho Yeong Lim & Sungkyunkwan University School of Medicine \\
\hline Head of Surgery & Kyung-Suk Suh & Seoul National University College of Medicine \\
\hline Hepatobiliary and & Kyung Sik Kim & Yonsei University College of Medicine \\
\hline \multirow[t]{7}{*}{ Transplantation Surgeon } & Seong Hoon Kim & National Cancer Center, Korea \\
\hline & Gi Hong Choi & Yonsei University College of Medicine \\
\hline & Dong-Sik Kim & Korea University College of Medicine \\
\hline & Jong Man Kim & Sungkyunkwan University School of Medicine \\
\hline & Jai Young Cho & Seoul National University College of Medicine \\
\hline & Hae Won Lee & Seoul National University College of Medicine \\
\hline & Nam-Joon Yi & Seoul National University College of Medicine \\
\hline Head of Radiology & Jin Wook Chung & Seoul National University College of Medicine \\
\hline \multirow[t]{10}{*}{ Radiologist } & Jeong Min Lee & Seoul National University College of Medicine \\
\hline & Young Hwan Koh & National Cancer Center, Korea \\
\hline & Hyun Beom Kim & National Cancer Center, Korea \\
\hline & Young Kon Kim & Sungkyunkwan University School of Medicine \\
\hline & Min Woo Lee & Sungkyunkwan University School of Medicine \\
\hline & Jin-Young Choi & Yonsei University College of Medicine \\
\hline & Seung Soo Lee & University of Ulsan College of Medicine \\
\hline & Ji Hoon Shin & University of Ulsan College of Medicine \\
\hline & Sung Bum Cho & Korea University College of Medicine \\
\hline & Yun Ku Cho & Seoul Veterans Hospital \\
\hline Head of Radiation Oncology & Jinsil Seong & Yonsei University College of Medicine \\
\hline \multirow[t]{7}{*}{ Radiation Oncologist } & Tae Hyun Kim & National Cancer Center, Korea \\
\hline & Mi-Sook Kim & Korea Institute of Radiological \& Medical Sciences \\
\hline & Jin Hee Kim & Keimyung University School of Medicine \\
\hline & Hee Chul Park & Sungkyunkwan University School of Medicine \\
\hline & Chul Seung Kay & The Catholic University of Korea College of Medicine \\
\hline & Eui Kyu Chie & Seoul National University College of Medicine \\
\hline & Won Sup Yoon & Korea University College of Medicine \\
\hline
\end{tabular}


Appendix 2. Disclosure of Conflict of Interests in the Past 2 Years

\begin{tabular}{|c|c|}
\hline Joong-Won Park & $\begin{array}{l}\text { Research support or lecture/consultant fees or taking part in clinical trials for Taiho, Bayer, Pfizer, BMS, Eisai, Roche, } \\
\text { Exelixis, Kowa }\end{array}$ \\
\hline Joon Hyeok Lee & $\begin{array}{l}\text { Research support or lecture/consultant fees or taking part in clinical trials for BMS, Creagen, MSD, Greencross-cell, } \\
\text { Dong-A, Bayer, Novartis, Bukwang, Gilead }\end{array}$ \\
\hline June Sung Lee & Nothing to disclose \\
\hline Won Young Tak & Nothing to disclose \\
\hline Si Hyun Bae & Research support or lecture/consultant fees or taking part in clinical trials for Bayer, BMS, Gilead, GSK \\
\hline Jong Eun Yeon & Research support or taking part in clinical trials for Yuhan, Jeil \\
\hline Moon Seok Choi & Research support or lecture/consultant fees for Bayer \\
\hline Yoon Jun Kim & $\begin{array}{l}\text { Research support or lecture/consultant fees or taking part in clinical trials for Creagen, LG, Pfizer, Hanmi, Roche, } \\
\text { Bayer, Pharmaking, Gilead, MSD, BMS, Gambro, Samil, Yuhan, CJ, Bukwang, Handok }\end{array}$ \\
\hline Young-Suk Lim & Research support or lecture/consultant fees or taking part in clinical trials for Bayer, BMS, Gilead \\
\hline Ji Hoon Kim & Nothing to disclose \\
\hline Do Young Kim & Nothing to disclose \\
\hline Hwi Young Kim & Nothing to disclose \\
\hline Bo Hyun Kim & Nothing to disclose \\
\hline Ho Yeong Lim & Nothing to disclose \\
\hline Kyung-Suk Suh & $\begin{array}{l}\text { Research support or lecture/consultant fees or taking part in clinical trials for Astellas, SK Chem, Greencross, Novartis, } \\
\text { Chong Kun Dang, Roch }\end{array}$ \\
\hline Kyung Sik Kim & Research support or lecture/consultant fees for Kabi-Fresenius, Samyang \\
\hline Seong Hoon Kim & Nothing to disclose \\
\hline Gi Hong Choi & Nothing to disclose \\
\hline Dong-Sik Kim & $\begin{array}{l}\text { Research support or lecture/consultant fees or taking part in clinical trials for Astellas, Hanmi, Pharmbio Korea, SK } \\
\text { Chem, Dong-A }\end{array}$ \\
\hline Jong Man Kim & $\begin{array}{l}\text { Research support or lecture/consultant fees or taking part in clinical trials for Astellas, SK Chem, Greencross, Novartis, } \\
\text { Chong Kun Dang, Roch, Sanofi, Hyundai, Gilead }\end{array}$ \\
\hline Jai Young Cho & Lecture for Coviden, Ethicon \\
\hline Hae Won Lee & Lecture for Chong Kun Dang \\
\hline Nam-Joon Yi & $\begin{array}{l}\text { Research support or lecture/consultant fees or taking part in clinical trials for Astellas, SK Chem, Greencross, Novartis, } \\
\text { Chong Kun Dang, Roch }\end{array}$ \\
\hline Jin Wook Chung & Research support or lecture/consultant fees or taking part in clinical trials for Biocompatibles, Guerbet \\
\hline Jeong Min Lee & $\begin{array}{l}\text { Research support or lecture/consultant fees or taking part in clinical trials for Bayer Primovist, Bayer, Guerbet, GE } \\
\text { Healthcare }\end{array}$ \\
\hline Young Hwan Koh & Nothing to disclose \\
\hline Hyun Beom Kim & Nothing to disclose \\
\hline Young Kon Kim & Lecture/consultant for Bayer Healthcare \\
\hline Min Woo Lee & Lecture/consultant for GE Healthcare, Bayer Healthcare \\
\hline Jin-Young Choi & Lecture/consultant for Bayer \\
\hline Seung Soo Lee & Nothing to disclose \\
\hline Ji Hoon Shin & Nothing to disclose \\
\hline Sung Bum Cho & Nothing to disclose \\
\hline Yun Ku Cho & Nothing to disclose \\
\hline Jinsil Seong & Nothing to disclose \\
\hline Tae Hyun Kim & Nothing to disclose \\
\hline Mi-Sook Kim & Nothing to disclose \\
\hline Jin Hee Kim & Nothing to disclose \\
\hline Hee Chul Park & Nothing to disclose \\
\hline Chul Seung Kay & Nothing to disclose \\
\hline Eui Kyu Chie & Lecture/consultant for Infinitt Healthcare, Merk-Sereno \\
\hline Won Sup Yoon & Nothing to disclose \\
\hline
\end{tabular}


Appendix 3. List of Clinical Questions

Internal medicine

1. Could the incidence of HCC be reduced by primary, secondary, or tertiary prevention?

2. How should we determine the high-risk group, imaging tests, roles of tumor markers, and diagnostic criteria?

3. Should we limit exposure to radiation to that necessary to diagnose and treat patients with HCC?

4. What staging system is applicable in Korea?

5. Who can obtain a survival benefit from sorafenib therapy? Is it safe and feasible in patients with hepatic impairment?

6. Does cytotoxic chemotherapy have a role in advanced HCC? Who should be considered for cytotoxic chemotherapy?

7. Does adjuvant therapy have a role after curative treatment for HCC?

8. When can preemptive antiviral therapy for hepatitis B be considered for patients undergoing treatment for HCC?

9. Can preemptive antiviral therapy for hepatitis $\mathrm{C}$ be considered for patients undergoing treatment for HCC?

10. How should we address pain management for patients with HCC?

11. For what kinds of pain medication should we adjust the dose and intervals? What can we adopt as a parameter of liver function in patients with HCC?

12. What criteria can we use to assess response to HCC treatment?

13. When and how should we follow-up patients after curative treatment for HCC?

\section{Surgery}

1. Should we consider size of the tumor and patient age when performing liver resection for HCC?

2. Is it applicable to perform the ICG-R15 test, Fibroscan, MRI, or PET to decide on liver resection?

3. How good are the outcomes of liver resection in patients with mild portal hypertension?

4. What is the safe volume of residual liver after liver resection in patients with liver cirrhosis?

5. Is anatomical resection superior to nonanatomical resection?

6. Is it useful to perform preconditioning when performing surgical resection for HCC?

7. Is it useful to perform surgical resection for HCC with bile duct invasion?

8. Is it useful to perform surgical resection for HCC with vascular invasion?

9. Is it useful to perform surgical resection for ruptured HCC?

10. What are the results (i.e., 5-year survival rate, mortality rate, and recurrence rate) of liver resection?

11. Is laparoscopic liver resection comparable to open resection? What are the advantages and indications?

12. When can we recommend liver transplantation as a primary treatment?

13. Do subcentimeter nodules alter the indications for liver transplantation?

14. What tests should be performed to investigate extrahepatic spread prior to liver transplantation?

15. How can we manage patients with HCC on the waiting list for liver transplantation? Is neoadjuvant therapy effective for them? Does neoadjuvant therapy decrease the withdrawal rate?

16. Can living donor liver transplantation be a substitute for deceased donor liver transplantation?

17. Do the Milan criteria have a role as indications for living donor liver transplantation or salvage transplantation?

18. Is the donor safe when performing living donor liver transplantation?

19. Can salvage transplantation be curative for recurred HCC?

20. Is it safe to perform salvage transplantation for recurred HCC after surgical resection?

21. What kinds of bridging therapies are available for patients on the transplant waiting list? Do they have a clinical impact?

22. When do we need to downstage HCC prior to liver transplantation? Can we improve clinical outcomes or broaden indications?

23. How should we treat liver transplant recipients with immunosuppressive or antiviral agents?

24. Should patients receive adjuvant therapy after liver transplantation? Is it useful or necessary? 
Appendix 3. Continued

Radiology

1. Is it applicable to diagnose HCC on the basis of noninvasive criteria using 4-phase multidetector CT or liver dynamic contrast-enhanced MRI? Should this only be done for patients with liver cirrhosis?

2. What is the size limit for a lesion for non-invasive diagnostic criteria?

3. What is the accuracy of noninvasive diagnostic criteria for a subcentimeter lesion with typical enhancement?

4. Can we use dynamic Gd-EOB-DTPA contrast-enhanced MRI as a surveillance test? Can it be allowed as a first-line test for a lesion identified by ultrasonography?

5. Can low signal intensity on the hepatobiliary phase of dynamic Gd-EOB-DTPA contrast-enhanced MRI be accepted as a parameter for noninvasive diagnostic criteria?

6. Should T2-weighted or diffusion-weighted imaging be included as a parameter for noninvasive diagnostic criteria?

7. Is contrast-enhanced ultrasound acceptable as a method for noninvasive diagnostic criteria?

8. How long should be the intervals between follow-up CT for patients with HCC?

9. Is radiofrequency ablation comparable with surgical resection for HCC with respect to survival?

10. When does combination therapy of radiofrequency ablation and transarterial chemoembolization have advantage over radiofrequency ablation monotherapy?

11. Are patients treated with radiofrequency ablation more likely to have microscopically residual disease (R1) than those treated with surgical resection?

12. Does local recurrence affect long-term survival after locoregional therapy?

13. How can the technical limitations of radiofrequency ablation due to location or invisibility of lesions be overcome?

14. Is percutaneous ethanol injection safer than radiofrequency ablation for HCC adjacent to the central bile duct?

15. Does radiofrequency ablation have a role for recurred HCC following surgical resection?

16. Do newer methods of locoregional therapy such as cryoablation or microwave ablation have a role?

17. When can we recommend transarterial chemoembolization as a first-line treatment?

18. Can we recommend transarterial chemoembolization as a curative therapy for HCCs that are curable but not amenable to other curative treatments?

19. Can we recommend transarterial chemoembolization for advanced HCCs with vascular invasion or metastasis?

20. Is there a role of chemolipiodolization?

21. Is there a role of combination therapy of transarterial chemoembolization and other therapies such as radiofrequency ablation, percutaneous ethanol injection, radiotherapy, and sorafenib?

22. When can we recommend drug-eluting bead transarterial chemoembolization? Does it have any advantage over conventional transarterial chemoembolization, or can we recommend it as a standard therapy?

23. Is transarterial radioembolization safe? When can we recommend transarterial radioembolization? Does transarterial radioembolization gain any advantage or survival benefit over conventional transarterial chemoembolization? Is it useful for downstaging prior to liver transplantation? Can we recommend it as a standard therapy?

Radio-oncology

1. When can external-beam radiotherapy be performed? What are the indications for external-beam radiotherapy?

2. Does combined radiotherapy play a role in the treatment of localized HCCs where transarterial chemoembolization is not expected to be effective?

3. Can we recommend external-beam radiotherapy for HCC with portal vein tumor thrombosis?

4. Can we recommend external-beam radiotherapy for HCCs $<5 \mathrm{~cm}$ not amenable to surgical resection or locoregional therapy?

5. Can we recommend external-beam radiotherapy to alleviate pain or symptoms caused by distant metastases?

6. Can external-beam radiotherapy play a role in bridging therapy in advanced HCCs prior to surgical resection?

7. Can we treat advanced HCCs with a combination of external-beam radiotherapy and systemic chemotherapy?

HCC, hepatocellular carcinoma; MRI, magnetic resonance imaging; PET, positron emission tomography; CT, computed tomography; Gd-EOBDTPA, gadolinium ethoxybenzyl diethylenetriamine pentaacetic acid. 
Appendix 4. Process of the Revision of 2014 KLCSG-NCC Korea Practice Guideline for the Management of Hepatocellular Carcinoma 2003 July: Release of 2003 KLCSG-NCC Korea practice guideline for the management of HCC 2009 June: Release of 2009 KLCSG-NCC Korea practice guideline for the management of HCC 2013 June: The KLCSG Chairman (SW Paik) suggested revision of KLCSG-NCC Korea guidelines 2014 September: Funding from National Cancer Center, Korea for the 2014 HCC practice guideline revision (grant \#1311250, P.I. JW Park) 2014 October: The KLCSG and NCC Korea approved the 2014 KLCSG-NCC Korea HCC Practice Guideline Revision Committee (PGRC) 2013 October: Opening and workshop of the HCC PGRC at Seoul National University Hospital 2013 November-2014 April: general meetings and communications between subcommittees 2014 April: Advisory board meeting

Advisory board members: Byung Ihn Choi (Seoul National University), Byung Chul Yoo (Sungkyunkwan University), Cheol Keun Park (Sungkyunkwan University), Kwang Hyub Han (Yonsei University), Hee Jung Wang (Ajou University), Yun Hwan Kim (Korea University), Kwan Sik Lee (Yonsei University), Seung Woon Paik (Sungkyunkwan University)

2014 May: Public hearing at Clinical Research Institution Auditorium, Seoul National University Hospital 2014 June: Both the KLCSG board of directors and NCC, Korea approved the final manuscript of HCC guidelines

2014 June 14: Release of 2014 KLCSG-NCC Korea Practice Guideline for the Management of HCC at the general meeting of KLCSG annual conference, Jeju province

KLCSG, Korean Liver Cancer Study Group; NCC, National Cancer Center; HCC, hepatocellular carcinoma.

Appendix 5. Liver Dynamic Contrast-Enhanced MRI Requirements for the Assessment of Hepatocellular Carcinoma

\begin{tabular}{|c|c|c|}
\hline Characteristic & Specification & Comment \\
\hline MRI equipment & $\geq 1.5 \mathrm{~T}$ & \\
\hline Contrast agent & $\begin{array}{l}\text { Gadolinium-based agents or hepatobiliary-specific } \\
\text { agents (gadolinium ethoxybenzyl diethylenetri- } \\
\text { amine pentaacetic acid) }\end{array}$ & $\begin{array}{l}\text { Necessary to inject the manufacturer's sug- } \\
\text { gested dose of contrast agent at a rapid rate of } \\
1-3 \mathrm{~mL} / \mathrm{sec}\end{array}$ \\
\hline Required imaging technique & $\begin{array}{l}\text { 1. T2-weighted image } \\
\text { 2. In/opposed phase T1-weighted image } \\
\text { 3. Diffusion-weighted image } \\
\text { 4. Fat-suppressed three-dimensional T1-weighted } \\
\text { image before and after contrast administration }\end{array}$ & $\begin{array}{l}\text { Necessary for the patient to hold their breath } \\
\text { for approximately } 20 \mathrm{sec} \text { for optimal dynamic } \\
\text { MRI }\end{array}$ \\
\hline $\begin{array}{l}\text { Dynamic phases and timing required for } \\
\text { dynamic contrast-enhanced MRI }\end{array}$ & $\begin{array}{l}\text { 1. Late arterial phase } \\
\text { 2. Portal venous phase } \\
\text { 3. Delayed or transitional* phase }\end{array}$ & $\begin{array}{l}\text { 1. } 5 \text { sec after maximum aortic enhancement } \\
\text { 2. } 1 \text { min after contrast injection (35-55 sec } \\
\text { after the arterial phase) } \\
\text { 3. 2-3 min after contrast injection (optimal } \\
\text { timing is } 3 \text { min) } \\
\text { * It is called the transitional phase because it is } \\
\text { enhanced by hepatocyte-specific uptake for } \\
\text { hepatobiliary-specific contrast agents. } \\
\text { Hepatobiliary-specific contrast agent is help- } \\
\text { ful for detecting small HCCs because it has a } \\
\text { hepatobiliary phase } 10-40 \text { min after contrast } \\
\text { injection and the liver parenchyma shows } \\
\text { strong enhancement by hepatocyte-specific } \\
\text { uptake }\end{array}$ \\
\hline
\end{tabular}

Slice thickness and resolution

Thickness: $<5$ to $\sim 8 \mathrm{~mm}$

Resolution: $<3 \mathrm{~mm}$

MRI, magnetic resonance imaging; HCC, hepatocellular carcinoma. 
Appendix 6. Liver Dynamic Contrast-Enhanced Computed Tomography Requirements for the Assessment of Hepatocellular Carcinoma

\begin{tabular}{lll}
\hline \multicolumn{1}{c}{ Characteristic } & \multicolumn{1}{c}{ Specification } & \multicolumn{1}{c}{ Comment } \\
\hline $\begin{array}{ll}\text { CT imaging equipment } \\
\text { Contrast agents }\end{array}$ & $\begin{array}{l}\text { Multidetector CT scanner with } \geq 4 \text { detectors } \\
\text { Contrast agents at a concentration of } \geq 300 \mathrm{mgI} / \mathrm{mL}\end{array}$ & $\begin{array}{c}\text { Necessary to rapidly inject contrast agent at a dose of } \\
520-600 \mathrm{mgI} / \mathrm{kg} \text { at } 2-5 \mathrm{~mL} / \mathrm{sec}\end{array}$ \\
Dynamic phases and timing & 1. Late arterial phase & $1.15-20 \mathrm{sec}$ after maximum aortic enhancement \\
required for dynamic & 2. Portal venous phase & 2. $60-80 \mathrm{sec}$ after contrast injection \\
contrast-enhanced CT & 3. Delayed phase & $3.2-3 \mathrm{~min}$ after contrast injection (optimal timing is 3 min) \\
Slice thickness/reconstruction & Thickness: $5 \mathrm{~mm}$ & \\
interval & Interval: $50 \%-100 \%$ of thickness & \\
\hline
\end{tabular}

CT, computed tomography. 